University of Louisville

ThinkIR: The University of Louisville's Institutional Repository

Electronic Theses and Dissertations

$12-2015$

\title{
Co-treatment with conjugated linoleic acid and nitrite modulates mitochondrial respiration and electron transport chain activity in vivo and attenuates mitochondrial dysfunction during cardiac injury.
}

Patrick Van Hoose

University of Louisville

Follow this and additional works at: https://ir.library.louisville.edu/etd

Part of the Biochemistry Commons, and the Molecular Biology Commons

\section{Recommended Citation}

Van Hoose, Patrick, "Co-treatment with conjugated linoleic acid and nitrite modulates mitochondrial respiration and electron transport chain activity in vivo and attenuates mitochondrial dysfunction during cardiac injury." (2015). Electronic Theses and Dissertations. Paper 2293.

https://doi.org/10.18297/etd/2293

This Doctoral Dissertation is brought to you for free and open access by ThinkIR: The University of Louisville's Institutional Repository. It has been accepted for inclusion in Electronic Theses and Dissertations by an authorized administrator of ThinkIR: The University of Louisville's Institutional Repository. This title appears here courtesy of the author, who has retained all other copyrights. For more information, please contact thinkir@louisville.edu. 
CO-TREATMENT WITH CONJUGATED LINOLEIC ACID AND NITRITE MODULATES MITOCHONDRIAL RESPIRATION AND ELECTRON TRANSPORT CHAIN ACTIVITY IN VIVO AND ATTENUATES MITOCHONDRIAL DYSFUNCTION DURING CARDIAC INJURY

\author{
By \\ Patrick Van Hoose \\ B.S., Spring Hill College, 2011
A Dissertation
Submitted to the Faculty of the School of Medicine of the University of Louisville In Partial Fulfillment of the Requirements
For the Degree of \\ Doctor of Philosophy in Biochemistry and Molecular Biology \\ Department of Biochemistry and Molecular Genetics \\ University of Louisville \\ Louisville, Kentucky
}

December 2015 


\section{Copyright 2015 by Patrick Van Hoose}

All rights reserved 

CO-TREATMENT WITH CONJUGATED LINOLEIC ACID AND NITRITE MODULATES MITOCHONDRIAL RESPIRATION AND ELECTRON TRANSPORT CHAIN ACTIVITY IN VIVO AND ATTENUATES MITOCHONDRIAL DYSFUNCTION DURING CARDIAC INJURY

\section{By}

Patrick Van Hoose

B.S., Spring Hill College, 2011

A Dissertation Approved on

November 17th, 2015

by the following Dissertation Committee:

Dissertation Director: Dr. Marsha Cole

\begin{tabular}{c}
\hline Dr. Brad Hill \\
\hline Dr. Barbara Clark \\
\hline
\end{tabular}

Dr. Alan Cheng

Dr. Christine Schaner-Tooley 


\section{DEDICATION}

To my parents and family for their love and unwavering support. 


\section{ACKNOWLEDGEMENTS}

I would first like to acknowledge my mentor Dr. Marsha Cole. She has been an outstanding mentor and provided me with excellent training and support, while challenging me to grow as a scientist. Without her none of this work would have been possible and I owe her the sincerest of thanks. I would like to thank my fellow lab mates, Kellianne Piell and Natia Kelm as both contributed greatly to this work, along with Catherine Cobb and Linda Omer for their support throughout. I would also like to thank my committee members: Dr. Brad Hill, Dr.

Barbara Clark, Dr. Alan Cheng and Dr. Christine Schaner-Tooley, for their guidance and insight. Lastly, I acknowledge the Department of Biochemistry and Molecular Genetics for providing a superb environment to train and grow as a graduate student. 


\author{
ABSTRACT \\ CO-TREATMENT WITH CONJUGATED LINOLEIC ACID AND NITRITE \\ MODULATES MITOCHONDRIAL RESPIRATION AND ELECTRON \\ TRANSPORT CHAIN ACTIVITY IN VIVO AND ATTENUATES \\ MITOCHONDRIAL DYSFUNCTION DURING CARDIAC INJURY
}

Patrick Van Hoose

November 17th, 2015

\begin{abstract}
Cardiovascular disease and subsequent complications, such as myocardial infarction (MI), is the leading cause of death within the United States. Clinical intervention to reduce cardiac damage following myocardial ischemia is limited. Polyunsaturated fatty acids have been linked to an overall beneficial effect on cardiovascular health and function. Conjugated linoleic acid (CLA) is an 18:2 unsaturated fatty acid found within the diet in ruminant meat and dairy products. The cis-9 trans-11 isomer of CLA is the most prominent isomer within the diet and CLA has been linked to favorable outcomes in numerous disease states. The mechanism mediating the effects of CLA are still unclear but mitochondria appear to be playing a prominent role. Nitrite $\left(\mathrm{NO}_{2}{ }^{-}\right)$is a dietary source of nitric oxide (NO), as it is found within green leafy vegetables and it is linked to cardioprotection in ischemia reperfusion injury models. Alterations in
\end{abstract}


mitochondrial function and electron transport chain enzymatic activity have been elucidated as the mechanisms mediating nitrite cardioprotection. However, recent clinical trials have reported no positive outcome in patients undergoing $\mathrm{MI}$ who have been supplemented with nitrite. A combination treatment of CLA and nitrite preserves cardiac function following $\mathrm{Ml}$, highlighting that the interaction between dietary constituents may influence cardioprotective effects. In the first step of this study, we examine whether the combination treatment of CLA and nitrite influenced mitochondrial respiration and electron transport chain activity under baseline conditions. In the second step of this study, we examine the impact of co-treatment with CLA and nitrite on mitochondrial function following $\mathrm{Ml}$ and aging. Under baseline conditions, we found that co-administration of CLA and nitrite decreased mitochondrial respiration and complex III activity. Following MI, the co-treatment of CLA and nitrite attenuated Ml-induced increases in mitochondrial respiration, complex III activity, and hydrogen peroxide levels. However, following aging the co-treatment was unable to restore aging induced mitochondrial dysfunction. These results demonstrate that in combination the dietary constituents, cLA and nitrite, are able to influence mitochondrial function under non-injury setting and following MI. Overall, this supports a role of mitochondria in the cardioprotective effects mediated by a combination treatment of CLA and nitrite. 


\section{TABLE OF CONTENTS}

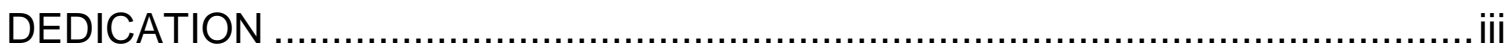

ACKNOWLEDGEMENTS...........................................................................

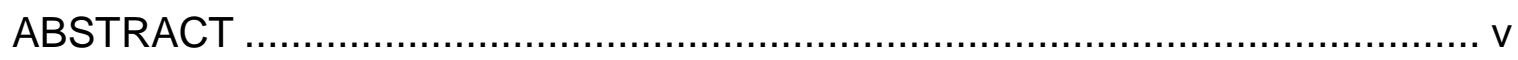

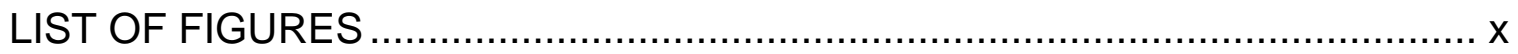

CHAPTER

I. Introduction: Cardiovascular disease, mitochondria and nitrated fatty acids....................................................... 1

Cardiovascular Disease .................................................. 1

Mitochondria ....................................................................... 5

Nitrite ............................................................................ 18

Conjugated Linoleic Acid ............................................. 26

Nitrated Fatty Acids ........................................................ 31

Hypothesis and Research Strategy .................................. 34

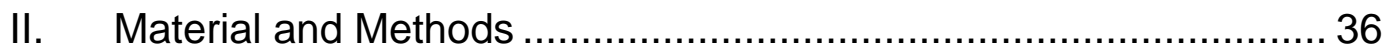

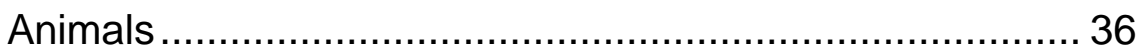

Experimental Design..................................................... 36

Blood Glucose Measurements ....................................... 37

Mouse Model of Myocardial Infarction .............................. 37

Heart Mitochondrial Isolation ........................................... 38

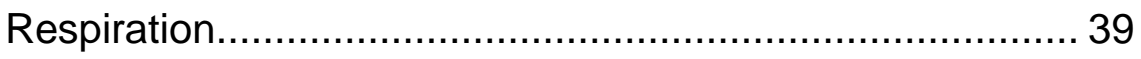


Complex I Activity Assay ................................................... 39

Complex II Activity Assay................................................. 40

Complex III Activity Assay................................................. 40

Complex IV Activity Assay ............................................... 41

Western Blot Analysis ..................................................... 41

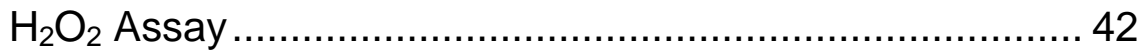

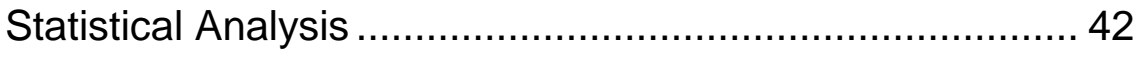

III. Combination treatment with CLA and nitrite in vivo changes mitochondrial function ................................................ 43

Chapter Overview ........................................................ 43

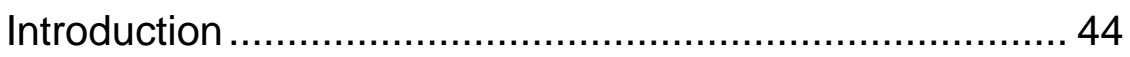

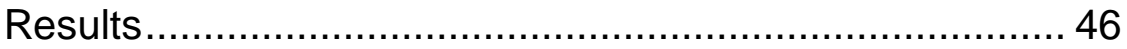

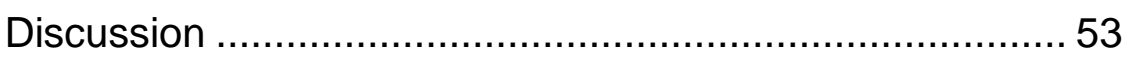

IV. Co-treatment of CLA and nitrite in vivo attenuates cardiac mitochondrial dysfunction following myocardial

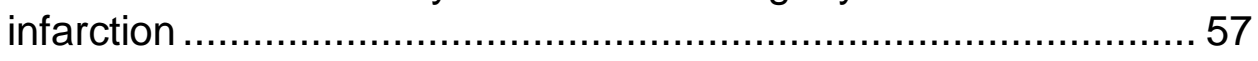

Chapter Overview ..................................................... 57

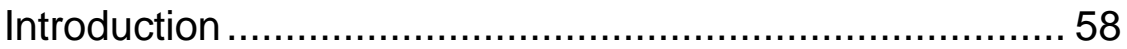

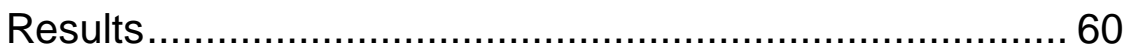

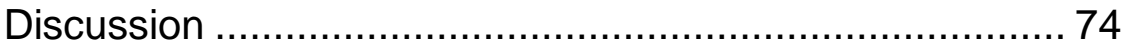

V. Cardiac mitochondrial function and aging ................................. 79

Chapter Overview ….................................................. 79

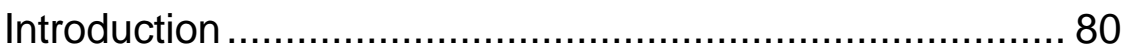

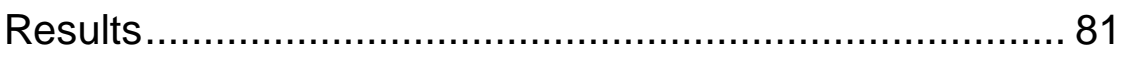

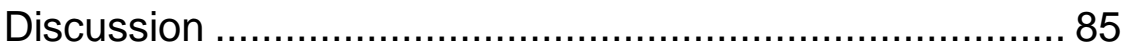




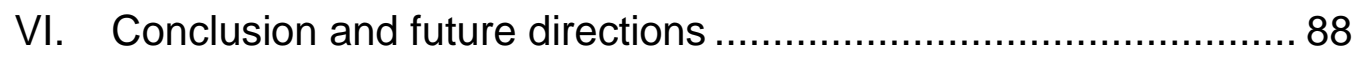

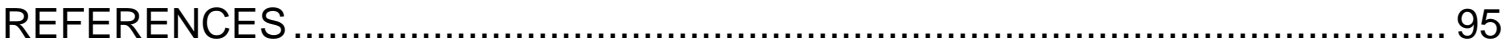

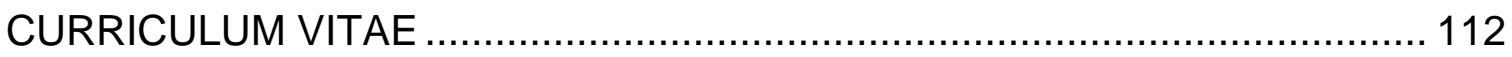




\section{LIST OF FIGURES}

\section{FIGURE}

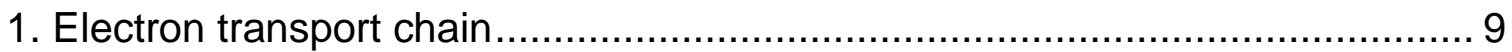

2. Superoxide generated by mitochondria ….............................................. 16

3. Mitochondrial targets of nitric oxide and nitrite............................................. 24

4. Cis-9 trans-11 isomer of conjugated linoleic acid ...................................... 27

5. Mitochondrial respiration protocol .......................................................... 47

6. Treatment with $\mathrm{CLA}$, nitrite or in combination does not alter animal weight and

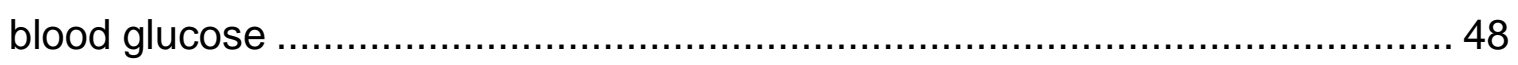

7. State 3 respiration decreases following with CLA treatment ......................... 49

8. Co-treatment with $\mathrm{CLA}$ and nitrite decreases complex II and III activity, while

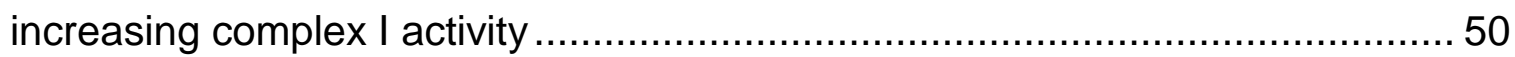

9. Mitochondrial respiratory chain protein subunit levels remain unchanged following treatment with $\mathrm{CLA}$, nitrite or combination treatment with $\mathrm{CLA}$ and nitrite

10. Mitochondrial respiration remains unchanged following co-treatment with $\mathrm{CLA}$ and nitrite

11. Co-treatment with CLA and nitrite increases complex III activity and nitrite

increases $\mathrm{H}_{2} \mathrm{O}_{2}$ levels.

12. Co-treatment with CLA and nitrite decreases NDUFB8 protein expression .. 65

13. Nitrite attenuates increases in State 3 and ETS respiration following MI..... 67

14. Co-treatment with cLA and nitrite attenuates increased complex III 
activity following $\mathrm{MI}$

15. Treatment with $\mathrm{CLA}$, nitrite or combination increases mitochondrial respiratory chain protein expression following $\mathrm{MI}$ 70

16. Treatment with $\mathrm{CLA}+$ nitrite increases MnSOD protein expression following

$\mathrm{MI}$ 72

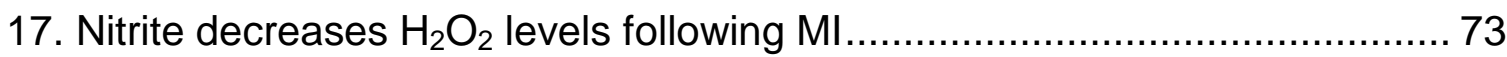

18. Aging induces cardiac mitochondrial dysfunction .................................... 82

19. Treatment with CLA, nitrite or in combination does not attenuate aged related decline in cardiac mitochondrial function 83 


\section{CHAPTER I}

\section{INTRODUCTION: CARDIOVASCULAR DISEASE, MITOCHONDRIA, AND NITRATED FATTY ACIDS}

\section{Cardiovascular Disease Statistics}

Cardiovascular disease (CVD) is the leading cause of death globally, accounting for more than 17.3 million deaths annually and representing an economic cost of $\$ 863$ billion as of 2010 (1). CVD affects 85.6 million Americans and represents approximately $31.3 \%$ of all deaths in the United States (US) (1). The estimated direct and indirect cost of CVD within the US as of 2011 was estimated at $\$ 320.1$ billion and the direct cost of CVD is expected to increase to over $\$ 918$ billion by 2030 (1). Coronary heart disease (CHD) is the most common type of CVD, making up approximately half of all CVD deaths. An estimated 15.5 million US adults have CHD and the morality associated with CHD was 375,295 in the U.S. in 2011(1). A common complication of CHD is myocardial infarction (MI) and the estimated annual incidence of $\mathrm{MI}$ is 525,000 new attacks and 210,000 recurrent attacks within the U.S. (1).

Coronary heart disease is the result of atherosclerosis, a progressive disease stemming from inflammation of intima of the coronary arteries leading to 
gradual thickening of the intima (2). Risk factors for coronary artery disease include a high level of low density lipoproteins (LDL), high blood cholesterol levels, family history, diabetes, obesity, smoking and age. Atherosclerosis can be broken down into several stages: 1) endothelial dysfunction, 2) oxidized LDL and macrophage accumulation, 3) foam cell formation and smooth muscle recruitment, 4) vascularization, 5) necrotic core and 6) plaque rupture. Endothelial dysfunction caused by low wall shear stress is a key initiation event for atherosclerosis (3) leading to decreased nitric oxide (NO) and increased chemokine and pro-inflammatory mediators (4). Increased expression of adhesion molecules (5), the scavenger receptor CD-36 (6), and proteoglycans (7) lead to recruitment of monocytes and oxidized LDL within the intima. Monocytes differentiate into macrophages and ingest oxidized LDL forming foam cells. Foam cells secrete pro-inflammatory cytokines and recruit smooth muscle cells (3) to the intima which also take up lipids creating a lipid rich core within the plaque. Neovascularization of the plaque by leaky vasculature from the adventitial vasa vasorum leads to further monocyte recruitment (3) and increases plaque size (8). Eventually the plaque forms a necrotic core due to macrophage (9), foam cell and smooth muscle cell (3) apoptosis. The loss of smooth muscle cell secretion of collagen, elastin, and proteoglycans leads to plaque cap thinning. Cap thinning can lead to plaque rupture (10), exposing the thromobogenic plaque core to platelets within blood, thereby initiating platelet aggregation, vasoconstriction, and fibrin deposition (8), which leads to occlusion 
of a coronary artery (2). Disruption in blood and oxygen flow to the myocardium results in MI, a major healthcare consequence of coronary heart disease.

$\mathrm{Ml}$ is defined as myocardial cell death due to prolonged ischemia and is the result of an imbalance between oxygen supply and demand (11). Ischemic injury can be divided into three phases: 1) ischemic 2) reperfusion and 3) reperfusion repair (12). The lack of oxygen of during ischemia results in cardiomyocyte cell death via apoptosis and necrosis (13). Apoptosis is a programmed cell death pathway characterized by membrane blebbing, cell shrinkage, nuclear fragmentation and chromatin condensation (14). Apoptosis is activated via two distinct pathways: the extrinsic and intrinsic pathway. The extrinsic pathway is mediated by proinflammatory ligands such as tumor necrosis factor alpha (TNF- $\alpha$ ) and tumor necrosis factor ligand super family member six (15) which activate plasma membrane receptors. The intrinsic pathway is mediated by release of mitochondrial death factors including cytochrome $\mathrm{c}$, high temperature requirement $\mathrm{A} 2(\mathrm{H} \operatorname{tr} \mathrm{A} 2)$ apoptosis inducing factor and second mitochondrial derived activator of caspase (SMAC). The downstream target of both the intrinsic and extrinsic pathways is caspase-3, a cysteine protease, which degrades proteins and activates deoxyribonucleases (DNases) to degrade deoxyribonucleic acid (DNA) (14). Apoptosis is an energy requiring process so cardiomyocytes within the border zone of the infarct undergo apoptosis and cardiomyocytes within the infarct zone undergo necrosis due to depletion of adenosine triphosphate (ATP). 
Necrosis involves whole cell swelling and loss of plasma membrane integrity (14) resulting from decreased ATP levels needed to maintain ionic gradients at the plasma membrane. Upon plasma membrane rupture cellular contents initiate an inflammatory response within the myocardium resulting in migration of macrophages, monocytes, and neutrophils into the infarct zone (16). Macrophages secrete growth factors leading to conversion of fibroblasts to myofibroblasts that secrete collagen to preserve ventricular integrity. The hypoxic environment occurring during $\mathrm{Ml}$ sets the stage for cell death and reperfusion of ischemic cardiac tissue is necessary to salvage the myocardium; however, reperfusion also contributes to injury (17). The reoxygenation of myocardium results in significant generation of reactive oxygen species (ROS) which can trigger inflammation and damage proteins, lipids, and DNA, ultimately leading to cellular dysfunction and death. ROS generation within the myocardium also plays a protective role by generating growth factors that promote angiogenesis, promoting activation of vascular smooth muscle cells and matrix metalloproteinases (MMPs) that are necessary for the reparative phase (12). MMPs degrade intermyocyte collagen thereby expanding the infarct resulting in ventricular dilation leading to ventricular dysfunction (18). Overall, MI leads to a complex adaptive response, resulting in an attempt to salvage myocardium and preserve heart function. However, the end result of $\mathrm{Ml}$ is cardiac dysfunction. Two prevailing details of $\mathrm{Ml}$ induced cardiac dysfunction, cardiomyocyte cell death and ROS generation, share a common link: mitochondria. 


\section{Mitochondria}

\section{History}

Mitochondria arose from bacteria and developed a symbiotic relationship within cells (19). Mitochondria are membrane organelles contained within cells that carry out fundamental metabolism reactions necessary for cell survival. Mitochondria can also initiate apoptosis placing mitochondria at the interface between cell survival and cell death. The citric acid cycle (TCA cycle), betaoxidation of fatty acids and oxidative phosphorylation via the electron transport chain occur within mitochondria and these are essential metabolic pathways providing energy for the cell.

\section{Function}

\section{Citric acid cycle}

The TCA cycle was proposed by Hans Adolf Krebs in 1937 and is an oxidative pathway for the metabolism of carbohydrates, fats and amino acids. Eight enzymes comprise the TCA cycle that results in the oxidation of acetylCoenzyme A (acetyl-CoA) resulting in production of three nicotinamide adenine dinucleotide reduced form (NADH), one flavin adenine nucleotide reduced form $\left(\mathrm{FADH} \mathrm{H}_{2}\right)$, and one guanosine triphosphate (GTP) (20). The first reaction of the TCA cycle is the condensation of acetyl-CoA with oxaloacetate catalyzed by citrate synthase producing citrate. Citrate is then isomerized to isocitrate by aconitase and isocitrate is converted to alpha ketogluturate by isocitrate 
dehydrogenase generating one $\mathrm{NADH}$. Alpha ketoglutarate dehydrogenase then performs an oxidative decarboxylation reaction converting alpha ketoglutarate to succinyl-CoA, producing one NADH. Succinyl-CoA then undergoes a reaction with succinic thiokinase producing one GTP. Succinate dehydrogenase then converts succinate to fumarate producing $\mathrm{FADH}_{2}$ and this is followed by hydration of fumarate to malate by fumarase. The last reaction of the TCA cycle is the dehydrogenation of malate by malate dehydrogenase producing oxaloacetate, which can undergo condensation with another acetyl-CoA thereby starting another round of the TCA cycle. The NADH and $\mathrm{FADH}_{2}$ produced during the TCA cycle can donate electrons to the electron transport chain contributing to the production of adenosine triphosphate (ATP) via oxidative phosphorylation. An important source of acetyl-CoA for the TCA cycle is the beta oxidation of fatty acids.

\section{Beta Oxidation of Fatty Acids}

Fatty acids enter into mitochondria via the carnitine palmitoyl carrier protein in which carnitine palmitoyl transferase I removes the CoA group on the fatty acid and attaches a carnitine moiety to a free fatty acid in the cytosol, which allows the fatty acid to pass through the carnitine carrier protein. Once inside the mitochondrial matrix, the carnitine moiety is removed by carnitine palmitoyl

transferase II, and the CoA is reattached to the fatty acid. The beta oxidation of fatty acids occurs via four reactions catalyzed by acyl-CoA dehydrogenase, 
enoyl-CoA hydratase, 3-L-hydroxyl-CoA dehydrogenase, and B-ketoacyl-CoA thiolase. Acyl-CoA dehydrogenase catalyzes dehydrogenation of the fatty acylCoA creating a trans-alpha-beta double bond with the subsequent generation of $\mathrm{FADH}_{2}$, which enters the ETC via the electron-transfer flavoprotein (ETF) and the ETF:ubiquinone oxidoreductase. Enoyl-CoA hydratase then catalyzes the hydration of the trans-alpha-beta bond followed by NAD+ dependent dehydrogenation by 3-L-hydroxyl-CoA dehydrogenase generating NADH, which can enter into the ETC. B-ketoacyl-CoA thiolase cleaves the alpha and beta carbon to form acetyl-CoA, which can enter the TCA cycle, and a new acyl-fatty acid with two fewer carbon atoms, that can undergo another round of beta oxidation.

Mitochondria also contain additional enzymes for the oxidation of unsaturated fatty acids including: 1.enoyl-CoA isomerase, which re-positions a beta-gamma double bond into an alpha-beta position, 2. 2,4-dienoyl-CoA reductase and 3,2-enoyl-CoA isomerase, which catalyze the removal of a double bond at an even numbered carbon and 3. 3,5-2,4-dienoyl-CoA isomerase, which catalyzes a reversible reaction that interconverts trans- 2 and trans- 3 double bonds. Overall, one round of beta oxidation of fatty acids results in the production of acetyl-CoA, a substrate for the TCA cycle and one each of $\mathrm{FADH}_{2}$ and $\mathrm{NADH}$, which can enter the ETC leading to the production of ATP.

\section{Electron Transport Chain}


The electron transport chain (ETC) is embedded in the inner mitochondrial membrane and is responsible for the majority of ATP production within the cell. The ETC is composed of 5 different protein complexes: NADH-coenzyme Q oxidoreductase (complex I), succinate-coenzyme Q oxidoreductase (complex II), coenzyme Q-cytochrome c oxidoreductase (complex III), cytochrome c oxidase (complex IV) and ATP synthase (complex V). Together complex I, II, III and IV transfer electrons to oxygen $\left(\mathrm{O}_{2}\right)$, the final electron acceptor while also pumping hydrogen ions into the mitochondrial inner membrane space creating an electrochemical gradient. ATP synthase uses this electrochemical gradient for the phosphorylation of adenosine diphosphate (ADP) to ATP, thus the ETC couples electron transport to oxidative-phosphorylation for the production of ATP (Fig. 1).

\section{Complex I}

Complex I, comprised of 46 subunits, is an L-shaped protein with one arm embedded in the inner mitochondrial membrane and the other arm extended into the mitochondrial matrix. Complex I transfers electrons from NADH to coenzyme ubiquinone (CoQ), a hydrophilic electron carrier within the mitochondrial inner membrane. Complex I contains a flavin mononucleotide (FMN) and nine iron sulfur clusters that participate in the transfer of electrons from NADH to CoQ via a mechanism according to the reduction potential of the various redox centers within complex I (21). FMN and CoQ can take up two oxidation states as both 


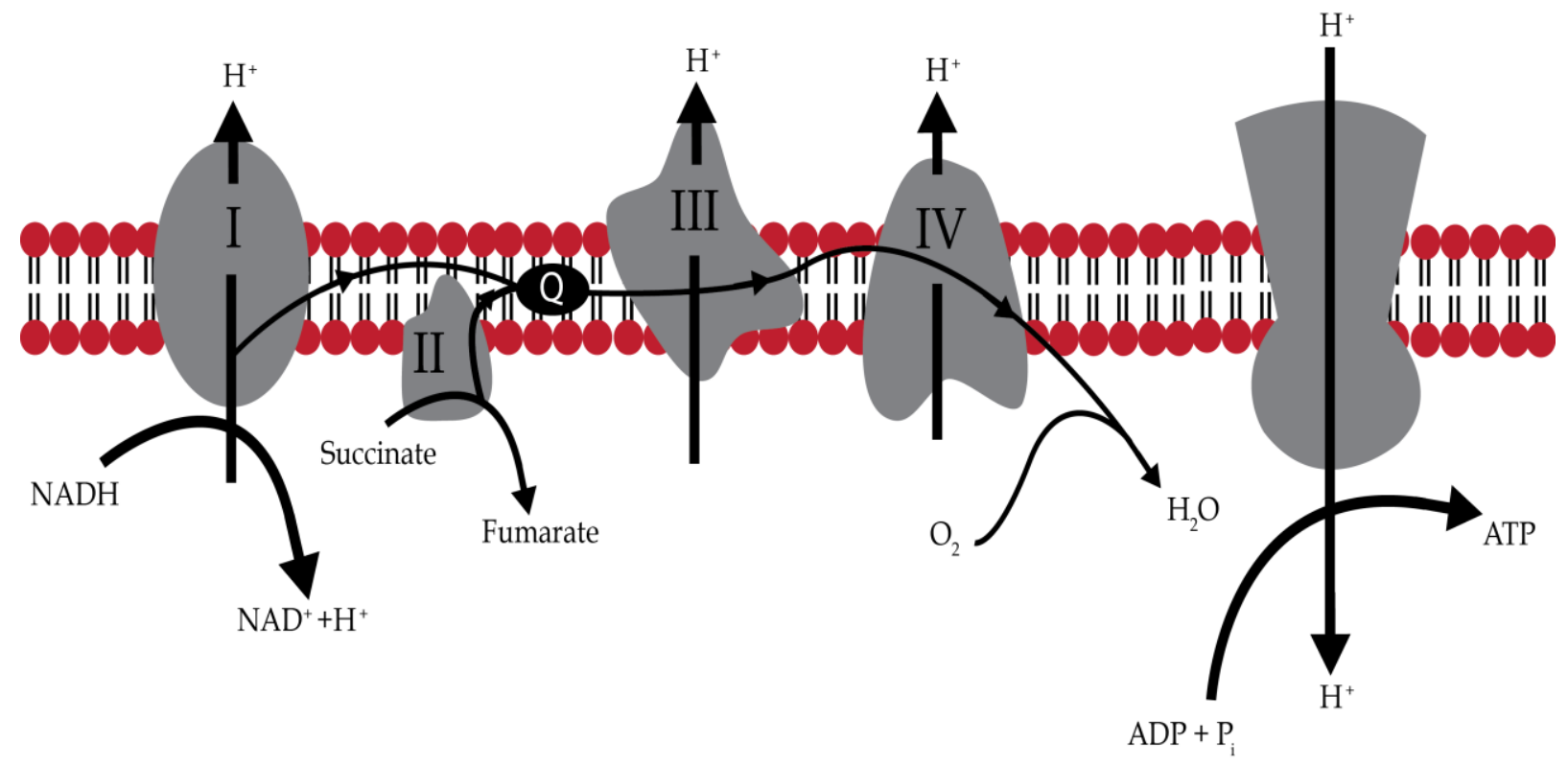

Figure 1. Electron transport chain. The mitochondrial electron transport chain is composed of complex I, II, III, IV and ATP synthase. Electrons are carried down the chain to the final electron acceptor oxygen and protons are pumped by complex I, III and IV. ATP synthase uses the proton gradient to convert ADP to ATP. 
form stable semiquinone radicals allowing both to donate either one or two electrons thus allowing NADH to transfer two electrons. In addition, to the transfer of electrons complex I also pumps 4 hydrogen ions into the inner membrane space contributing to the electrochemical gradient.

\section{Complex II}

Complex II contains the TCA cycle enzyme succinate dehydrogenase and transfers electrons from succinate to CoQ. Complex II transfers electron from succinate to FAD initially followed by passage through three iron sulfur clusters, one cytochrome b560 and ultimately to CoQ. Complex II does not transverse the inner mitochondrial membrane or translocate hydrogen ions (22).

\section{Complex III}

Complex III transfers electrons from reduced $\mathrm{CoQ}$ to cytochrome c while pumping 4 hydrogen ions into the inner membrane space, via the $Q$ cycle (23). In the first step of the $Q$ cycle, reduced CoQ binds to the $Q_{o}$ site where it transfers one electron to the iron-sulfur protein (ISP), containing the Rieske center, while releasing two protons into the inner membrane space. The ISP reduces cytochrome $c_{1}$, while $\mathrm{CoQ}$ semiquinone transfers its remaining electron to

cytochrome $b_{L}$, a b-type cytochrome heme with low redox potential, yielding a fully oxidized $\operatorname{CoQ}(23)$. Cytochrome $b_{\llcorner}$then reduces cytochrome $b_{H}$, a b-type 
cytochrome heme with high redox potential. The oxidized CoQ then is released from the $Q_{0}$ site and binds to the $Q_{i}$ site and receives an electron from cytochrome $b_{H}$ yielding $C o Q$ semiquinone (24). This is the first cycle of the $Q$ cycle, resulting in two hydrogen ions pumped into the inner membrane space, a reduced cytochrome $c_{1}$ and a CoQ semiquinone. The second cycle of the $Q$ cycle, is identical to the first cycle, however the CoQ semiquinone produced in the first cycle accepts an electron from cytochrome $b_{H}$, along with two hydrogen ions from the mitochondrial matrix to produce a reduced CoQ (24). The electrons passed to cytochrome $c_{1}$ are transferred to cytochrome $c$, a peripheral membrane protein that shuttles electrons from complex III to complex IV (24).

\section{Complex IV}

Complex IV catalyzes one-electron oxidations of four cytochrome c molecules to reduce one oxygen molecule to two water $\left(\mathrm{H}_{2} \mathrm{O}\right)$ molecules. Four redox centers, cytochrome a, cytochrome $\mathrm{a}_{3}$, a copper atom known as $\mathrm{Cu}_{\mathrm{B}}$ and a pair of copper atoms known as the $\mathrm{Cu}_{\mathrm{A}}$ center participate in electron transfer of Complex IV (25). The transfer of electrons that occurs is linear from cytochrome c to $\mathrm{Cu}_{A}$, to cytochrome a, then finally to cytochrome $\mathrm{a}_{3}$ and $\mathrm{Cu}_{B}$. The cytochrome $\mathrm{a}_{3}$ and $\mathrm{Cu}_{\mathrm{B}}$ are in close proximity to each other, almost forming a single binuclear complex, where the reduction of $\mathrm{O}_{2}$ to $\mathrm{H}_{2} \mathrm{O}$ occurs. The cytochrome $\mathrm{a}_{3}$ and $\mathrm{Cu}_{\mathrm{B}}$ binuclear complex is reduced by two consecutive electron transfers from cytochrome $\mathrm{c}$ via cytochrome a and $\mathrm{Cu}_{\mathrm{A}}$, with the release 
of $\mathrm{H}_{2} \mathrm{O}$ (25). Molecular oxygen binds to the heme of cytochrome $\mathrm{a}_{3}$ followed by internal electron redistribution in which tyrosine 244 of $\mathrm{Cu}_{\mathrm{B}}$ has donated an electron and proton to the heme cytochrome $a_{3}$, thereby forming a tyrosine radical, and this is known as the $\mathrm{P}$ compound (26). Electron transfer from cytochrome $\mathrm{c}$ along with two protons from the mitochondrial matrix allows tyrosine 244 to revert back to its phenolic state with the release of $\mathrm{H}_{2} \mathrm{O}$. A final electron transfer from cytochrome $\mathrm{c}$ and acquisition of another proton yields an oxidized cytochrome $\mathrm{a}_{3}-\mathrm{Cu}_{\mathrm{B}}$ complex completing the electron transfer cycle (26). Complex IV contributes to the electrochemical proton gradient by consuming four mitochondrial matrix hydrogen ions during the reduction of $\mathrm{O}_{2}$ to $\mathrm{H}_{2} \mathrm{O}$ thereby depleting matrix hydrogen ions and by translocating 4 hydrogen ions into the inner membrane space (26). The $D$ channel extends from the mitochondrial matrix to the cytochrome $\mathrm{a}_{3}-\mathrm{Cu}_{\mathrm{B}}$ center and connects with the exit channel. Together the D-channel and exit channel function to pump hydrogen ions from the matrix into the inner membrane space (25).

\section{ATP synthase}

ATP synthase uses the electrochemical gradient created by complex I, III and IV to synthesize ATP from ADP and phosphate $\left(P_{i}\right)$. ATP synthase is composed of two functional subunits, $F_{0}$ and $F_{1}$, where $F_{0}$ is a transmembrane protein and $F_{1}$ is a peripheral membrane protein (27). The $F_{1}$ subunit is responsible for binding ADP, $\mathrm{P}_{\mathrm{i}}$ and the formation of ATP and is composed of 
three alpha and beta subunits along with a gamma, delta and epsilon subunit (27). The $F_{0}$ subunit binds hydrogen ions and is composed of three transmembrane subunits, a, b and c (27). Hydrogen ions enter a hydrophilic channel between the a subunit and the c-ring where they bind to the c-subunit of the $F_{1}$ subunit causing the $c$-subunit to rotate until it reaches another hydrophilic channel where it releases the hydrogen ion (28). The free energy released by hydrogen ion translocation drives the interconversion of the beta subunit of $F_{0}$ from a $L$ (loose) state to a $T$ (tight) state and an $\mathrm{O}$ (open) state (28). ADP and $\mathrm{P}_{\mathrm{i}}$ bind to the beta subunit of $F_{0}$ during the $L$ state and the free energy provided by hydrogen ion transport causes conformational changes that covert the $L$ state to a T state that catalyzes the formation of ATP (28). In addition, the other beta subunits are converted from a $\mathrm{T}$ state to an $\mathrm{O}$ state and from an $\mathrm{O}$ state to a $\mathrm{L}$ state, allowing ATP to dissociate from the O state (27).

Overall, the ETC of mitochondria provides energy to the cell in the form of ATP by coupling electron transport to oxidative phosphorylation by creating a proton gradient. Mitochondria are essential for cell survival by providing ATP. However, they also play a role in cell death via apoptosis.

\section{Mitochondrial mediated apoptosis}

Intrinsic apoptosis is mediated by mitochondria following an apoptotic stimuli such as DNA damage or endoplasmic reticulum (ER) stress, which activate $B$ cell lymphoma-2 (BCL-2) homology $3(\mathrm{BH} 3)$-only proteins, such as 
BCL-2-associated X protein (BAX) (29) and BCL-2 antagonist or killer (BAK) (30). Upon activation BAX and BAK undergo oligomerization and target to mitochondria, where they induce mitochondrial outer membrane permeabilization (MOMP) (31). MOMP leads to the release of mitochondrial proteins such as cytochrome c, HtrA2 apoptosis inducing factor and SMAC (30). Cytochrome c binds to apoptotic-activating factor 1 (APAF1) leading to formation of the apoptosome, which recruits and activates caspase 9. Caspase 9 cleaves and activates caspase 3 and 7 leading to apoptosis. SMAC and HtrA2 serve to counteract the caspase inhibitory functions of X-linked inhibitor of apoptosis protein (XIAP) allowing for downstream activation of caspases and initiation of apoptosis (31). Regulation of mitochondrial mediated apoptosis during MI is essential in salvaging viable myocardium, thereby limiting cardiac injury (14).

\section{Role of Mitochondria in Myocardial Infarction}

The activation of apoptosis by mitochondria during $\mathrm{Ml}$ results in cardiomyocyte cell death and contributes to cardiac injury (14). Mitochondria also produce ROS during $\mathrm{MI}$, damaging protein, lipids, and DNA leading to cardiac injury. Numerous studies have highlighted a role for mitochondrial dysfunction in CVD including increased mitochondrial DNA damage (32) and decreased mitochondrial respiration (33). Additionally, $\mathrm{MI}$ is associated with mitochondrial dysfunction including increased mitochondrial DNA damage (34), decreased mitochondrial encoded gene transcripts and decreased complex I, III, and IV 
activity (34). Mitochondrial induced ROS generation is a hallmark of ischemic cardiac injury and believed to be the major contributor of cardiac injury. The electron transfer reactions within the ETC can result in the direct transfer of electrons to molecular $\mathrm{O}_{2}$ producing superoxide $\left(\mathrm{O}_{2}{ }^{--}\right)$(Fig. 2) that is dismutated to hydrogen peroxide $\left(\mathrm{H}_{2} \mathrm{O}_{2}\right)$ by mitochondrial superoxide dismutase (MnSOD). Mitochondria therefore produce a basal amount of ROS during respiration. However, during MI there is an increase in ROS generation (18). The lack of oxygen during ischemic injury results in back up of electron flow through the ETC and reoxygenation following reperfusion results in a burst of ROS. Mitochondria contain several enzymes that can produce ROS, including 2-oxoglutarate dehydrogenase $(35,36)$ and pyruvate dehydrogenase (PDH) (37). However, complex I, II and III contribute the majority of ROS (38). Studies limiting ROS production by mitochondria during ischemic injury either by overexpression of antioxidants such as SOD (39) or direct modulation of complex I activity (40), provide cardiac protection highlighting the importance of regulating ROS production during ischemic injury. While mitochondria contribute to cardiac injury during $\mathrm{MI}$ via apoptosis and ROS generation; mitochondria can also provide protection against $\mathrm{Ml}(17)$.

Ischemic preconditioning (IPC) was first demonstrated by Murry et al. in 1986 in which short periods of ischemia could provide protection against a subsequent prolonged ischemic insult (41) and mitochondria play a prominent role in IPC. During IPC mitochondria can produce ROS that serve to activate protective signaling pathways such as nuclear factor erythroid 2-related factor 2 
Figure 2. Superoxide generated by mitochondria.

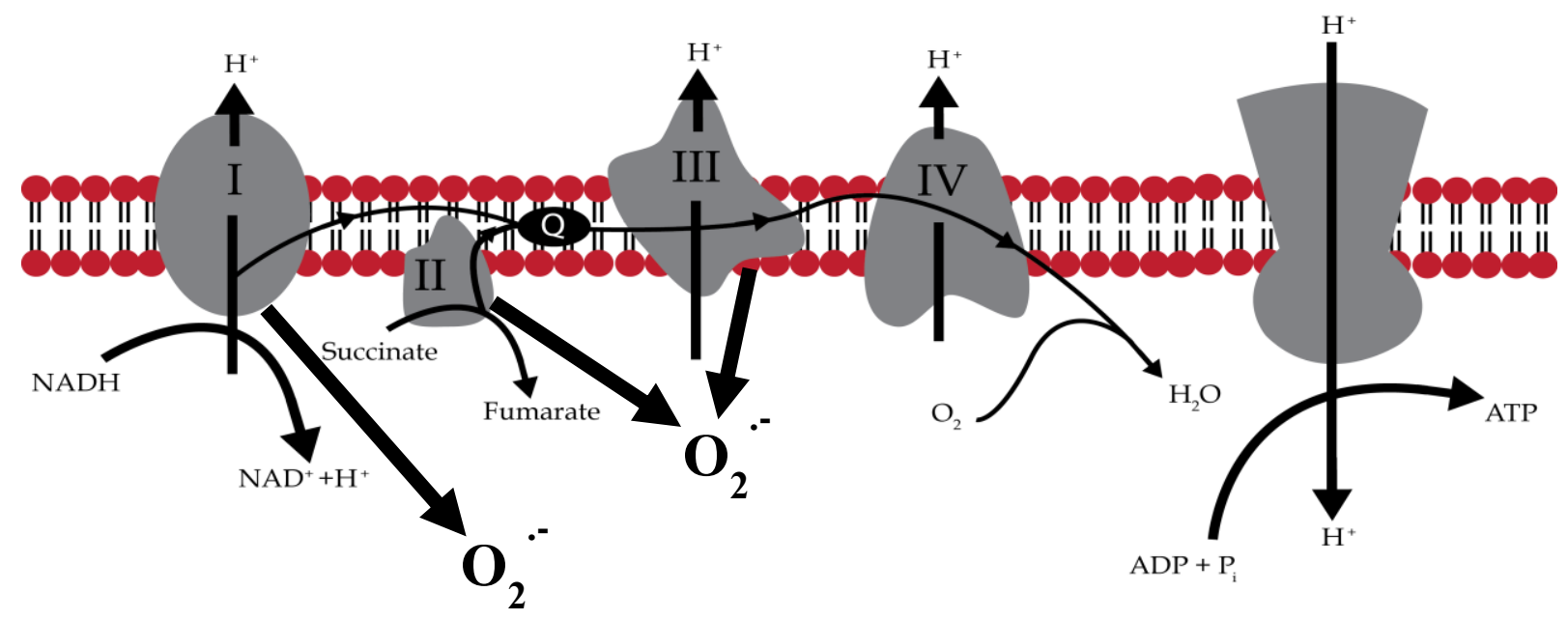

Figure 2. Superoxide generated by mitochondria. Complex I, II, and III generate superoxide $\left(\mathrm{O}_{2}\right)$ during respiration due to electron slippage. During ischemia reperfusion conditions the rate of superoxide generated by complex I, II and III is increased. 
(Nrf2), hypoxia inducible factor 1 alpha (HIF-1a) (42), nuclear factor-kappa B (NFкB) (42), and adenine monophosphate kinase (AMPK) (43) that serve to protect myocardium against ischemic injury, highlighting a protective cell signaling role for mitochondrial ROS. Opening of the mitochondrial potassium-ATP (mitoK $\mathrm{ATP}_{\text {) }}$ channel and mitochondrial permeability transition pore (MPT) during IPC is also associated with cardioprotective effects (43). Alterations in mitochondrial respiration and ATP synthesis via mitochondrial uncoupling $(44,45)$, posttranslational modifications on mitochondrial proteins (complex I and adenine nucleotide translocase-1 (ANT-1)) $(40,46)$, and changes in mitochondrial membrane potential (47) are also associated with IPC cardiac protection. Direct activation of these mitochondrial protective signaling pathways has also been shown to be cardioprotective against ischemic injury showing that mitochondria are critical mediators of cardioprotective signaling $(42,48)$.

\section{Role of Mitochondria in Aging}

Aging is associated with a change in redox status of mitochondria. This change is related to an increase in ROS generation, where the antioxidant capacity within mitochondria becomes less efficient (42). Mitochondrial ROS generation is associated with mitochondrial DNA damage, mitochondrial protein damage and mitochondrial dysfunction in aging. Mitochondrial damage and dysfunction can trigger apoptosis leading to increased cell death during aging. Age is a significant risk factor for $\mathrm{Ml}$ and is also associated with cardiac 
dysfunction. ROS induced mitochondrial damage is seen both in aging and ischemic injury suggesting a similar mechanism of mitochondria induced cardiac dysfunction (42). Importantly, the mitochondrial protective signaling pathways against $\mathrm{Ml}$ also serve to protect against aging induced mitochondrial dysfunction such as MPT opening, AMPK and HIF-1 alpha (42).

\section{$\underline{\text { Nitrite }}$}

\section{History and Sources}

The anion nitrite $\left(\mathrm{NO}_{2}{ }^{-}\right)$was considered an inert end product of $\mathrm{NO}$ oxidation and was used as an indirect measurement of nitric oxide synthase (NOS) activity. However, numerous studies have demonstrated that nitrite can serve as a signaling molecule and an endogenous reservoir of NO (49). The two main sources for $\mathrm{NO}_{2}^{-}$are the oxidation of $\mathrm{NO}$ and the reduction of dietary sources of nitrate $\left(\mathrm{NO}_{3}{ }^{-}\right)$, found in green leafy vegetables and meat, via commensal bacteria in the mouth. Initial data suggested the reduction of nitrite to $\mathrm{NO}$ under conditions of low $\mathrm{pH}$ and hypoxia was responsible for the resulting biological signaling actions (50). However, more recent data demonstrates that nitrite can mediate effects independent of its conversion to $\mathrm{NO}(40,51)$.

\section{Nitric Oxide}

Early studies of nitrite focused on its bioactivation to NO, as NO signaling has been shown necessary for normal cardiovascular health (52). Nitric oxide 
was first discovered in the late $18^{\text {th }}$ century and for 200 years was widely considered to be a toxic gas. In the 1980s, it was discovered that NO was generated in mammals by nitric oxide synthases (53). There are three forms of nitric oxide synthases (NOSs): the endothelial nitric oxide synthase (eNOS), the neuronal nitric oxide synthase (nNOS) and inducible nitric oxide synthase (iNOS). NOSs are composed of homodimers (53). Each dimer contains a reductase and oxygenase domain that catalyzes a five electron transfer reaction and results in the conversion of L-arginine and molecular oxygen to L-citrulline with the concomitant production of NO (53). eNOS is found within the vascular endothelium, and the production of NO from eNOS is important for cardiovascular health, as NO maintains vascular tone and endothelial integrity (54).

NO can mediate biological signaling through three different pathways due to its free radical nature and high reactivity with transition metals and other free radicals. Direct interaction with ferrous heme proteins, direct diffusion-limited reactions with radicals, lipid peroxides and thiol radicals to form S-nitrosothiols and oxidative reactions with oxygen or ROS; represent the three distinct pathways in which NO can exert biological effects (55). Nitric oxide reactions occur with heme proteins and other radicals at low concentrations (picomolar to nanomolar) of NO due to the high affinity of NO for these compounds, while higher concentrations of $\mathrm{NO}$ and ROS are needed to form reactive nitrogen species such as nitrosonium $\left(\mathrm{NO}^{+}\right)$, nitrogen dioxide $\left(\mathrm{NO}_{2}\right)$ or dinitrogen trioxide $\left(\mathrm{N}_{2} \mathrm{O}_{3}\right)$. 
Nitric oxide's affinity for ferrous heme molecules leads to formation of an iron-nitrosyl complex and such binding activates soluble guanylate cyclase (sGC) (56). SGC activation leads to generation of the second messenger cyclic guanosine 3'-5'-monophosphate (cGMP), which activates protein kinase G (PKG) leading to relaxation of vascular smooth muscle and vasodilation within blood vessels (54). The NO-sGC-cGMP signaling cascade is the classical pathway by which NO facilitates regulation of vasomotor tone within the cardiovascular system (56). However, NO can also mediate a wide variety of effects including angiogenesis (56), mitochondrial biogenesis (56) and modulation of mitochondrial function (57). Conditions of increased oxidative stress, such as inflammation, $\mathrm{Ml}$, and aging, can impact $\mathrm{NO}$ signaling by decreasing NO availability and desensitizing sGC to $\mathrm{NO}(42)$. Increased $\mathrm{O}_{2}-$ production during oxidative stress can react rapidly with $\mathrm{NO}$ to form peroxynitrite (ONOO-) thereby reducing NO concentrations (55).

Oxidative stress can also oxidize eNOS via glutathionylation (58) and reduce tetrahydrobiopterin $(\mathrm{BH} 4)(58)$, a redox sensitive co-factor for eNOS. The loss of $\mathrm{BH} 4$ leads to uncoupling of eNOS, allowing electrons from NADPH to be passed to oxygen resulting in superoxide formation instead of NO (58). The ferrous heme of $S G C$ can be oxidized to ferric heme resulting in $S G C$ that is resistant to NO, thereby decreasing NO signaling (58). Loss of nitric oxide signaling and subsequent endothelial dysfunction is a hallmark of aging (42) and loss of nitric oxide in animals models of MI via NOS inhibition (52) or NOS genetic knockout (52) results in worsening cardiac injury. These studies highlight 
a protective role of $\mathrm{NO}$ in aging and $\mathrm{Ml}$ and the protection afforded by $\mathrm{NO}$ appears to be in part mediated by mitochondria.

Activation of the NO-sGC-cGMP pathway leads to opening of the mitoK $\mathrm{K}_{\text {ATP }}$ channel leading to reduced calcium overload in mitochondria (59). Inhibition of MPT opening, thereby decreasing cytochrome c release and apoptosis (60) has also been attributed to nitric oxide. NO has also been shown to inhibit mitochondrial complex I (51), thereby decreasing ROS production and allowing diffusion of oxygen to ischemic tissue providing protection against ischemic injury. The hypoxic and acidic conditions associated with ischemic injury renders NOSs uncoupled leading to increased ROS and diminished NO production thereby limiting the therapeutic potential of NO against MI (52). However, nitrite represents an endogenous storage pool of NO (49).

\section{Nitrite Reduction}

Nitrite can be reduced to nitric oxide under conditions of low $\mathrm{pH}$, as was first noted by Furchgott (50) as acidified sodium nitrite solutions were able to mediate transient relaxation of rabbit aorta very similar to NO gas. Nitrite can be converted to NO in the acidic conditions such as the gastric lumen via nonenzymatic reaction. Nitrite is protonated $\left(\mathrm{HNO}_{2}\right)$ and decomposes to $\mathrm{N}_{2} \mathrm{O}_{3}$, giving rise to $\mathrm{NO}$ and $\mathrm{NO}_{2}$ (61). Nitrite can also be reduced to $\mathrm{NO}$ under hypoxic conditions by deoxygenated hemoglobin, where nitrite reacts with the ferrous hemoglobin and a proton to produce NO and methemoglobin (62). This reduction is highly dependent on low oxygen and low pH conditions, which arise during MI. 
Nitrite reduction to NO can be catalyzed by several enzymes including: myoglobin (63), neuroglobin (64), cytochrome c (65), complex IV (66), eNOS (67) and xanthine oxidoreductase (68). While the exact mechanisms of nitrite reduction for all these enzymes is unknown, the prevailing conditions of low $\mathrm{pH}$ and hypoxia increase the reaction rate of nitrite reduction. The reduction of nitrite to NO, allows nitrite to mediate a variety of effects including: NO-sGC-cGMP signaling (69), metabolic signaling (70), vasodilation (62) and most importantly protection against ischemia reperfusion injury $(40,71-73)$.

\section{Nitrite and Myocardial Infarction}

Nitrite was first shown to be cardioprotective in a Langendorff model of Ml by decreasing infarct size (74). Abolishing NO signaling negates the protective effect of nitrite suggesting that reduction of nitrite to NO is required for cardioprotection as deletion of myoglobin, a nitrite reductase, in mice resulted in blunting of nitrite cardioprotection (75). This reinforces that nitrite reduction to NO is necessary for nitrite-mediated cardioprotection. Nitrite has also been shown to be protective in other models of ischemic injury including liver (76), brain (77), and hindlimb ischemia (78) with all of these studies indicating the reliance on nitrite reduction to NO. Nitrite also appears to be involved in the protection conferred by IPC as blood and tissue nitrite concentrations increase following IPC (79). Together these studies demonstrate that nitrite given during the acute phase of ischemia mediates protection against ischemic injury with mitochondria appearing to mediate this protection. 


\section{Nitrite and Mitochondria}

Mitochondria contain several components that can serve to reduce nitrite to NO including complex III (80), complex IV (66), and cytochrome c (65). Nitrite can also serve to modulate mitochondrial function either directly via posttranslational modifications on complex I (40) and complex IV (81) or indirectly via mitochondrial biogenesis (82) and mitochondrial fusion (83, 84). Complex I induced ROS generation during the reperfusion phase of IR injury is a critical component of IR tissue injury and inhibition of complex I (85) has shown to be protective. Complex I activity has been shown to decrease with nitrite treatment during ischemia (73) and this decrease in complex I activity resulted in suppressed mitochondrial respiration ultimately leading to protection against IR injury (73) (Fig. 3).

A recent study by Chouchani et al. followed up on this nitrite-mediated

decrease in complex I, and demonstrated that S-nitrosylation on cysteine 39 of the ND3 subunit was responsible for the decreased complex I activity (40). During ischemia when levels of $\mathrm{NADH}$ and oxygen are low, complex I activity is decreased and the ND3 subunit undergoes a conformational change exposing cysteine 39 (40). Nitrite and an exogenous NO donor given prior to ischemia are able to S-nitrosylate cysteine 39 and this modification decreases complex I activity, thereby attenuating ROS generated by complex I during reperfusion (40). Importantly, the S-nitrosylation on cysteine 39 is a transient, reversible modification allowing for complex I enzymatic activity to return following 


\section{Figure 3. Mitochondrial targets of nitric oxide and nitrite.}

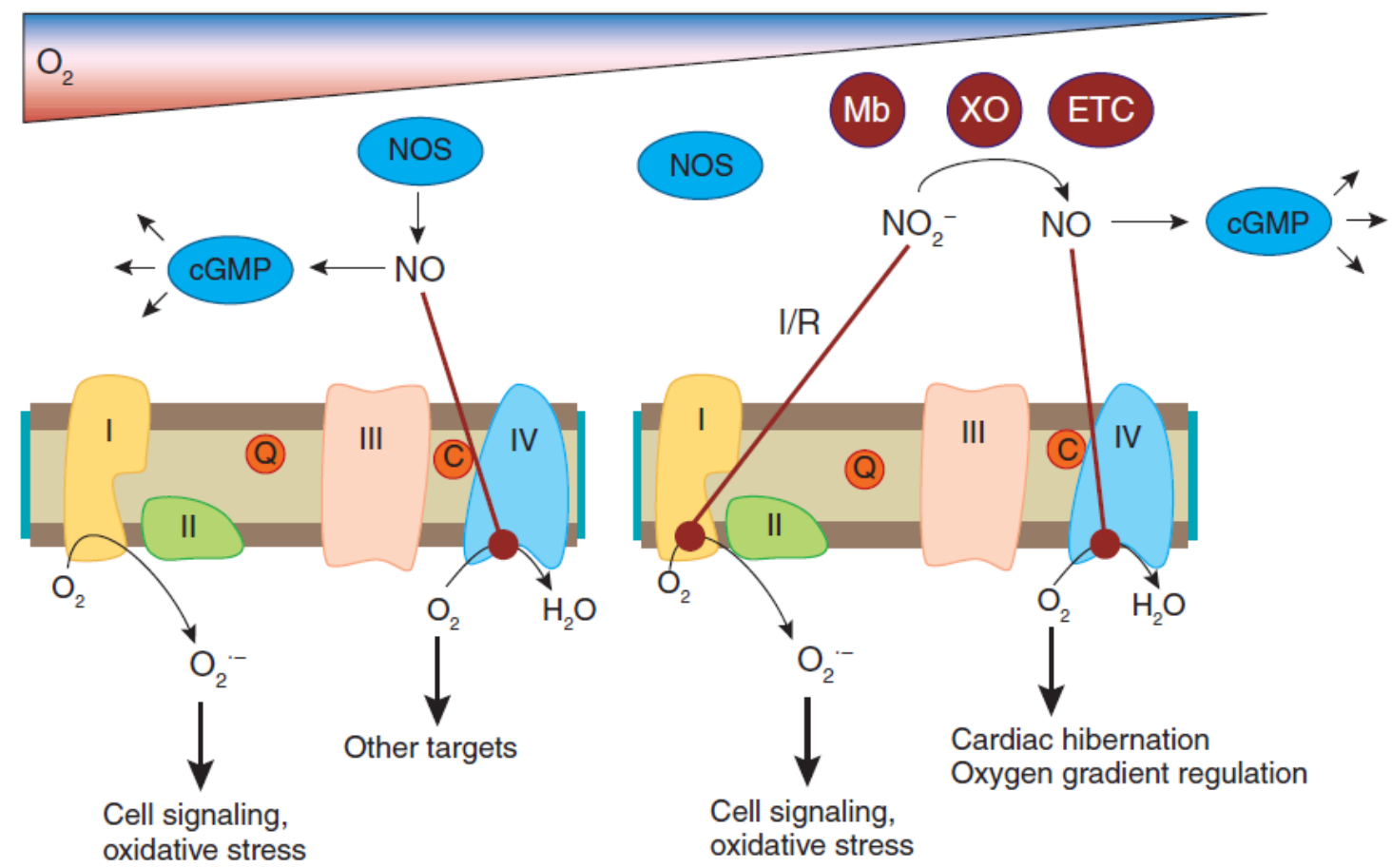

Reprinted by permission from Macmillan Publishers Ltd: [Nature Chemical Biology] (Lundberg, J. O., M. T. Gladwin, A. Ahluwalia, N. Benjamin, N. S. Bryan, A. Butler, P. Cabrales, A. Fago, M. Feelisch, P. C. Ford, B. A. Freeman, M.

Frenneaux, J. Friedman, M. Kelm, C. G. Kevil, D. B. Kim-Shapiro, A. V. Kozlov, J. R. Lancaster, Jr., D. J. Lefer, K. McColl, K. McCurry, R. P. Patel, J. Petersson, T. Rassaf, V. P. Reutov, G. B. Richter-Addo, A. Schechter, S. Shiva, K. Tsuchiya, E. E. van Faassen, A. J. Webb, B. S. Zuckerbraun, J. L. Zweier, and E. Weitzberg. 2009. Nitrate and nitrite in biology, nutrition and therapeutics. Nature Chemical Biology 5: 865-869.) Copyright (2009)

Reproduction Lic\# 3759071042116

Figure. 3. Mitochondrial targets of nitric oxide and nitrite. During normoxic conditions nitric oxide (NO) mediates cyclic GMP (cGMP) signaling and can inhibit complex IV, reducing oxygen consumption by mitochondria. Under low oxygen concentration nitrite $\left(\mathrm{NO}_{2}^{-}\right)$can be converted to $\mathrm{NO}$ by deoxygenated myoglobin (Mb), xanthine oxidoreductase (XO), complex III, and complex IV (ETC) allowing for complex IV inhibition. Nitrite can directly inhibit complex I via S-nitrosylation during ischemia resulting in suppressed oxidative stress leading to protection. 
ischemia. Overall, the S-nitrosylation on cysteine 39 within the ND3 subunit of complex I serves to decrease complex I generated ROS and preserve complex I activity.

Nitrite via conversion to NO has been shown to compete with oxygen for binding to the cytochrome $\mathrm{a}_{3}$ and $\mathrm{Cu}_{\mathrm{B}}$ binuclear complex and this inhibits complex IV activity leading to suppression of oxygen consumption (81). NO inhibition of complex IV is reversible and this inhibition is thought to be a protective mechanism by preserving oxygen levels in hypoxic tissue.

Other mitochondrial mechanisms of protection with nitrite include inhibition of MPT opening (73), mitochondrial fusion (84), and AMPK activation (82). The opening of the MPT allows the release of cytochrome $\mathrm{c}$ into the cytosol inducing activation of the intrinsic pathway of apoptosis and nitrite is associated with decreased apoptosis in IR injury. The exact mechanism of nitrite reducing MPT opening and cytochrome $\mathrm{c}$ release remains unknown, as this may occur through reduction in ROS (40), direct inhibition of cytochrome c release via nitrosylation (86) or modulation of MPT protein components (87). Nitrite activation of PKA mediates phosphorylation of dynamin related protein 1 (Drp1) leading to inactivation and an increase in mitochondrial fusion (84). Attenuation of increased Drp1 protein levels following $\mathrm{Ml}$ is associated with cardioprotection (88), suggesting that nitrite plays a role in mitochondrial dynamics. Nitrite induced mitochondrial fusion also leads to activation of AMPK leading to mitochondrial biogenesis (84) and AMPK activation is known to be cardioprotective (89). 
Overall, mitochondria appear to play a prominent role in protective effects of nitrite.

The numerous studies highlighting the cardioprotective effects of nitrite lead to two clinical trials to determine whether nitrite supplementation during acute $\mathrm{MI}$ in humans was protective $(90,91)$. Both clinical trials cited no protective effect of nitrite in acute $\mathrm{Ml}$, suggesting that nitrite-mediated cardioprotection is not applicable in humans. While the hypoxic environment allows for nitrite conversion to $\mathrm{NO}$, it also allows the formation of nitrogen species such as nitrogen dioxide and NO+. Additionally, NO can react with superoxide to form peroxynitrite. Nitrogen dioxide and peroxynitrite can react with thiols and tyrosines on proteins (92), altering protein function. Nitrogen dioxide and the nitrosonium ion can react with alkenes resulting in the formation of nitro-fatty acids (93). In this regard the cardioprotection afforded by nitrite may be influenced by the surrounding environment such as proteins or alkenes that can be post-translationally modified by secondary nitrogen species arising from nitrite.

\section{Conjugated Linoleic Acid}

\section{Sources and Structure}

Conjugated linoleic acid or CLA is an 18:2 unsaturated fatty acid and is an isomer of linoleic acid (Fig. 4). There are 28 different isomers of CLA with the cis- 
Figure 4. Cis-9 trans-11 isomer of conjugated linoleic acid

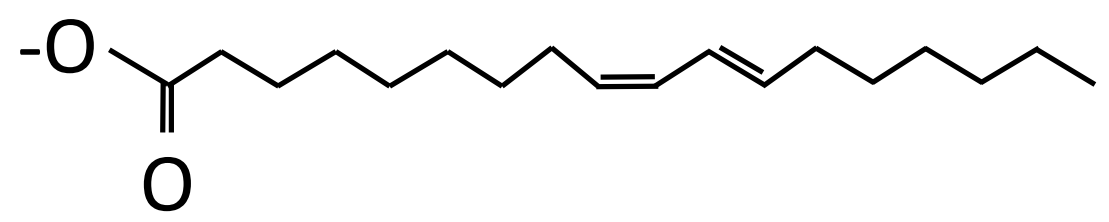

Figure 4. Cis-9 trans-11 isomer of conjugated linoleic acid. Conjugated linoleic acid is an 18:2 fatty acid with the cis-9 trans-11 isomer containing two double bonds at positions 9 and 11 . 
9 trans-11 and trans-10 cis-12 isomers comprising the majority of the CLA (94). CLA is produced in ruminant animals by two pathways: 1 . bacteria within the stomach that isomerizes linoleic acid to CLA and 2. delta-9-desaturase conversion of vaccenic acid (trans-11 18:1 fatty acid) to CLA. The delta-9desaturase pathway of cLA represents the majority source of CLA in cattle (95). cLA is found within a variety of foods including beef, pork, lamb, chicken, milk, butter, cheese, and vegetable oils (96). The total amount of cLA in beef is approximately $1 \%$ of fat and $\mathrm{CLA}$ content can increase by supplementing forage and oils to cattle (97). The majority of total CLA within dietary food products is composed of the cis- 9 trans- 11 isomer of cLA representing approximately $90 \%$ of total CLA. The estimate of dietary intake of CLA within adults in the United States is $0.2 \mathrm{~g} /$ day (98), with dietary habits such as higher intake of ruminant fat influencing individual dietary intake of CLA. CLA levels can also be increased by cLA supplementation, as CLA is commercially sold as a weight loss supplement that is unregulated by the Food and Drug Administration (FDA) (99). The FDA also excludes CLA from nutrition labeling regulations requiring levels of trans fats in food to be disclosed, as CLA is labeled as a natural trans-fat (99). As trans fats from hydrogenated oil are linked to increased cardiovascular risk factors, efforts have been made to understand the role of CLA in cardiovascular health and disease.

\section{Biological Role of cLA}


Conjugated linoleic acid has been evaluated in numerous epidemiological studies assessing the risk of cardiovascular disease or MI $(100,101)$. CLA displayed neither a positive nor a negative correlation for development of $\mathrm{MI}$ or cardiovascular disease, with exception of one study in which adipose cis-9 trans$11 \mathrm{cLA}$ was associated with lower risk of MI (102). CLA can act as a peroxisome proliferator-activated receptors (PPARs) agonist and PPAR activation leads to gene transcription involved in lipid metabolism, insulin signaling and inflammation (103). The effect of CLA on weight loss in animal models has translated over to humans as CLA is commercially sold as a weight loss supplement. However, the FDA does not regulate CLA. The target patient population for CLA supplementation is obese individuals setting to lose weight.

In clinical trials of obese individuals CLA had no effect on lipid profiles and the cis-9 trans-11 isomer of CLA increased 8-iso-prostaglandin F2 $\alpha$ (8-isoPGF2 $\alpha$ ) levels in urine (104). 8-iso-PGF2 $\alpha$ is a marker of lipid peroxidation suggesting that CLA increases lipid peroxidation in obese individuals. CLA has been shown to increase ROS production in macrophages (105) and promote oxidative stress in cardiac tissue of male Wistar rats (106), potentially explaining the increased 8-iso-PGF2 $\alpha$ in obese individuals. The cis-9 trans-11 isomer of cLA has been shown to uncouple eNOS and increase NADPH oxidase 2 (Nox2) protein expression in endothelial cells and murine cardiac tissue (107). This uncoupling of eNOS and increased Nox2 expression could lead to an increase in oxidative stress. In contrast to these results, CLA has also been shown to be beneficial. Healthy male individuals given 3 doses of cis- 9 trans-11 cLA displayed 
a significantly reduced total cholesterol to high density lipoprotein cholesterol (TC:HDL-C) ratio (108). The most significant beneficial effect of $C L A$ in relation to cardiovascular disease is the reduction in atherosclerosis and total cholesterol (109-111). The cis-9 trans-11 isomer of cLA decreased atherosclerotic lesions in the aorta (109), reduced total cholesterol (110) and cholesterol accumulation within the aorta (111).

Two potential pathways for cLA's mechanism of action have been described: 1. activation of PPARs and 2. inhibition of cyclooxygenase (COX), which is responsible for production of prostaglandins and thromboxane necessary for inflammation and vascularization (94). The activation of PPARa by cLA (112) leads to gene transcription of several genes involved in beta oxidation and increased mitochondrial biogenesis (113). The activation of PPARa links the ability of cLA to modulate lipid metabolism but PPAR $\alpha$ knockout mice displayed similar lipid metabolism as wild type mice supplied with CLA (114), suggesting cLA mediates changes in lipid metabolism independent of PPARa (114).

Mitochondria play a central role in lipid metabolism as beta oxidation of fatty acids occurs within mitochondria and CLA has been shown to mediate changes at the level of mitochondria. CLA has been shown to inhibit the beta oxidation of other fatty acids within the liver (115), increase activity of carnitine palmitoyltransferase I (CPTI) (116) and increase activity of the mitochondrial citrate carrier (116). These results suggest $C L A$ is able to directly modulate mitochondrial function; however the impact of CLA on cardiac mitochondrial function is unknown. 
The literature on CLA suggests a wide array of biological effects including beneficial, detrimental or no effect (101). This can be explained by dose and duration of CLA administration. Most animal studies using CLA give super physiological doses of CLA and the duration of CLA ranges from days to weeks (101). The composition of the cLA dose is important to consider as well, and the majority of studies have used an isomeric mixture of CLA composed of both cis-9 trans-11 and trans-10 cis- 12 . This is an important characteristic of study design as these isomers can have differing effects on study end points (101). Due to the high abundance of the cis-9 trans-11 isomer of CLA within the diet it is important to study the isomeric specific effects. The cis-9 trans- 11 isomer of CLA decreased cardiac function in aged mice and co-administration of nitrite reversed this cardiac dysfunction (107). The interaction of $C L A$ and nitrite brings up an overlooked mechanism of action of $\operatorname{cLA}$; the nitration of $\operatorname{cLA}$. Due to the conjugated double bond nature of CLA, the cis-9 trans-11 isomer is the preferred endogenous substrate of fatty acid nitration (117).

\section{Nitrated Fatty Acids}

\section{Mechanisms of Formation}

The formation of nitrated fatty acids is induced by NO derived species reacting via multiple mechanisms, ultimately resulting in the addition of nitrogen dioxide to a double bond. Peroxynitrite, oxidation of nitrite, protonation of nitrite, and aerobic reactions of $\mathrm{NO}(93)$ are all mechanisms that can mediate nitration 
of fatty acids; thereby depicting a complex interaction between NO, NO derived oxides of nitrogen, peroxynitrite, and oxygen derived inflammatory mediators (ex. superoxide) leading to nitrated fatty acids. Fatty acids such as linoleic and oleic acid are targets of nitration due to their high abundance and double bond structure lending to nitrogen dioxide addition via either homolytic or hetrolytic reactions (93). The formation of nitrated fatty acids is influenced by microenvironment including: aqueous vs hydrophobic environment, flux of nitrating and oxidizing species, antioxidants, and fatty acid beta-oxidation rates (118). Nitrated fatty acids exist in an equilibrium between the nitroalkene and nitrohydroxy form as nitro-fatty acids react under hydroxide anions within water (93). Nitroalkenes are stable in free or esterified forms under hydrophobic

conditions such as lipid membranes or lipoproteins and once released to aqueous environments can either release NO, bind and activate PPARs, or posttranslational modify proteins (118).

\section{Biological Role of Nitrated Fatty Acids}

Nitroalkenes decay in aqueous solutions leading to the release of $\mathrm{NO}$; however, under physiologically conditions the release of NO by nitrated fatty acids is less than one percent (93). Nitroalkenes have been shown to activate PPAR gamma leading to decreased insulin and glucose levels in leptin deficient mice (119) and elevated nitrated fatty acids are found in the plasma of postprandial and hyperlipidemic humans (120). The activation of PPAR gamma by nitro-fatty acids was similar to the diabetic drug rosiglitazone without the 
increased adipogenesis suggesting a unique mechanism of action of PPAR gamma by nitro-fatty acids (121). A more important and diverse role of nitrated fatty acids is in inflammation.

Due to the electrophilic nature of nitrated fatty acids, they can react with protein thiols and histidine residues, thereby modulating cell signaling (93). Kelch-like $\mathrm{ECH}$-associating protein 1 (KEAP1) is a cytoplasmic repressor protein of Nrf2 which holds Nrf2 in the cytoplasm in an inactive state. KEAP1 contains a reactive cysteine that undergoes electrophilic modification by nitrated fatty acids (122) allowing Nrf2 to translocate to the nucleus and upregulate the transcription of phase II antioxidant enzymes. Heme-oxygenase-1 (HO-1) is one of these phase II antioxidant enzymes and nitrofatty acids have been shown to increase HO-1 protein expression (123). Nitrated fatty acids can also nitroalkylate p65, a component of the NF-kB inflammatory signaling pathway, thereby inhibiting p65 DNA binding (124) and decreasing pro-inflammatory cytokine gene expression.

Due to the increase in nitrative and oxidative stress associated with $\mathrm{Ml}$, the formation of nitrated fatty acids increases following ischemia reperfusion injury (125). The exogenous administration of a nitrated oleic acid prior to ischemia reperfusion injury reduced infarct size (47), showing endogenous generation or exogenous administration of nitrated fatty acids is cardioprotective. Research into the mechanisms by which nitroalkenes mediate cardioprotection highlighted a mitochondrial mechanism as nitroalkenes promote mitochondrial uncoupling (47) and nitroalkylation of ANT-1 (46). Importantly, mitochondrial targeted nitroalkenes are more protective than non-mitochondrial nitroalkenes 
(126). Recent evidence has shown that CLA is the preferred endogenous substrate for fatty acid nitration, generating nitro-cLA $(117,127)$ and a combination of CLA and nitrite is protective against cardiac injury (88). Due to the highly dependent role of mitochondria on nitro-fatty acid cardioprotection, it remains unknown if precursors to fatty acid nitration (CLA and nitrite) modulate mitochondrial function under baseline and cardiac injury settings.

\section{Hypothesis and Research Strategy}

It is clear that $\operatorname{cLA}$, nitrite and mitochondria play important roles in cardiovascular health and disease; therefore it is important to investigate the effect of cLA and nitrite consumption on mitochondrial function. To evaluate the impact of CLA and nitrite on cardiac mitochondrial function the following hypothesis was proposed: Co-treatment of $C L A$ and nitrite protects against cardiac injury and age related cardiac dysfunction via beneficially altering mitochondrial function.

In this study, cardiac mitochondrial function was examined under baseline and cardiac injury conditions following $\mathrm{CLA}$, nitrite and the combination treatment of CLA and nitrite. We first examined the impact on cardiac mitochondria following a ten day treatment with $\mathrm{CLA}$, nitrite and co-treatment with $\mathrm{CLA}$ and nitrite under baseline conditions. This was followed up by examining the impact of $\mathrm{Ml}$ and aging on cardiac mitochondrial function and how treatment with cLA, nitrite and co-treatment with CLA and nitrite mediated changes in cardiac mitochondrial function following $\mathrm{Ml}$ and aging. 
Our results demonstrate that co-treatment with CLA and nitrite decreases mitochondrial state 3 respiration and complex III activity. In addition, we found that $\mathrm{MI}$ increases mitochondrial state 3 respiration, complex III activity, and hydrogen peroxide generation. However, the co-treatment of $C L A$ and nitrite was able to attenuate these changes, potentially contributing to cardioprotection. These results reveal that in vivo supplementation of $C L A$ and nitrite is able to modulate mitochondrial function and provides an explanation for $C L A$ and nitrite cardioprotection. 


\section{CHAPTER II \\ MATERIAL AND METHODS}

\section{Experimental Procedures}

\section{Animals}

C57BL/6J mice were obtained from Jackson Laboratory and all animal studies were approved by the University of Louisville Institutional Animal Care and Use Committee. Mice were fed standard chow and water ad libitum.

\section{Experimental Design \\ Chapter III}

C57BL/6J mice divided into four groups: control, CLA, nitrite, and $\mathrm{cLA} /$ nitrite. cLA was given via osmotic mini-pump (20mg/kg/d) and sodium nitrite (50ppm) was supplemented in drinking water for 10 days.

\section{Chapter IV}

C57BL/6J mice were divided into eight groups: control, CLA, nitrite, CLA/nitrite, MI- control, MI- CLA, MI- nitrite, MI- CLA/nitrite. CLA was given via osmotic mini-pump (20mg/kg/d for non-Ml and $10 \mathrm{mg} / \mathrm{kg} / \mathrm{d}$ for $\mathrm{Ml}$ ) and sodium nitrite (50ppm) was supplemented in drinking water for 3 days prior to $\mathrm{Ml}$ and continued for 10 days after Ml.

\section{Chapter V}


C57BL/6J mice were aged to 10 or 33 month prior to treatment. Mice were divided into four groups: control, CLA, nitrite, and CLA/ nitrite. CLA was given via osmotic mini-pump (20mg/kg/d) and sodium nitrite (50ppm) was supplemented in drinking water for 6 weeks.

\section{Blood Glucose Measurements}

Mice were fasted overnight prior to blood glucose measurements from tail blood using the TRUEresult (NIPRO Diagnostics) blood glucose monitor. Measurements were taken prior and following treatment for control, CLA, nitrite and $\mathrm{CLA} /$ nitrite.

\section{Mouse Model of Myocardial Infarction}

Male C57BL/6J mice, 10- to 12-wks old, were anesthetized with isoflurane, intubated and ventilated with CWE advanced ventilator (Webster,TX). Body temperature was maintained with an Indus Temperature feedback/surgical table and ECG system. Aseptic procedure was used for preparation of the surgical site through scrubbing with a $0.8 \%$ chlorhexidine solution. A left thoracotomy was performed via the fourth intercostal space and the lungs retracted to expose the heart. After opening the pericardium, the left anterior descending (LAD) coronary artery was ligated with an 8-0 silk suture near its origin between the pulmonary outflow tract and the edge of the atrium. Ligation was deemed successful when the anterior wall of the left ventricle (LV) became pale in color. The lungs were inflated by increasing positive end-expiratory 
pressure, and the thoracotomy side was closed in layers. The lungs were reexpanded, and the chest was closed. The animals were removed from the ventilator and allowed to recover on a heating pad. Mice were checked daily for signs of pain or distress and buprenorphine at $0.05 \mathrm{mg} / \mathrm{kg} \mathrm{SQ}$ is given before and every $12 \mathrm{~h}$ for 48 hours.

\section{Heart Mitochondria Isolation}

Hearts were excised and placed immediately in ice cold isolation media 1 (250mM sucrose, $0.5 \mathrm{mM} \mathrm{Na}_{2}$ EDTA, and $10 \mathrm{mM}$ Tris, $\mathrm{pH}$ 7.4). Hearts were minced, washed 3 times and homogenized at 40 RPM using the GKHHomogenizer (Glas-Col). Heart homogenate was centrifuged at $1000 \times \mathrm{g}$ for 3 minutes at $4^{\circ} \mathrm{C}$ and supernatant was filtered through gauze sponge into a new tube followed by centrifugation at $10,000 \times \mathrm{g}$ for 10 minutes. The mitochondrial pellet was washed using isolation media $2\left(250 \mathrm{mM}\right.$ sucrose, $0.5 \mathrm{mM} \mathrm{Na} \mathrm{N}_{2}$ EDTA, $10 \mathrm{mM}$ Tris, and 1g/L BSA, $\mathrm{pH} 7.4$ ) and centrifuged at $10,000 \times \mathrm{g}$ for 10 minutes. The mitochondrial pellet was washed again with isolation media 2 and centrifuged at $10,000 \times \mathrm{g}$ for 10 minutes. Following centrifugation the mitochondrial pellet was dissolved in respiration media (0.5mM EGTA, 3mM $\mathrm{MgCl}_{2}, 60 \mathrm{mM}$ K-lactobionate, $20 \mathrm{mM}$ taurine, $10 \mathrm{mM} \mathrm{KH}_{2} \mathrm{PO}_{4}, 20 \mathrm{mM}$ HEPES, $110 \mathrm{mM}$ Sucrose, and 1 $\mathrm{g} / \mathrm{L}$ BSA). Heart mitochondria concentration was determined using the Bradford Protein Assay and was used immediately for respiration or stored at $-80^{\circ} \mathrm{C}$ for complex activity or western blot. 


\section{Respiration}

Mitochondria $(0.025 \mathrm{mg} / \mathrm{ml})$ was added to closed Oxygraph-2k (Oroboros Instruments) chambers containing $2 \mathrm{ml}$ of respiration buffer (0.5mM EGTA, 3mM $\mathrm{MgCl}_{2}, 60 \mathrm{mM}$ K-lactobionate, $20 \mathrm{mM}$ taurine, $10 \mathrm{mM} \mathrm{KH}_{2} \mathrm{PO}_{4}, 20 \mathrm{mM}$ HEPES, 110mM Sucrose, and 1 g/L BSA). LEAK (proton leak) respiration was measured following addition of $10 \mathrm{mM}$ glutamate and $2 \mathrm{mM}$ malate, followed by addition of $1 \mathrm{mM}$ ADP for measurement of State 3 respiration. Cytochrome c $(2.5 \mu \mathrm{M})$ was added following induction of State 3 respiration as a control to ensure maximal respiration in State 3 and evaluate integrity of the outer mitochondrial membrane. Oligomycin $\mathrm{A}(2.5 \mu \mathrm{M})$ was then added to induce State 4 respiration, followed by addition of $3.5 \mu \mathrm{M}$ carbonyl cyanide p-trifluoro-methoxyphenyl hydrazone (FCCP) to obtain maximum mitochondrial respiration in the non-coupled state of electron transfer system capacity. Upon depletion of oxygen $0.5 \mu \mathrm{M}$ antimycin $\mathrm{A}$, a complex III inhibitor, was added to the chamber followed by re-oxygenation to measure residual oxygen consumption due to oxidative side reactions. Figure 5 shows representative Oxygraph-2k graph following respiration protocol.

\section{Complex I Activity Assay}

Complex I activity assay was adapted from previously described method (128) with slight modification. Briefly, mitochondria were diluted to concentration of $0.2 \mu \mathrm{g} / \mu \mathrm{l}$ and sonicated (QSonica) $4 x$ for 5 seconds. $12 \mu \mathrm{g}$ of mitochondria was added to reaction mixture $(25 \mathrm{mM}$ potassium phosphate $\mathrm{pH} 7.4,80 \mu \mathrm{M} \mathrm{NADH}$, $2 \mathrm{mM} \mathrm{KCN}, 2 \mu \mathrm{M}$ antimycin $\mathrm{A}, 60 \mu \mathrm{M}$ decyclubiquinone, and $3 \mathrm{mg} / \mathrm{mL} \mathrm{BSA}$ ) in 1.5 
$\mathrm{mL}$ cuvette (Starna Cells) and change in absorbance was read at $340 \mathrm{~nm}$ for 4 minutes using Synergy 2 (BioTek) spectrophotometer. Rotenone $(4 \mu \mathrm{M})$ was added to reaction mixture to determine rotenone insensitive complex I activity and subtracted from total activity to determine rotenone specific complex I activity.

\section{Complex II Activity Assay}

Complex II activity assay was adapted from a previously described method (129) with slight modification. Briefly, $5 \mu \mathrm{g}$ mitochondria was added to reaction mixture $(25 \mathrm{mM}$ potassium phosphate $\mathrm{pH} 7.4,50 \mu \mathrm{M}$ decyclubiquinone, $80 \mu \mathrm{M}$ 2,6-Dichlorophenolindophenol sodium salt hydrate, $20 \mathrm{mM}$ succinate, $1 \mathrm{mg} / \mathrm{mL}$ BSA and $300 \mu \mathrm{M} \mathrm{KCN}$ ) in $1.5 \mathrm{~mL}$ cuvette (Starna Cells) and incubated for 10 minutes. Decyclubiquinone $(50 \mu \mathrm{M})$ was added to start reaction and change in absorbance was monitored at 600nm for 4 minutes using Synergy 2 (BioTek) spectrophotometer. Malonate $(10 \mathrm{mM})$ was added to reaction mixture to determine malonate insensitive complex $\mathrm{II}$ activity and subtracted from total activity to determine malonate specific complex II activity.

\section{Complex III Activity Assay}

Complex III activity assay was adapted from a previously described method (129) with slight modification. Briefly, $5 \mu \mathrm{g}$ mitochondria was added to reaction mixture $(25 \mathrm{mM}$ potassium phosphate $\mathrm{pH} 7.4,100 \mu \mathrm{M}$ decyclubiquinol, $75 \mu \mathrm{M}$ cytochrome c, $500 \mu \mathrm{M} \mathrm{KCN}$, and $100 \mu \mathrm{M} \mathrm{Na}{ }_{2} \mathrm{EDTA}$ ) in $1.5 \mathrm{~mL}$ cuvette and 
change in absorbance was read at 550nm for 4 minutes using Synergy 2 (BioTek) spectrophotometer. Antimycin A $(10 \mu \mathrm{g} / \mathrm{mL})$ was added to reaction mixture to determine antimycin $A$ insensitive activity and subtracted from total activity to determine antimycin A specific complex III activity.

\section{Complex IV Activity Assay}

Complex IV activity assay was adapted from a previously described method (129) with slight modification. Briefly, $5 \mu \mathrm{g}$ mitochondria was added to reaction mixture $(50 \mathrm{mM}$ potassium phosphate $\mathrm{pH} 7.4$, and $30 \mu \mathrm{M}$ reduced cytochrome c) in $1.5 \mathrm{~mL}$ cuvette (Starna Cells) and change in absorbance was monitored at 550nm for 4 minutes using Synergy 2 (BioTek) spectrophotometer. $\mathrm{KCN}(300 \mu \mathrm{M})$ was added to reaction mixture to determine $\mathrm{KCN}$ insensitive activity and subtracted from total activity to determine KCN specific complex IV activity.

\section{Western Blot Analysis}

Heart tissue was snap frozen in liquid nitrogen and isolated mitochondria were stored at $-80^{\circ} \mathrm{C}$ until needed. Heart tissue was homogenized and protein concentration determined using Bradford Protein Assay. Heart tissue or isolated mitochondria samples were then sonicated $2 x$ for 5 seconds and subjected to SDS-PAGE method. MitoProfile® Total OXPHOS Rodent WB Antibody Cocktail $(1.5 \mu \mathrm{g} / \mathrm{mL})$ was purchased from ABCAM for NADH dehydrogenase ubiquinone 1 beta subcomplex 8 (NDUFB8), succinate dehydrogenase complex subunit B 
(SDHB), ubiquinol-cytochrome c reductase core protein II (UQCRC2), mitochondrially encoded cytochrome c oxidase 1 (MTCO1) and ATP synthase subunit alpha (ATP5A).

\section{$\mathrm{H}_{2} \mathrm{O}_{2}$ Assay}

$25 \mu \mathrm{g}$ of heart tissue supernatant was incubated in phosphate buffered saline (PBS) $\mathrm{pH} 7.4,49 \mu \mathrm{M}$ Amplex® UltraRed (Invitrogen), and $0.1 \mathrm{U} / \mathrm{mL}$ horseradish peroxidase (Simga) for 30 minutes. Absorbance was measured at $560 \mathrm{~nm}$ and hydrogen peroxide concentration was calculated using hydrogen peroxide standard curve.

\section{Statistical Analysis}

Data are presented as mean \pm SEM. One way ANOVA with Bonferroni post hoc test, two way ANOVA with Bonferroni post hoc test or unpaired Student's t-test were performed to determine significance using GraphPad Prism 6 software. 


\section{CHAPTER III}

\section{COMBINATION TREATMENT WITH CLA AND NITRITE IN VIVO CHANGES MITOCHONDRIAL FUNCTION}

\section{Chapter Overview}

Nitrite and CLA are dietary components found in green leafy vegetables and runimant meat respectively. CLA and nitrite are reported to have overall benefits on cardiovascular health and studies have shown that either nitrite or CLA can mediate changes in the activity of mitochondrial proteins and influence mitochondrial metabolism. However, it is not known how a combination of both cLA and nitrite impacts cardiac mitochondrial function. Cardiac mitochondria are essential for maintaining normal cardiac function and the impact of dietary cLA and nitrite on cardiac mitochondrial function may have a profound impact on overall cardiac health and function. In this study we examined how CLA, nitrite and a combination treatment of $\mathrm{CLA}$ and nitrite impacted cardiac mitochondrial function and individual electron transport chain activity. This study is the first to show that nitrite and CLA impact cardiac mitochondrial function in vivo. 


\section{Introduction}

Conjugated linoleic acid ( $C L A)$ is an 18:2 unsaturated fatty acid and an isomer of linoleic acid. CLA is found within ruminant meat and dairy products with the majority of dietary $\mathrm{CLA}$, approximately $72-94 \%$, composed of the cis- 9 trans11 isomer. The dietary intake of CLA within the United States is approximately $0.2 \mathrm{~g} /$ day (98) and intake can increase as CLA is commercially sold as a weight loss supplement. cLA plays a role in lipid metabolism and glucose homeostasis (130) via activation of peroxisome proliferator activated receptor (PPAR) activation (131). cLA can also directly mediate effects on mitochondria via inhibition of fatty acid beta oxidation (115), increased activity of carnitine palmitoyltransferase I activity (116) and mitochondrial citrate carrier protein (116). The role of the cis-9 trans-11 isomer of CLA on cardiac mitochondrial function is unknown at this time.

Nitrite $\left(\mathrm{NO}_{2}{ }^{-}\right)$is a dietary constituent found within spinach and beet juice. Dietary nitrite represents an endogenous reservoir of nitric oxide (NO), as nitrite can be reduced to NO (62). NO plays an important role in cardiovascular health due to its role in regulating vascular tone (62) via activation of soluble guanylyl cyclase. More recent evidence suggests nitrite is cardioprotective via mitochondrial dependent mechanisms including inhibition of mitochondrial pore transition opening (73), mitochondrial fusion (84) and post-translational modifications on complex I (40). Nitrite has also been shown to mediate changes upstream of mitochondria including activation of protein kinase $A$ (PKA) which phosphorylates and inactivates dynamin related protein 1 (Drp1) leading to 
increased mitochondrial fusion (84). Nitrite also serves to activate adenine monophosphate kinase (AMPK), (82) a metabolic sensor involved in modulating metabolism. Due to the dietary nature of nitrite and its effects on mitochondria it is important to assess how nitrite influences cardiac mitochondrial function.

Recent evidence from our lab reported that CLA induced cardiac dysfunction in aged mice that was rescued with nitrite supplementation (107) and combination treatment of $\mathrm{CLA}$ and nitrite is protective in a murine model of myocardial infarction (MI) (88). Cardiac mitochondria play an essential role in cardiac function and this study investigates how CLA and nitrite affects cardiac mitochondrial function in vivo. 


\section{Results}

To test the hypothesis that co-treatment of CLA and nitrite would impact mitochondrial function, mitochondrial respiration (as outlined in Fig. 5) was assessed in cardiac mitochondria isolated from $\mathrm{C} 57 \mathrm{BL} / 6 \mathrm{~J}$ mice treated with $\mathrm{cLA}$, nitrite and co-treatment. Weight measurements (Fig. 6A), demonstrated animals remained healthy with given does of CLA and nitrite. Blood glucose (Fig. 6B) was also monitored, as cLA (132) and nitrated fatty acids (93) can impact blood glucose homeostasis, and was unaltered following all treatment groups. Assessment of cardiac mitochondrial function demonstrated that state 3 respiration was significantly decreased following $C L A$ and co-treatment with $C L A$ and nitrite (Fig. 7B), while LEAK respiration (Fig. 7A), state 4 respiration (Fig. 7C) and ETS respiration (Fig. 7D) remained unchanged. The decrease in state 3 respiration was reflected in the respiratory control ratio (RCR) of CLA and cotreated mice (Fig. 7E) as the RCR was significantly decreased.

To determine if changes in mitochondrial respiration were due to changes in electron transport chain enzymatic activity, complex I,II, III and IV enzymatic activity assays were performed. Nitrite and the co-treatment of CLA and nitrite increased complex I activity (Fig. 8A) compared to control, while the combination treatment of CLA and nitrite decreased complex II (Fig. 8B) and III (Fig. 8C) activity compared to control. Complex II activity was also decreased with cLA treatment alone (Fig. 8B). Complex IV activity (Fig. 8D) showed no change with any treatment group and the ten day treatment of $c L A$, nitrite and co-treatment did not alter mitochondrial respiratory chain protein subunits (Fig. 9). 
Figure 5. Mitochondrial Respiration Protocol.

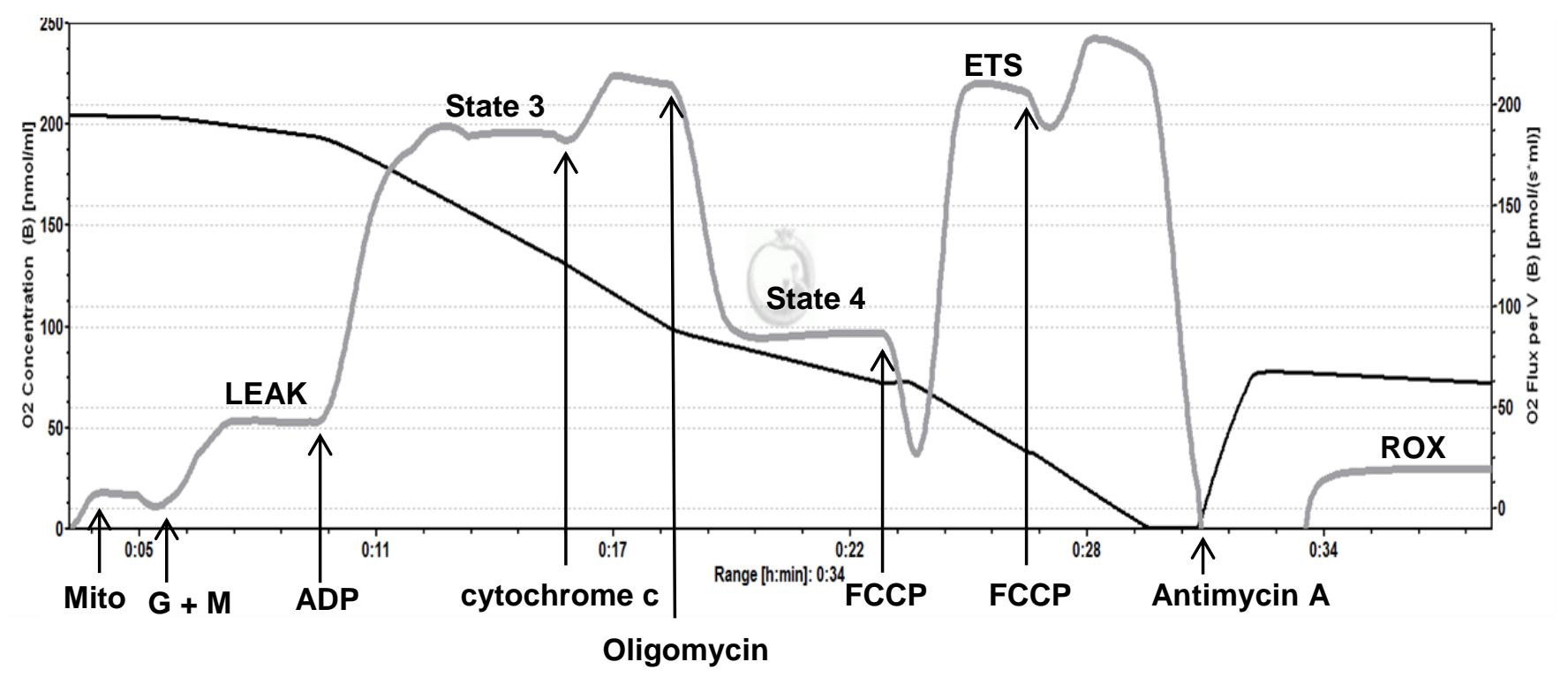

Figure 5. Mitochondrial Respiration Protocol. Simultaneous changes in oxygen concentration (black line, left y-axis) and oxygen flux per $\mathrm{V}$ (gray line, right $y$-axis) is monitored over time following substrate addition $(G+M$; glutamate and malate) where indicated. Mitochondrial LEAK (proton leak), State 3, State 4, ETS (electron transfer system) and ROX (residual oxygen consumption) respiration are obtained following substrate addition. 
Figure 6. Treatment with cLA, nitrite or combination does not alter animal weight and blood glucose.
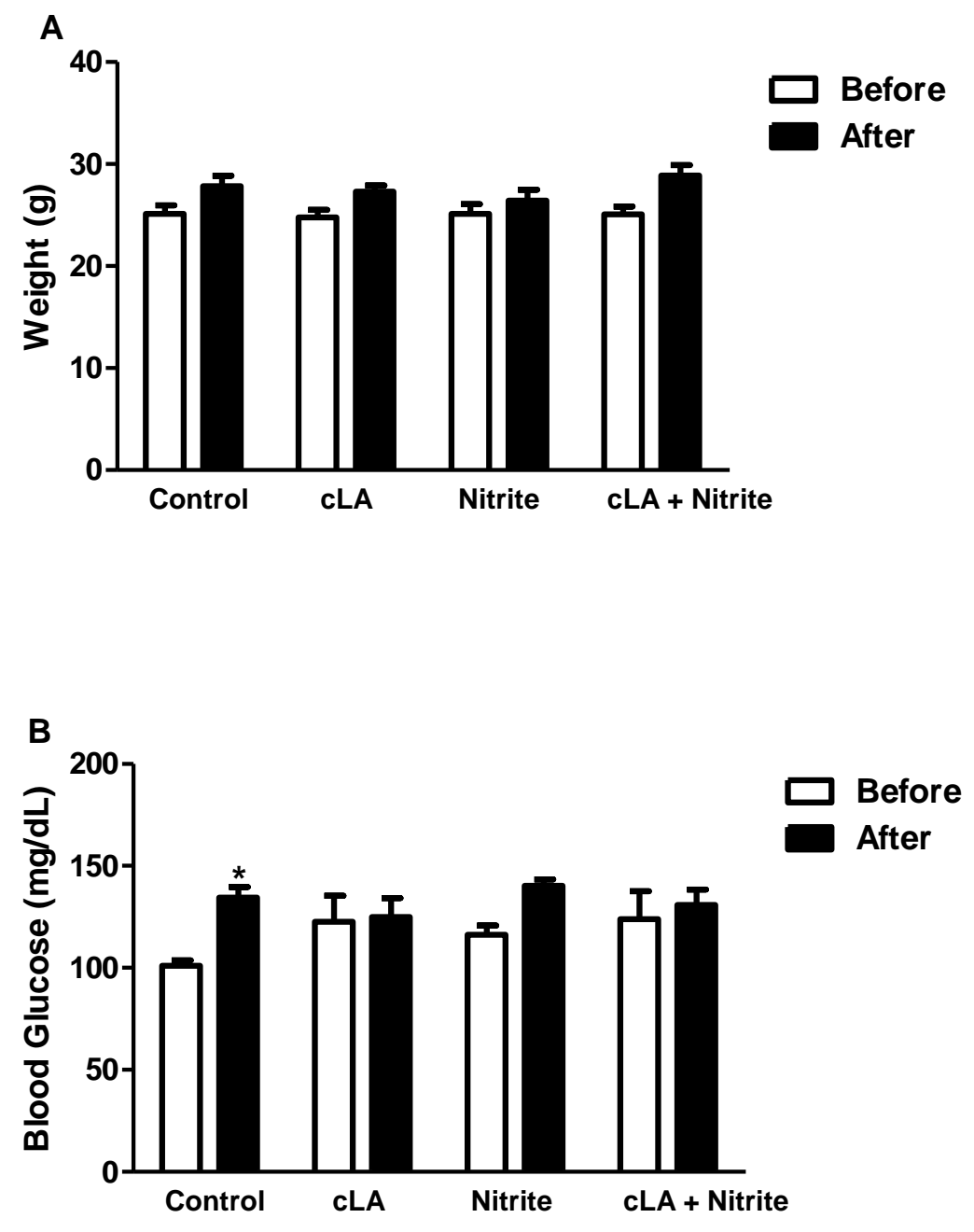

Figure 6. Treatment with cLA, nitrite or in combination does not alter animal weight and blood glucose. Weight $(A)$ and blood glucose $(B)$ were monitored and remained unchanged following 10 day treatment with $\mathrm{CLA}$, nitrite and co-treatment with $\operatorname{cLA}$ and nitrite. $\left({ }^{*} \mathrm{p}<0.05\right.$, control before vs control after, Two way ANOVA, $n=4$ for all groups.) 
Figure 7. State 3 respiration decreases following cLA treatment.
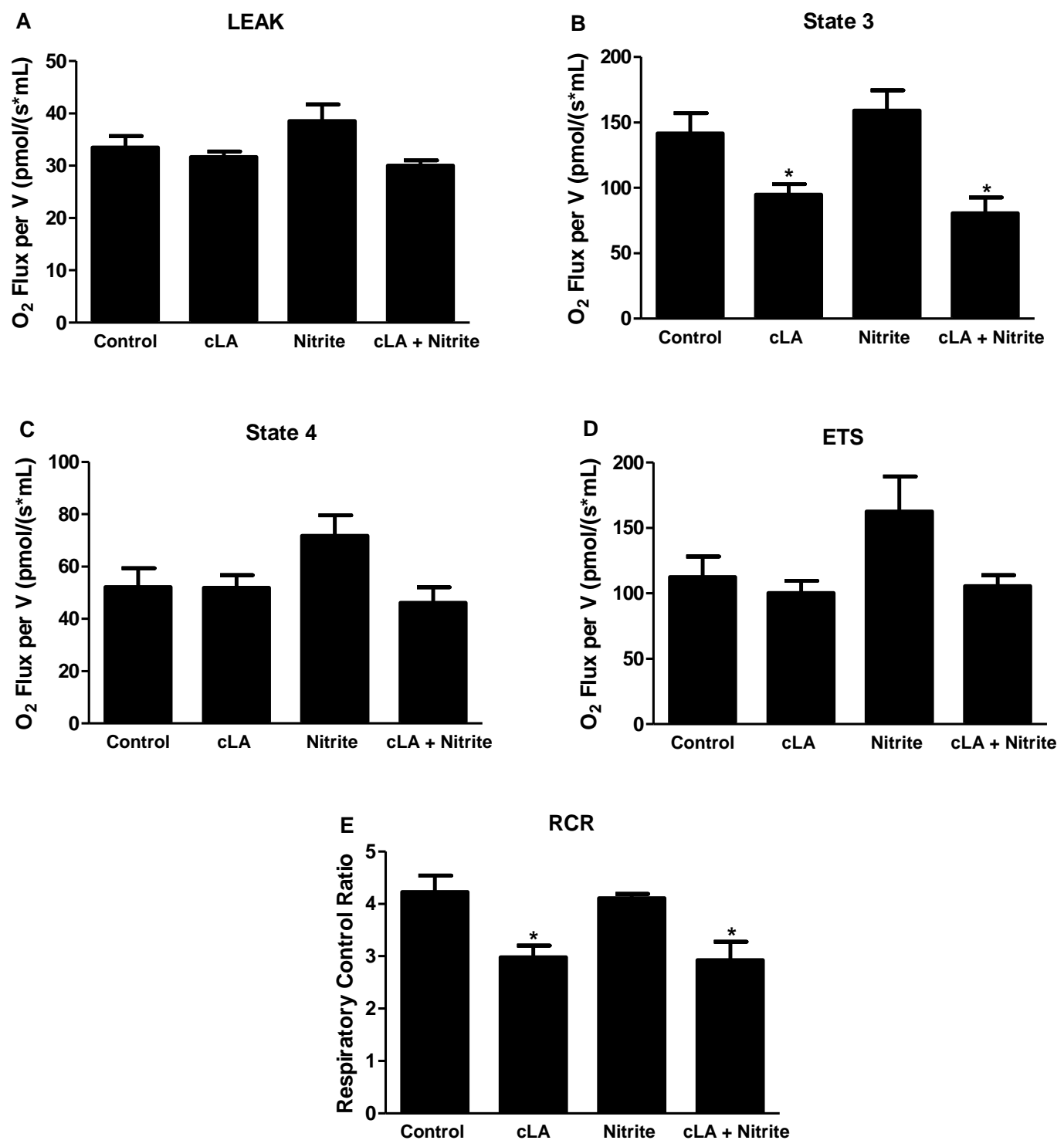

Figure 7. State 3 respiration decreases following cLA treatment. Ten day CLA and CLA + nitrite treatment decreases State 3 respiration (B) and the RCR (E) in cardiac mitochondria compared to control, while LEAK respiration (A), State 4 respiration (C) and ETS respiration (D) remain unchanged $\left({ }^{*} \mathrm{p}<0.05\right.$ compared to control, One way ANOVA. $n=4$ for control, $C L A$, and nitrite, $n=8$ for $\mathrm{cLA}+$ nitrite). 
Figure 8. Co-treatment with cLA and nitrite decreases complex II and III activity, while increasing complex I activity.
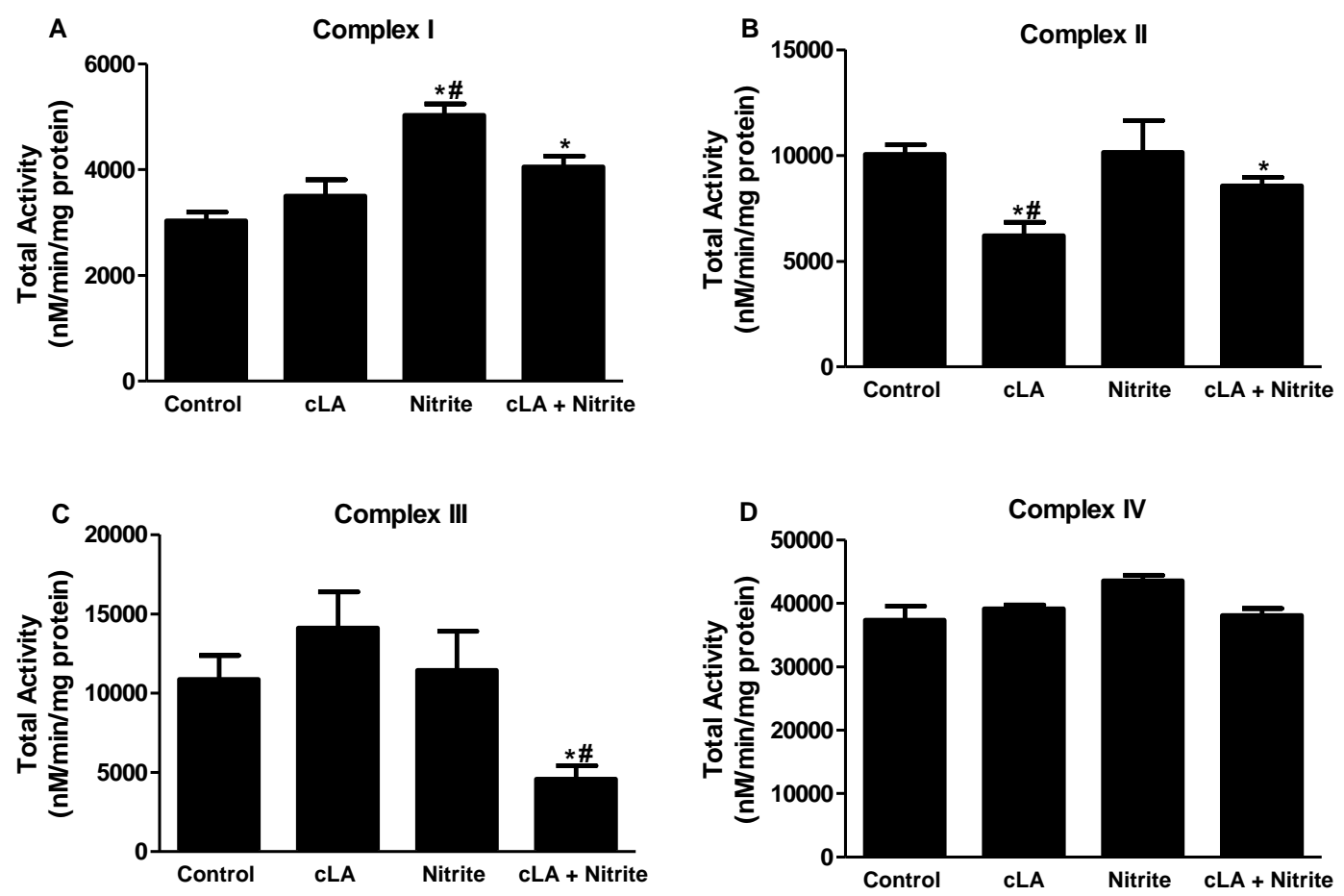

Figure 8. Co-treatment with CLA and nitrite decreases complex II and III activity, while increasing complex I activity. Complex I (A): $c L A+$ nitrite and nitrite increase complex I activity $\left({ }^{*} p<0.05\right.$ compared to control, $\# p<0.05$ compared to $\mathrm{CLA}$ and $\mathrm{CLA}+$ nitrite, One way ANOVA, $\mathrm{n}=8$ for control and $\mathrm{CLA}+$ nitrite, $n=7$ for $c L A$ and nitrite). Complex II (B): $c L A+$ nitrite and $c L A$ decrease complex II activity $\left({ }^{*} \mathrm{p}<0.05\right.$ compared to control, $\# p<0.05$ compared to nitrite, $\mathrm{cLA}+$ nitrite, One way ANOVA, $n=4$ for all groups). Complex III (C): $\mathrm{CLA}+$ nitrite decreases complex III activity $\left({ }^{*} p<0.05\right.$ compared to control, $\# p<0.05$ compared to cLA and nitrite, unpaired student t-test, $n=7$ for control, $n=6$ for $\operatorname{cLA}$ and $n=4$ for nitrite and CLA + nitrite). Complex IV (D): Complex IV activity remains unchanged in all treatment groups ( $\mathrm{n}=4$ for all groups). 
Figure 9. Mitochondrial respiratory chain protein subunit levels remain unchanged following cLA, nitrite or combination treatment with cLA and nitrite.

A

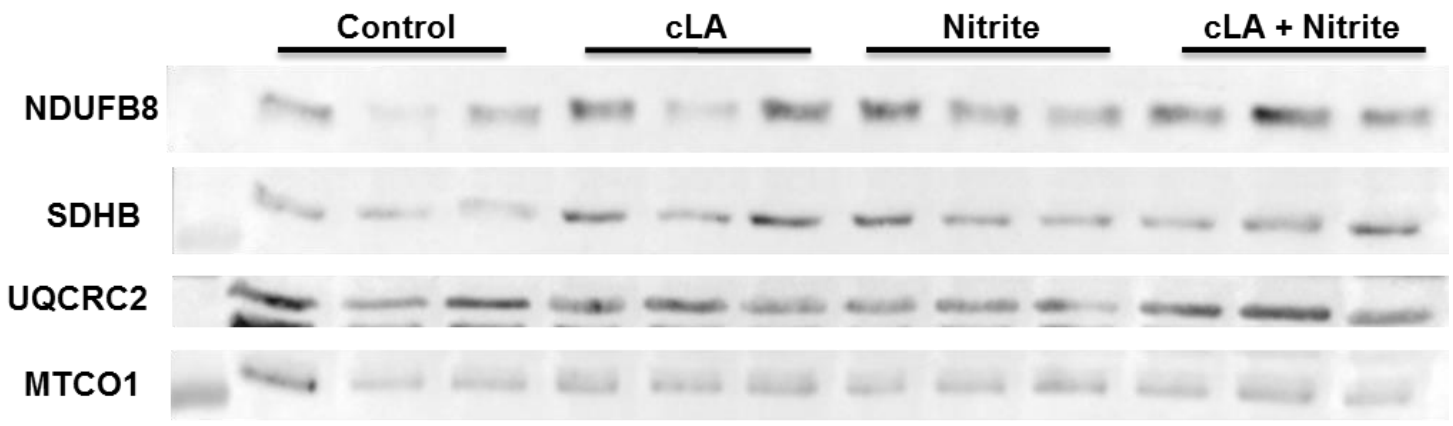

ATP5A

Ponceau

Stain
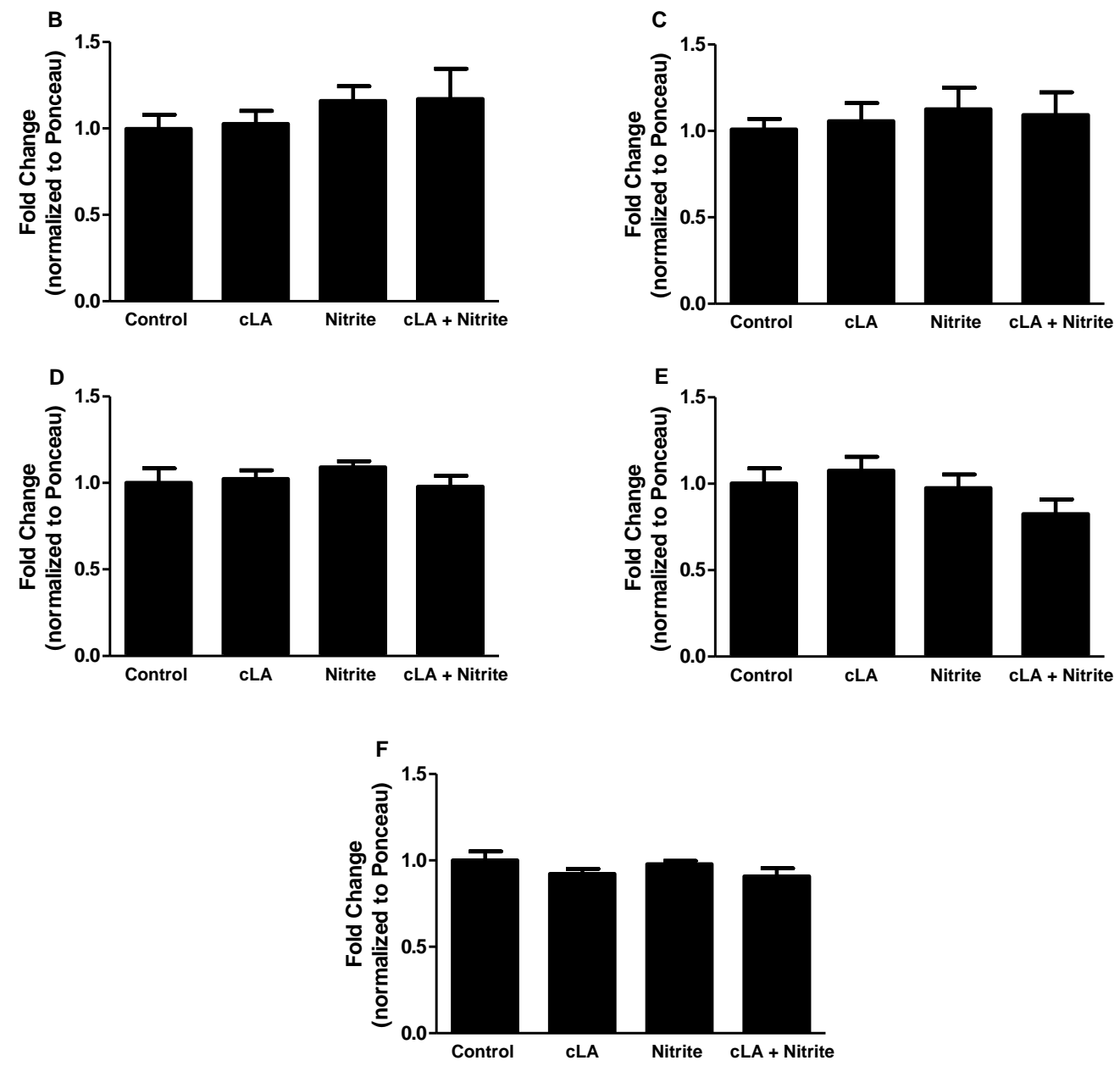
Figure 9. Mitochondrial respiratory chain protein subunit levels remain unchanged following CLA, nitrite or combination treatment with CLA and nitrite. Representative western blot of NDUFB8, SDHB, UQCRC2, MTCO1, and ATP5A (A) protein expression from cardiac tissue of control, CLA, nitrite, and CLA + nitrite treated mice. Quantitated levels of NDUFB8 (B), SDHB (C), UQCRC2 (D), MTCO1 (E) and ATP5A (F) protein expression remain unchanged following $c L A$, nitrite, and $c L A+$ nitrite treatment compared to control ( $n=6$ for all groups). 


\section{Discussion}

The ten day treatment with CLA decreases state 3 respiration (Fig. 7B) and RCR (Fig. 7E) without changes in LEAK (Fig. 7A), state 4 (Fig. 7C) or ETS respiration (Fig. 7D). cLA treatment did not alter mitochondrial respiratory chain subunit proteins (Fig. 9), suggesting the changes in mitochondrial respiration were not due to changes in mitochondrial complex protein levels. CLA has been shown to alter mitochondrial substrate transporter activities $(115,116)$, inhibit beta oxidation (115) and shift skeletal muscle metabolism from oxidative to glycolytic metabolism (133). These results suggest the decrease in state 3 respiration by CLA is mediated by decreases in mitochondrial substrate transporter activities or adenine nucleotide transport.

As nitrite has been associated with cardiac protection (72) and this protection is in part mediated by mitochondria, the impact of nitrite on cardiac mitochondrial function was assessed. Following a 10 day treatment of nitrite, LEAK, state 4, state 3 and ETS respiration (Fig. 7) remained unchanged. Nitrite supplementation has been shown to increase uncoupled mitochondrial respiration (84), and the ETS respiration (Fig. 7D) following nitrite treatment was increased albeit not significantly compared to control. These results show that nitrite does not impact cardiac mitochondrial respiration under baseline conditions. Interestingly, nitrite significantly increased complex I activity (Fig. 8A), while complex II (Fig. 8B), III (Fig. 8C) and IV (Fig. 8D) activity remained

unchanged following nitrite treatment. The change in complex I activity was not 
mediated by increases in complex I protein as NDUFB8, a complex I protein subunit, protein levels did not change (Fig. 9).

Nitrite has been shown to decrease complex I activity $(40,73)$, contrasting the results seen following nitrite treatment (Fig. 8A). The differing results most likely are due to the nitrite treatment on isolated mitochondria (73), ten minute administration of an NO donor (40) and the decrease in complex I activity occurred following cardiac injury. Complex I is prone to S-nitrosylation on multiple subunits (134) which could alter activity suggesting nitrite plays a more dynamic role in regulating complex I activity. Overall, these results demonstrate that nitrite does not alter cardiac mitochondrial respiration under baseline conditions and may play a more prominent role in regulating complex I activity.

As CLA and nitrite are both dietary constituents studying how these dietary components impact cardiac mitochondria together is important. The cis- 9 trans11 isomer of $C L A$ is the preferential substrate for fatty acid nitration in vivo (117) and nitrite can give rise to secondary nitrogen species that mediate nitration of fatty acids.

The co-treatment with $\operatorname{cLA}$ and nitrite was able to decrease state 3 respiration (Fig. 7B) and the RCR (Fig. 7E), without altering LEAK (Fig. 7A), state 4 (Fig. 7C), or ETS (Fig. 7D) respiration. This result mimics the single treatment of CLA on respiratory parameters (Fig. 7), suggesting the decrease in state 3 respiration with the combination treatment is mediated by a cLA-dependent mechanism such as decreased mitochondrial substrate transport (116) or adenine nucleotide transport. 
The co-treatment was able to increase complex I activity (Fig. 8A), while decreasing complex II (Fig. 8B) and III (Fig. 8C) activity. The increased complex I activity (Fig. 8A) and decreased complex II activity (Fig. 8B) seen with the combination treatment of CLA and nitrite can also be linked to a nitrite dependent mechanism for complex I and cLA dependent mechanism for complex II activity. The decrease in complex III activity is dependent on the co-administration of CLA and nitrite and this could rely on the formation of nitrated-cLA. The decreased complex III activity could also potential contribute to the decreased state 3 respiration seen with the combination treatment as complex III is the funnel for all electron flow with the electron transport chain. This would lead to decreased electron flow and state 3 respiration.

Supplementation with CLA and nitrite in healthy humans leads to an increase in nitrated-cLA within the plasma (127) and nitration of CLA occurs within the mitochondria (117). Nitrated fatty acids can post-translationally modify protein thiols and alter activity (135) and the Rieske subunit of complex III contains a thiol that has been shown to be S-nitrosylated within mouse cardiac tissue (134). The unchanged mitochondrial respiratory protein subunits following the co-treatment with CLA and nitrite (Fig. 9) further suggests a post-translational modification mechanism for the reduction in complex III activity.

Overall, these results demonstrate that the dietary constituents' CLA and nitrite can significantly alter cardiac mitochondrial respiratory and electron transport chain activity in vivo. Importantly, CLA and nitrite together can decrease complex III activity, highlighting a complex interaction that occurs following 
consumption of dietary CLA and nitrite that can independently influence cardiac mitochondrial function. 


\title{
CHAPTER IV
}

\section{CO-TREATMENT OF cLA AND NITRITE IN VIVO ATTENUATES CARDIAC MITOCHONDRIAL DYSFUNCTION FOLLOWING MYOCARDIAL INFARCTION Chapter Overview}

\begin{abstract}
Nitrated fatty acids, arising from ischemic cardiac tissue, are cardioprotective in murine models of ischemia reperfusion injury through mitochondrial dependent mechanisms. CLA is the preferred endogenous substrate for fatty acid nitration and previous studies have demonstrated that cotreatment with $\mathrm{CLA}$ and nitrite preserves cardiac function following MI. In this study we examine whether the combination treatment of $C L A$ and nitrite alters mitochondrial function after Ml. We demonstrate that the co-treatment with CLA and nitrite attenuates $\mathrm{Ml}$ induced mitochondrial dysfunction.
\end{abstract}




\section{Introduction}

Myocardial infarction (MI) results from blockage of a coronary artery leading to insufficient blood supply to cardiac tissue, ultimately resulting in cardiomyocyte death (136) and cardiac dysfunction (137). Normal heart function relies on adenosine triphosphate (ATP) generation from mitochondria. Mitochondria produce ATP via the electron transport chain (ETC) to provide energy for the cell. The ETC shuttles electrons from NADH via NADH ubiquinone dehydrogenase (Complex I) or $\mathrm{FADH}_{2}$ via succinate dehydrogenase (Complex II) down the ETC, while pumping protons across the inner mitochondrial membrane creating an electrochemical gradient. ATP synthase (Complex V) uses free energy created by the electrochemical gradient to convert ADP to ATP, while cytochrome c oxidase (Complex IV) transfers electrons from cytochrome $\mathrm{c}$ to oxygen, the final electron acceptor (138). The coupling of electron transport and pumping of protons is essential for ATP synthesis. Mitochondria produce a basal rate of reactive oxygen species (ROS), contributed by complex I, II, and III, and MI results in increased ROS levels (34). The increase in ROS can result in lipid peroxidation (139) and decreased complex I, III and IV activity (34). Decreased ETC enzyme activity leads to decreased mitochondrial respiration following ischemia reperfusion injury (38), indicating that myocardial injury resulting from $\mathrm{Ml}$ is associated with mitochondrial dysfunction.

Numerous studies have highlighted a role for mitochondria in cardioprotection including inhibition of mitochondrial permeability transition pore opening (140, 141), mild mitochondrial uncoupling (142), and mitochondrial 
protein modifications $(40,143)$. Nitrated fatty acids have been shown to mediate cardioprotection in models of ischemia-reperfusion injury, via inhibition of p65 (125) and directly at the mitochondrial level via protein modification of adenine nucleotide translocase-1 (ANT-1) (46).

9Z,11 E-octadecadienoic acid, also known as conjugated linoleic acid or cLA is an 18:2 fatty acid, which due to the nature of the conjugated double bonds, can undergo fatty acid nitration in the mitochondria (117). Recent evidence has shown that a combination treatment of $C L A$ and nitrite is protective following myocardial infarction (88), overall demonstrating that precursors to fatty acid nitration mediate protective cell signaling. Based on these findings, this study investigates how cLA and nitrite impact cardiac mitochondrial function in vivo and following $\mathrm{Ml}$, further evaluating the role of mitochondria in this cardioprotection. 


\section{Results}

To test the hypothesis that co-treatment of CLA and nitrite would mediate cardioprotection via a mitochondrial mechanism; mitochondrial respiration was assessed (as outline in Fig. 5) in cardiac mitochondria from C57BL/6J mice treated with CLA, nitrite and co-treatment prior to and following MI. As treatment was given prior to $\mathrm{MI}$, mitochondrial respiration was assessed following a 3 day treatment prior to $\mathrm{Ml}$ and the combination treatment with $\mathrm{CLA}$ and nitrite had no impact on LEAK (Fig. 10A), state 3 (Fig. 10B) or ETS (Fig. 10C) respiration. The single treatment of CLA decreased LEAK respiration (Fig. 10A) with no change in other mitochondrial respiratory parameters (Fig. 10B-D). The single treatment of nitrite increased both state 3 (Fig. 10B) and ETS (Fig. 10C) respiration and this increase was reflected in the RCR (Fig. 10D).

Electron transport chain activities from complex I and III following 3 day co-treatment revealed that the combination treatment of $\mathrm{CLA}$ and nitrite significantly increased complex III activity (Fig. 11B), while complex I activity (Fig. 11A) remained unchanged. Complex I and III activity were unaltered by the single treatments of CLA and nitrite (Fig. 11A-B). Complex III produces ROS (144), so to determine if the change in complex III activity increased ROS generation, hydrogen peroxide levels were measured in cardiac tissue following 3 day co-treatment. Hydrogen peroxide levels were significantly increased following co-administration of $\mathrm{CLA}$ and nitrite and the single treatment of nitrite

(Fig. 11C). Mitochondrial protein subunit expression was also examined following the 3 day combination treatment and the NDUFB8 protein subunit of complex I 
was significantly decreased following co-treatment (Fig. 12A-B). All other mitochondrial protein subunits were unaffected for CLA, nitrite or the combination of CLA and nitrite (Fig. 12A-F).

Following $\mathrm{Ml}$, state 3 (Fig. 13B) and ETS (Fig. 13C) respiration significantly increased while LEAK respiration (Fig. 13A) and the RCR (Fig. 13D) remained unchanged. Supplementation with CLA was unable to alter MI induced changes in respiration (Fig. 13A-D). However, the single treatment of nitrite was able to attenuate MI-induced increases in state 3 (Fig. 13B) and ETS (Fig. 13C) respiration. Nitrite also increased the RCR (Fig. 13D) following Ml. The combination treatment of cLA and nitrite was able to decrease state 3 (Fig. 13B) and ETS (Fig. 13C) respiration following MI with no change in LEAK respiration (Fig. 13A) or the RCR (Fig. 13D).

Electron transport chain activity was assessed to determine if changes in respiration were linked to alterations in complex I, II, III or IV activity. MI significantly increased complex I (Fig. 14A) and III (Fig. 14C) activity (Fig. 14A), while complex II activity was decreased (Fig. 14B). Complex IV activity (Fig. 14D) was unchanged following MI. CLA treatment further increased complex I activity (Fig. 14A), decreased complex II activity (Fig. 14B), and had no effect on complex III (Fig. 14C) or IV (Fig. 14D) activity following MI. Complex I activity (Fig. 14A) was increased further with nitrite treatment following MI, while complex III (Fig. 14C) and IV (Fig. 14D) activity remained unaltered with nitrite following Ml; however, nitrite was able to restore complex II activity (Fig. 14B) to control levels following MI. 
The co-treatment with CLA and nitrite increases complex I activity following MI (Fig. 14A), had no effect on complex II activity (Fig. 14B), and was able to attenuate the MI-induced increase in complex III activity (Fig. 14C). Mitochondrial respiratory protein subunit expression revealed $\mathrm{MI}$ increased all respiratory subunit expression levels (Fig. 15A-F) and CLA, nitrite or co-treatment had no effect (Fig. 15). MI can cause increases in ROS production (34) and mitochondrial antioxidants play an important role in balancing ROS production. MnSOD protein expression was examined following $\mathrm{MI}$ and the co-treatment of CLA and nitrite significantly increased MnSOD protein expression after MI (Fig. 16). Due to the combination treatment decreasing complex III activity and increasing MnSOD, hydrogen peroxide levels were assessed to determine if ROS production was changed following Ml. Hydrogen peroxide levels are increased following $\mathrm{MI}$ (Fig. 17) and the single treatment of nitrite and cotreatment with CLA and nitrite is able to attenuate the increased hydrogen peroxide following MI (Fig. 17). 
Figure 10. Mitochondrial respiration remains unchanged following co-treatment with cLA and nitrite.

A

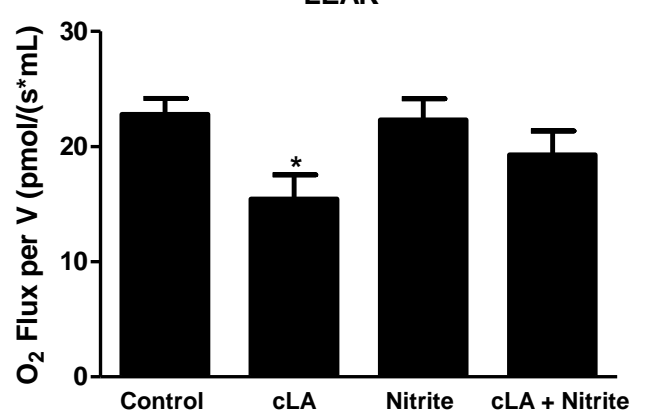

C

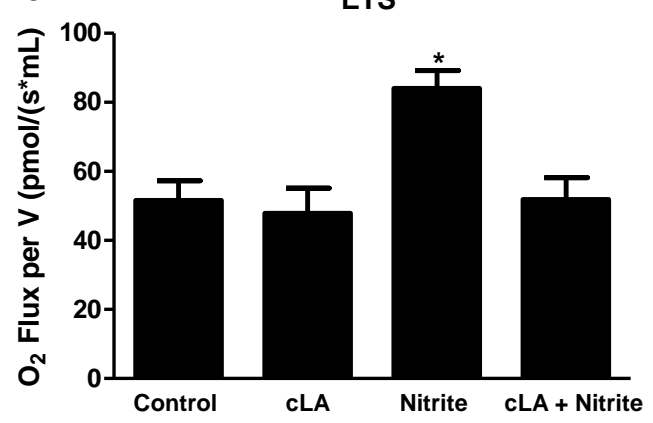

B

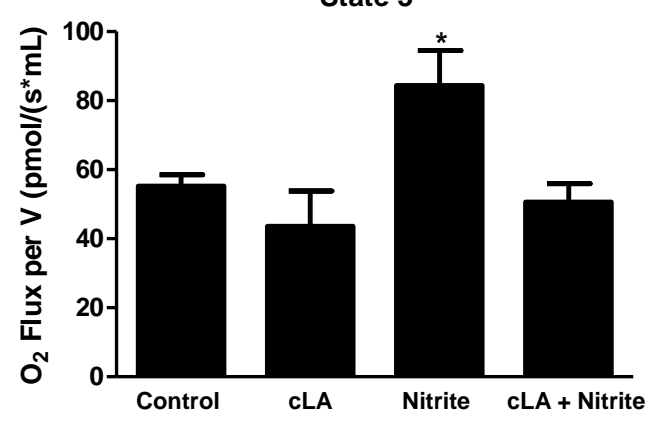

D

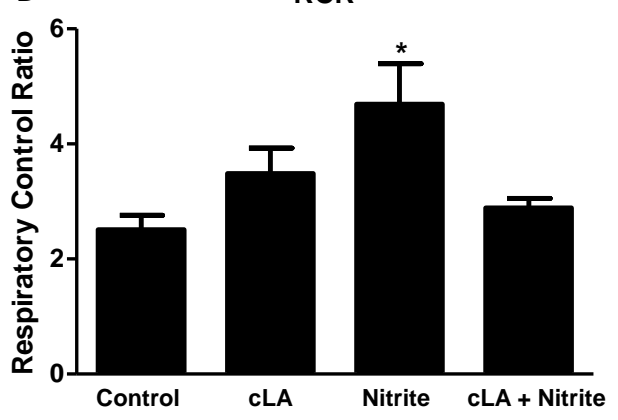

Figure 10. Mitochondrial respiraiton remains unchanged following cotreament with cLA and nitrite. Three day $\mathrm{CLA}+$ nitrite treatment has no effect on LEAK ( $A$, control $n=8, \operatorname{cLA} n=9$, nitrite $n=8, c L A+$ nitrite $n=12)$, state 3 ( $B$, control $n=8, c L A n=7$, nitrite $n=7, c L A+$ nitrite $n=11)$ or $E T S(C$, control $n=8, c L A$ $n=9$, nitrite $n=8, c L A+$ nitrite $n=11$ ) respiration. $\operatorname{cLA}$ decreases LEAK (A) respiration, while nitrite increases state $3(B)$ respiration, ETS $(C)$ respiration and the RCR (D, control $n=8$, cLA $n=9$, nitrite $n=6$, cLA + nitrite $n=11) .\left({ }^{*} p<0.05\right.$ compared to control, One way ANOVA). 
Figure 11. Co-treatment with cLA and nitrite increases complex III activity and nitrite increases $\mathrm{H}_{2} \mathrm{O}_{2}$ levels.
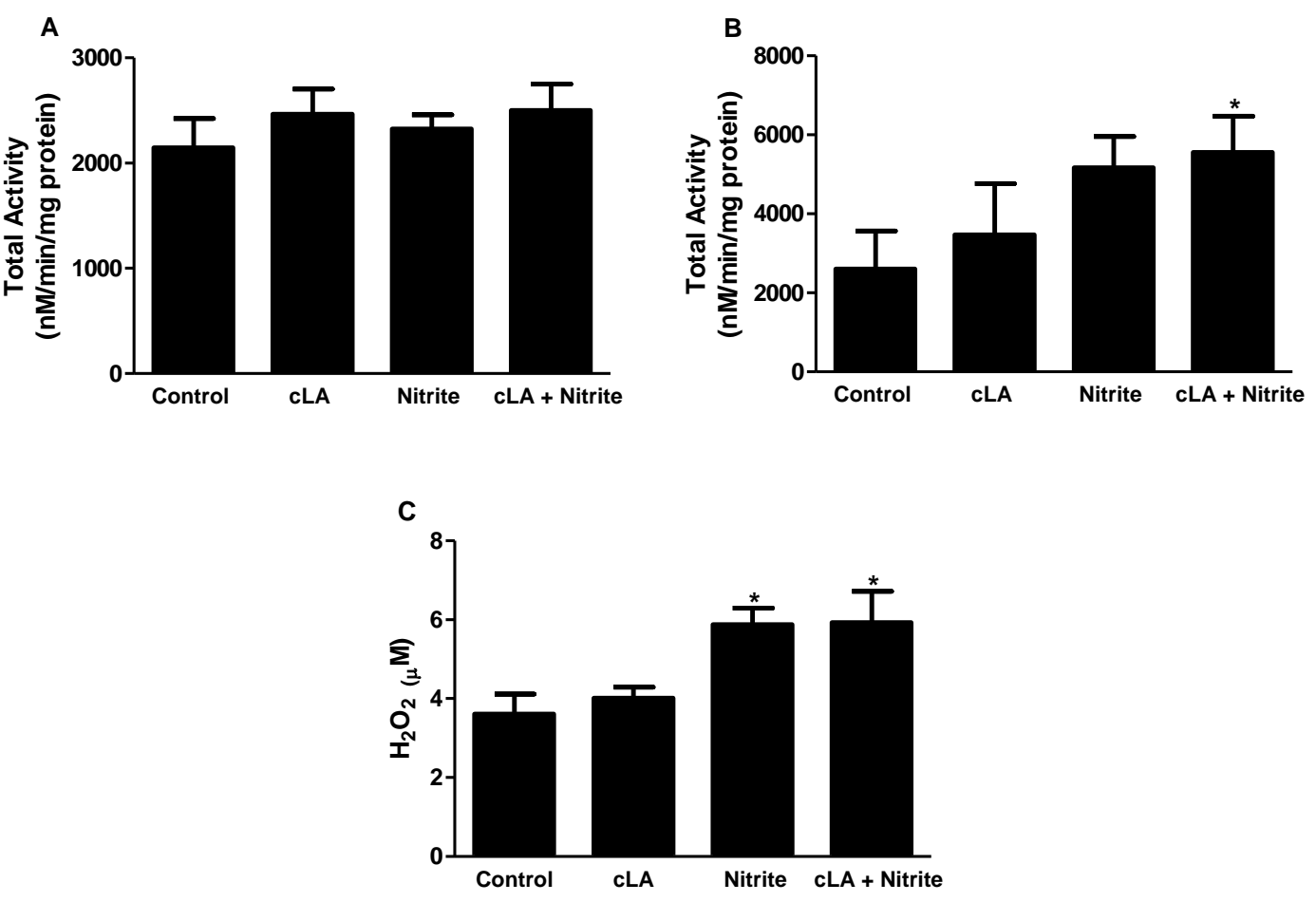

Figure 11. Co-treatment with cLA and nitrite increases complex III activity and nitrite increases $\mathrm{H}_{2} \mathrm{O}_{2}$ levels. Complex III activity (B) significantly increases following three day combination treatment and $\mathrm{H}_{2} \mathrm{O}_{2}$ levels significantly increases following nitrite treatment $(C)$ while complex I actvitity $(A)$ remains unchanged. ( ${ }^{*} \mathrm{p}<0.05$ compared to control, unpaired student $\mathrm{t}$-test, $\mathrm{n}=4$ for all groups). 
Figure 12. Co-treatment with cLA and nitrite decreases NDUFB8 protein expression.
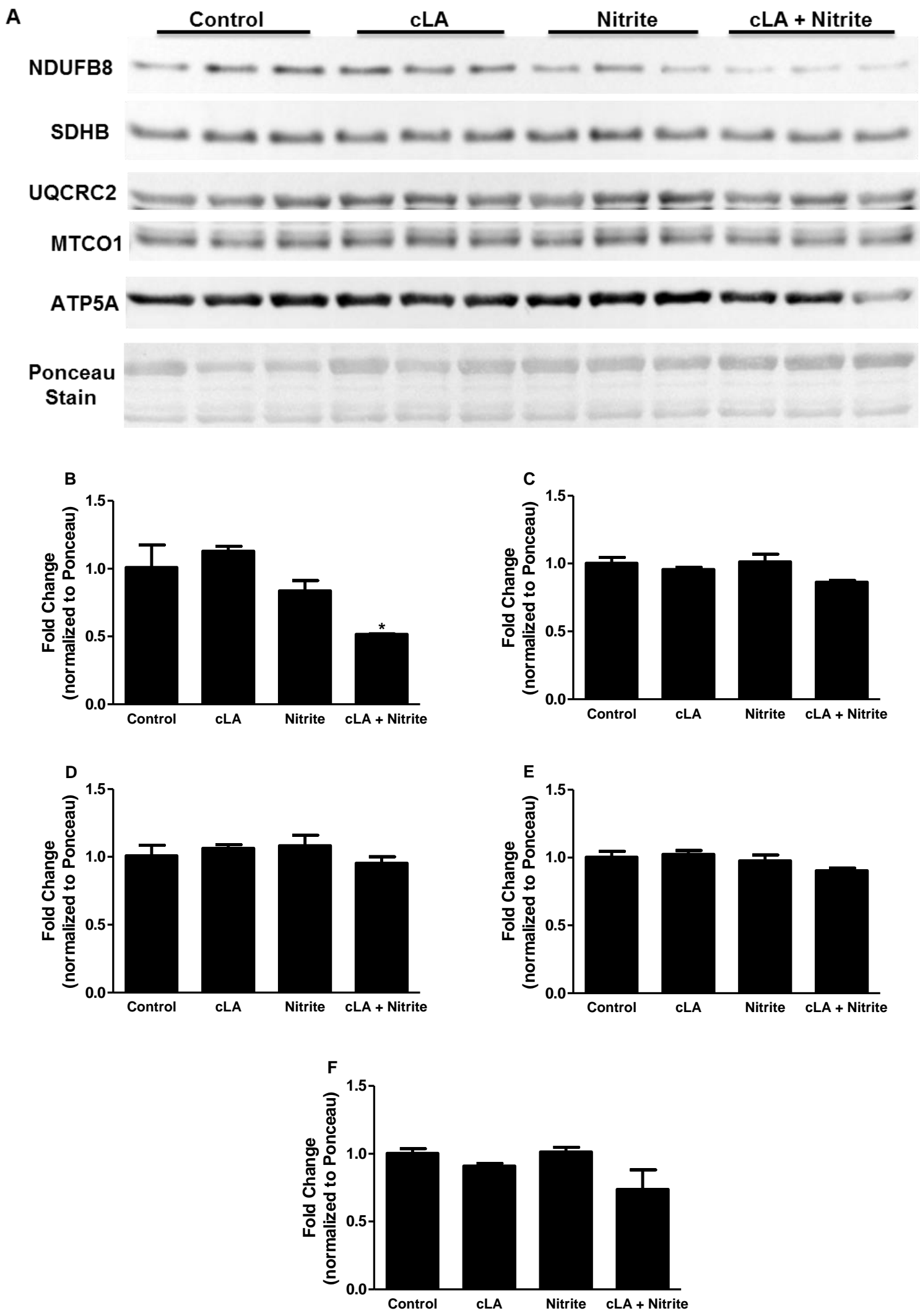
Figure 12. Co-treatment with CLA and nitrite decreases NDUFB8 protein expression. NDUFB8 protein expression (A-B) is significantly decreased following $\mathrm{CLA}+$ nitrite co-treatment. SDHB ( $A$ and $C$ ), UQCRC2 (A and D), MTCO1 (A and E), and ATP5A (A and F) protein levels remain unchanged following $C L A$, nitrite and co-treatment. $\left({ }^{*} p<0.05\right.$ vs control, One way ANOVA, $\mathrm{n}=3$ for all groups). 
Figure 13. Nitrite attenuates increases in State 3 and ETS respiration following MI.
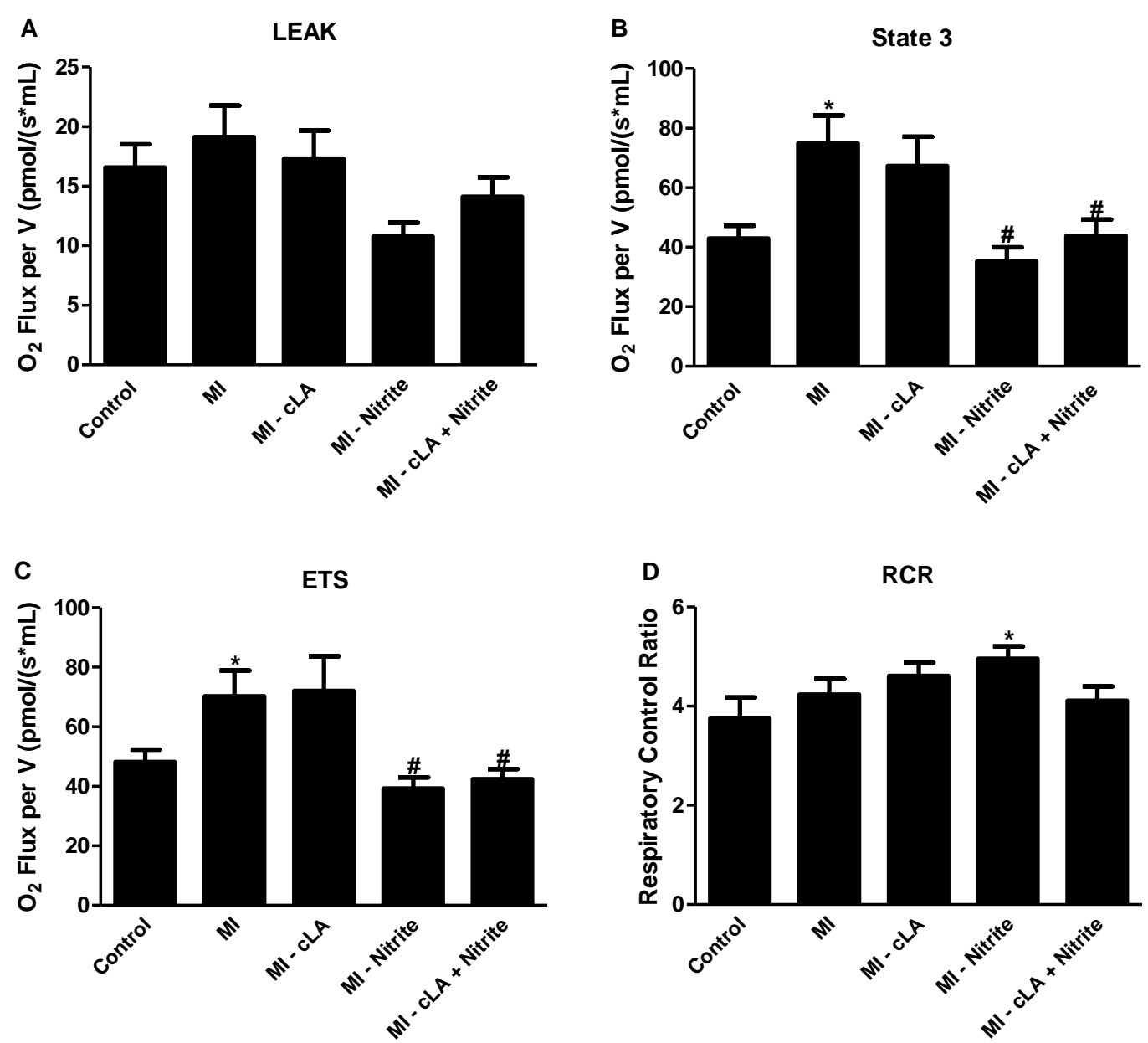

Figure 13. Nitrite attenuates increases in State 3 and ETS respiration following MI. Ml increases state $3(B$, control $n=8, M I n=6, M I-c L A ~ n=7, M I-$ Nitrite $n=5, M I-\operatorname{cLA}+$ nitrite $n=8)$ and $E T S(C$, control $n=8, M I n=6, M I-\operatorname{cLA} n=7$, MI - Nitrite $n=5, M I$ - $c L A+$ nitrite $n=8$ ) respiration while LEAK respiration (A, control $n=8, M I n=5, M I-c L A n=7, M I-$ Nitrite $n=5, M I-c L A+$ nitrite $n=8$ ) and the RCR (D, control $n=6, M I n=6, M I-c L A n=7, M I$ - Nitrite $n=5, M I-c L A+$ nitrite $\mathrm{n}=8$ ) remain unchanged. Supplementation with nitrite and $c L A+$ nitrite decreases State $3(B)$ and ETS $(C)$ respiration following $\mathrm{Ml}\left({ }^{*} \mathrm{p}<0.05\right.$ compared to control, $\# p<0.05$ compared to $\mathrm{Ml}$, unpaired student $\mathrm{t}$-test). 
Figure 14. Co-treatment with cLA and nitrite attenuates increased complex III activity following MI.
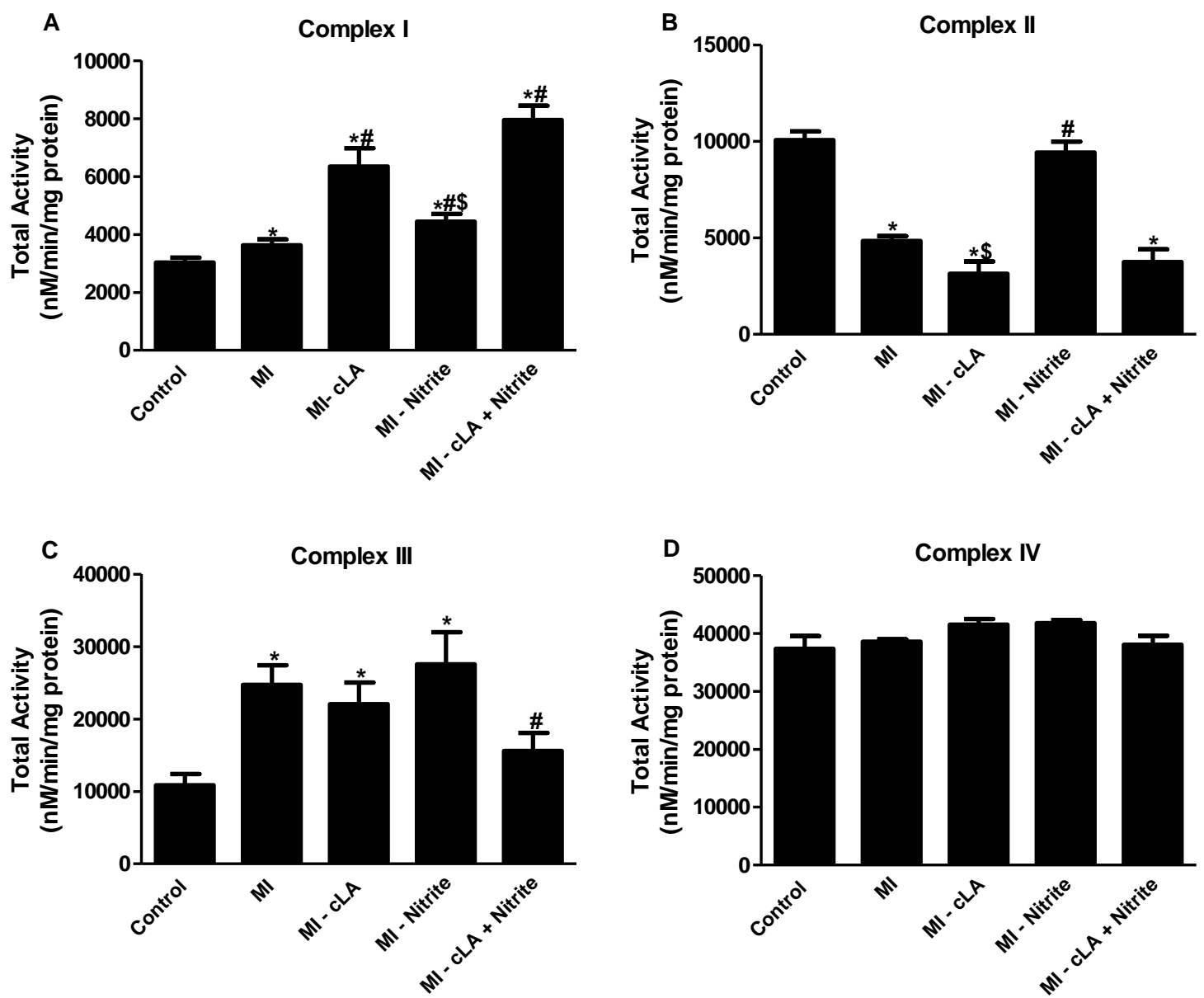

Figure 14. Co-treatment with cLA and nitrite attenuates increased complex III activity following MI. MI increases complex I (A) and complex III (C) activity, decreases complex II (C) activity and has no effect on complex IV activity (D). CLA increases complex I (A) activity, decreases II (B) activity and has no effect on complex III (C) or IV (D) activity following MI. Nitrite increases complex I (A) activity, restores complex II (B) activity, and has no effect on complex III (C) or IV (D) activity following MI. Co-treatment with $\mathrm{CLA}+$ nitrite increases complex I (A) activity, decreases complex III (C) activity and has no effect on complex II (B) or 


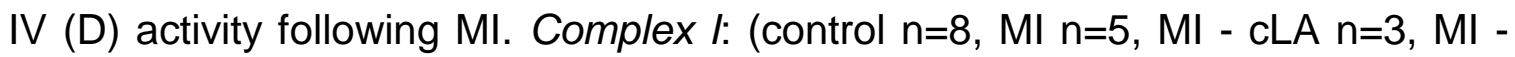
Nitrite $n=4, M I-c L A+$ nitrite $n=6,{ }^{*} p<0.05$ compared to control, $\# p<0.05$ compared to $\mathrm{Ml}, \$ \mathrm{p}<0.05$ compared to $\mathrm{Ml}$ - cLA, $\mathrm{MI}$ - $\mathrm{cLA}+$ nitrite, One way

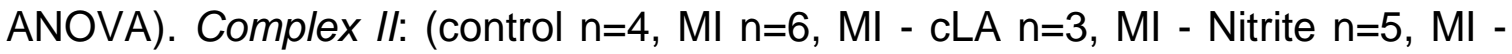
$c L A+$ nitrite $n=6,{ }^{*} p<0.05$ compared to control, $\# p<0.05$ compared to MI, MIcLA, and MI- cLA + nitrite, $\$ p<0.05$ compared to MI, One way ANOVA). Complex

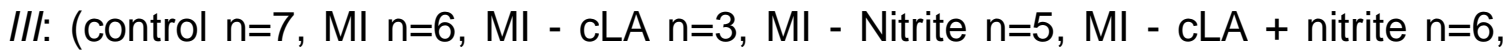
${ }^{*} \mathrm{p}<0.05$ compared to control, $\# \mathrm{p}<0.05$ compared to $\mathrm{Ml}$ and Ml- Nitrite, One way ANOVA). Complex IV: (control $\mathrm{n}=4, \mathrm{MI} n=6, \mathrm{MI}-\mathrm{cLA} n=3, \mathrm{MI}$ - Nitrite $\mathrm{n}=5$, MI $c L A+$ nitrite $n=6)$. 
Figure 15. Treatment with cLA, nitrite or combination increases mitochondrial respiratory chain protein expression following MI.

A
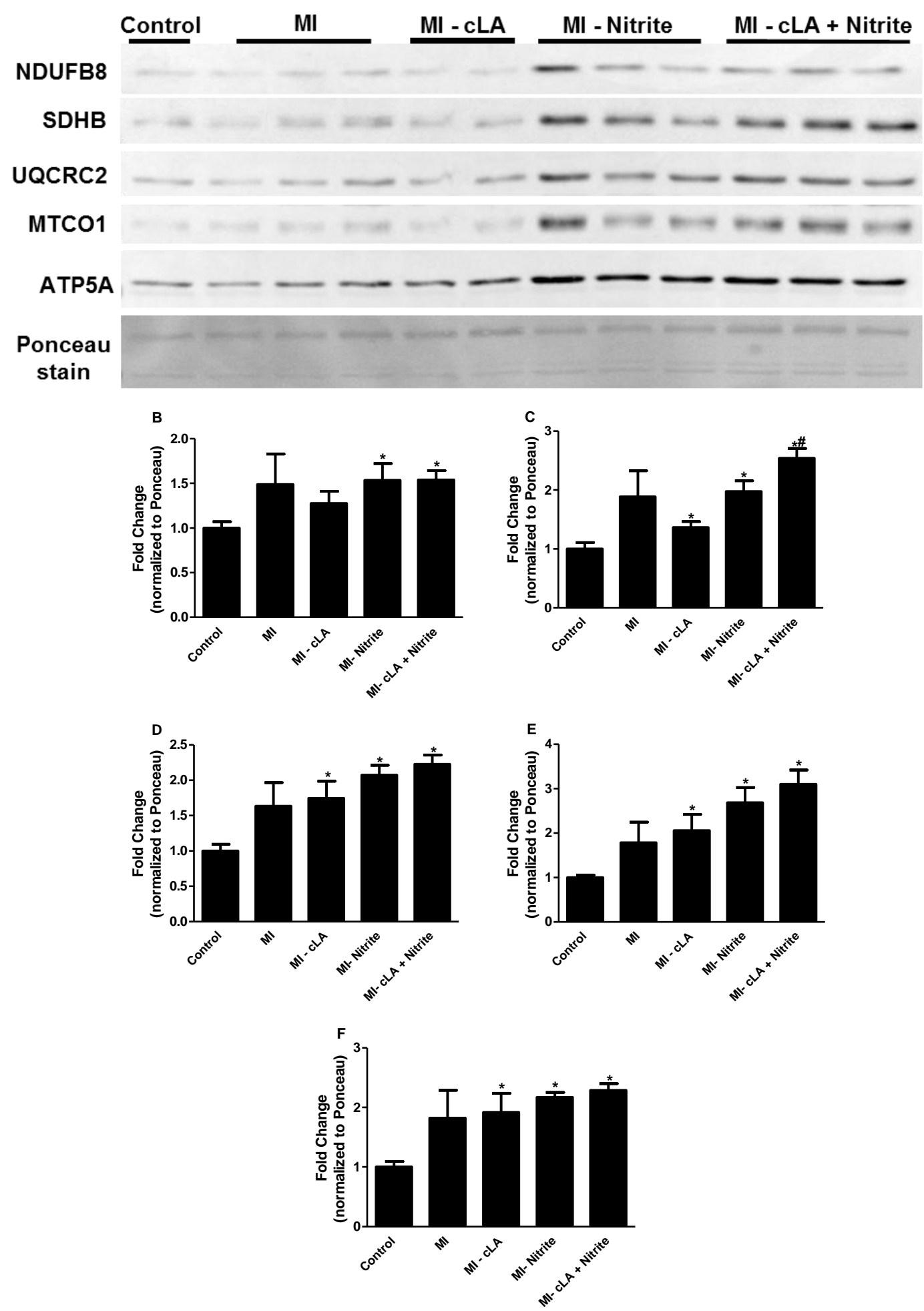
Figure 15. Treatment with cLA, nitrite or combination increase mitochondrial respiratory protein expression following MI. Representative western blot of NDUFB8, SDHB, UQCRC2, MTCO1, and ATP5A (A) protein expression from isolated cardiac mitochondria from control, MI, MI - CLA, MI Nitrite and MI - CLA + nitrite. MI- CLA increases SDHB (C), UQCRC2(D), MTCO1 (E) and ATP5A (F) protein expression ( ${ }^{*} \mathrm{p}<0.05$ compared to control). $\mathrm{MI}$ - Nitrite and $\mathrm{MI}$ - CLA + nitrite increases NDUFB8 (B), SDHB (C), UQCRC2 (D), MTCO1 (E) and ATP5A $(F)$ protein expression $\left({ }^{*} p<0.05\right.$ compared to control, $\# p<0.05$ compared to MI - cLA, MI - Nitrite, unpaired student t-test). 
Figure 16. Treatment with cLA + nitrite increases MnSOD protein expression following MI.
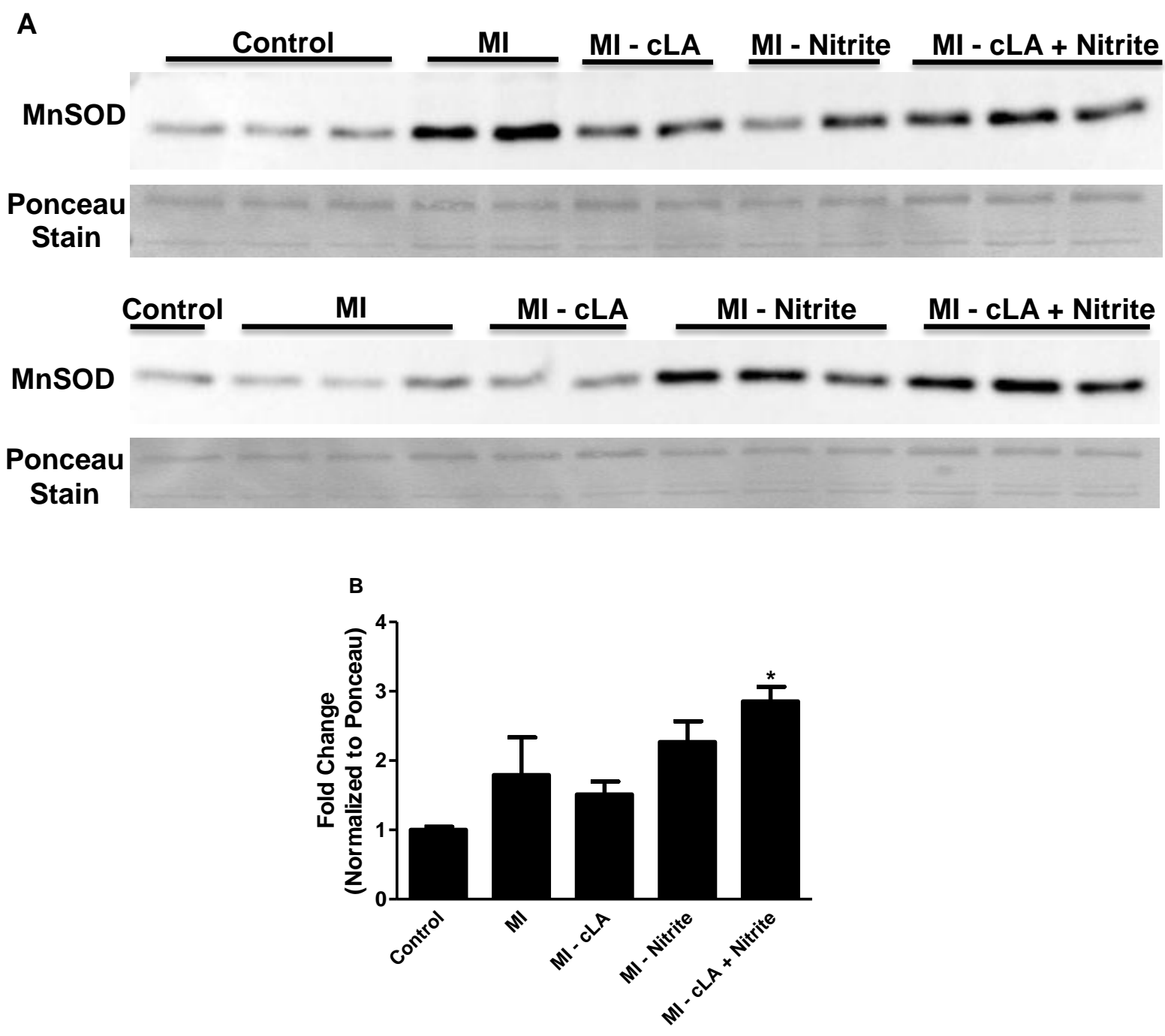

Figure 16. Treatment with CLA + nitrite increases MnSOD protein expression following MI. Representative western blots of MnSOD (A) protein expression from isolated cardiac mitochondria from control $(n=4), M I(n=5), M I-$ $\operatorname{cLA}(n=4), M I$ - Nitrite $(n=5)$ and MI - cLA + nitrite $(n=6)$. Co-treatment with cLA and nitrite increases MnSOD protein expression following $\mathrm{MI}(\mathrm{B}) \quad\left({ }^{*} \mathrm{p}<0.05\right.$ compared to control, unpaired student t-test). 
Figure 17. Nitrite decreases $\mathrm{H}_{2} \mathrm{O}_{2}$ levels following MI.

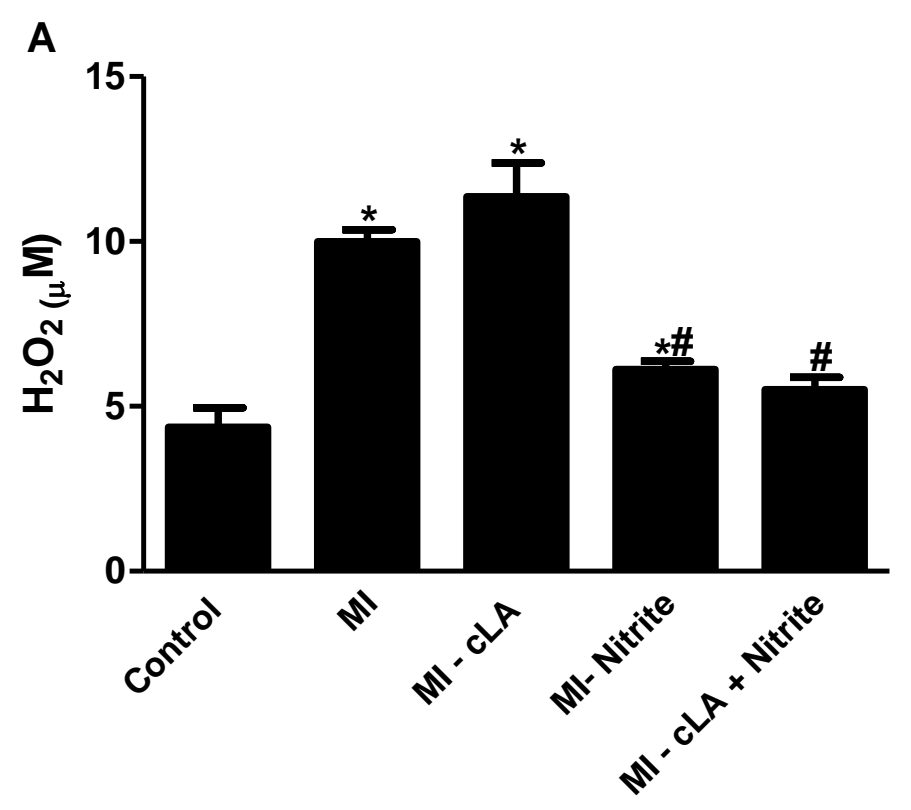

Figure 17. Nitrite decreases $\mathrm{H}_{2} \mathrm{O}_{2}$ levels following $\mathrm{MI} . \mathrm{H}_{2} \mathrm{O}_{2}$ levels increase following $\mathrm{MI}(n=4), M I-\operatorname{cLA}(n=3)$ and MI - Nitrite $(n=4)\left({ }^{*} p<0.05\right.$ compared to control, $n=4$, One way ANOVA). However, supplementation with nitrite or $\mathrm{CLA}+$ nitrite $(n=7)$ decreases $\mathrm{H}_{2} \mathrm{O}_{2}$ production following $\mathrm{Ml}$ (\#p<0.05 compared to $\mathrm{Ml}$ and MI- CLA, One way ANOVA). 


\section{Discussion}

Recent evidence has shown that co-treatment with CLA and nitrite mediates protection against MI (88). CLA undergoes nitration in vivo (117) and nitrated fatty acids mediate protection against ischemic injury by mitochondrial dependent mechanisms $(47,126)$. Cardiac mitochondrial respiration and respiratory complex activity was assessed prior to and following $\mathrm{Ml}$ with cotreatment of $\mathrm{CLA}$ and nitrite to determine whether mitochondria played a role in this protection. The major findings from this study reveal that co-treatment with CLA and nitrite is able to attenuate $\mathrm{Ml}$-induced increases in state 3 respiraiton (Fig. 13 B), ETS respiration (Fig. 13C), complex III activity (Fig. 14C) and hydrogen peroxide levels (Fig. 17), while increasing MnSOD protein expression following MI (Fig. 16).

The combination treatment was administered 3 days prior to $\mathrm{Ml}$ and mitochondrial respiration was performed following 3 day treatment to determine mitochondrial changes ocurring prior to MI. The combination treatment of $\mathrm{CLA}$ and nitrite did not alter mitochondrial respiration parameters including: LEAK respiration (Fig. 10A), state 3 respiration (Fig. 10B), ETS respiration (Fig. 10C) and the RCR (Fig. 10D). These results indicate that mitochondrial respiration is functional with the co-treatment prior to MI.

The co-treatment prior to MI was able to significantly increase complex III activity (Fig. 11B) without changing complex I activity (Fig. 11A). The combination treatment did decrease NDUFB8 protein expression (Fig. 12A and B) without changing SDHB, UQCRC2, MTCO1 and ATP5A protein levels (Fig. 12 
A-F). The NDUFB8 is not involved in the catalytic activity of complex I, thereby potentially explaining the unaltered complex I activity seen with the combination treatment despite decreased NDUFB8 protein levels.

As complex I and III can produce ROS (38), hydrogen peroxide levels were measured to determine if the increase in complex III activity correlated with an increase in ROS. Hydrogen peroxide levels were increased with both nitrite and the combination of CLA and nitrite (Fig. 11) indicating nitrite is increasing hydrogen peroxide levels independent of changes in complex III activity. However, the hydrogen peroxide was measured in cardiac tissue homogenate thereby the source of hydrogen peroxide could differ between the nitrite treatment and co-treatment as nitrite can increase NADH oxidase 2 (NOX2), a mediator of non-mitochondrial ROS generation (107).

Following MI, state 3 respiration (Fig. 13B) and ETS respiration (Fig. 13C) increased without changing LEAK respiration (Fig. 13A) or the RCR (Fig. 13D). The increases in respiration following $\mathrm{Ml}$ are in contrast to reports in the literature in which $\mathrm{Ml}$ is associated with mitochondrial dysfunction (34). The differences observed in this study may be related to the time point, as mitochondrial respiration was assessed ten days after $\mathrm{Ml}$ and most studies evaluate mitochondrial function within a short time period. Additionally, this time period allows for adaptive cell signalling to compensate for mitochondrial dysfunction and the increased mitochondrial respiratory protein levels following MI (Fig. 15AF) indicates an upregulation in electron transport chain protein expression to compensate for mitochondrial dysfunction. 
The single treatment of $\mathrm{CLA}$ was unable to alter changes in mitochondrial respiration induced by Ml. However, single treatment of nitrite and combination treatment of $C L A$ and nitrite was able to attenuate increased state 3 respiration (Fig. 13B) and ETS respiration (Fig. 13C) following Ml. The reliance on nitrite to reduce mitochondrial respiration following $\mathrm{Ml}$ in the single and combination treatment could be attributed to nitrite's conversion to nitric oxide. Nitrite can undergo reduction to nitric oxide under conditions of hypoxia by deoxygenated hemoglobin (62), deoxygenated myoglobin (63), and low pH (145), all conditions that prevail following MI. Nitric oxide can inhibit mitochondrial respiration (81) demonstrating that the nitrite mediated decreases in respiration observed following MI could be linked to nitrite's conversion to nitric oxide.

MI increased complex I activity (Fig. 14A) and CLA, nitrite and the combination treatment further increased complex I activity (Fig. 14A). The increased complex I activity following $\mathrm{MI}$ is contrary to literature in which cardiac injury decreases complex I activity (34). The observed differences in this study could be due to the time point in which complex I activity was assessed (10 days) or the area of cardiac tissue from which mitochondria were isolated (border zone vs whole heart). Both the $C L A$ and co-treatment of $C L A$ and nitrite are significantly higher than the $\mathrm{MI}$ and $\mathrm{MI}$ - Nitrite group indicating $\mathrm{CLA}$ is the mediator of this increase. CLA has been shown to increase ROS in macrophages (105), oxidative stress in rat cardiac tissue (106), and 8-iso-prostaglandin F2a urine levels in obese males (104). cLA has also been shown to induce cardiac dysfunction in aging by uncoupled eNOS (107). These results suggest that CLA may exacerbate 
oxidative stress conditions such as $\mathrm{Ml}$, aging and obesity. The increased complex I activity observed with cLA following MI may provide a mechanistic link between CLA and the detrimental effects observed with CLA in oxidative stress conditions.

Complex II activity was significantly decreased following MI (Fig. 14B) and neither CLA nor the co-treatment was able to restore complex II activity after MI (Fig. 14B). Nitrite administration restored complex II activity following MI (Fig. 14B); however, the restoration of complex II activity is not necessary to mediate cardioprotection as nitrite was unable to provide protection against MI (88).

MI significantly increased complex III activity (Fig. 14C) and the single treatments of CLA and nitrite were unable to decrease $\mathrm{Ml}$ induced upregulation of complex III activity. The increased complex III activity following MI differs from literature reports in which cardiac injury decreases complex III activity (34) and this is most likely due to the border zone of the infarct being used for cardiac mitochondrial complex III activity as opposed to whole heart.

The combination of $\operatorname{cLA}$ and nitrite was able to attenuate the increased complex III activity following MI (Fig. 14C). The decreased complex III activity mediated by $C L A$ and nitrite could rely on the formation of nitrated $C L A$ since $C L A$ undergoes nitration within mitochondria (117) and formation of nitrated fatty acids increases following ischemic injury (125). The Reiske subunit of complex III contains a thiol that can be S-nitrosylated (134) and the alkanine conditions within mitochondria promote nitro-fatty acids to react with thiols (135). These 
conditions favor a mechanism by which nitrated $C L A$ is able to post-translationally modify complex III, thereby decreasing its activity following MI.

High levels of ROS generated by complex I and III during MI can result in cardiac damage and upregulated complex I and III activity following MI correlated with increases in hydrogen peroxide after MI (Fig. 17). Supplementation with nitrite and the combination of cLA and nitrite was able to attenuate the increased hydrogen peroxide levels (Fig. 17), while the single treatment of CLA was unable to decrease the levels (Fig. 17). The decreased complex III activity following cotreatment of CLA and nitrite could explain the decreased hydrogen peroxide levels generated after $\mathrm{Ml}$; however the single treatment of nitrite also decreased the hydrogen peroxide levels. An alternative explanation could be attributed to nitrite derived NO reaction with superoxide thereby reducing the pool of superoxide that can be converted to hydrogen peroxide.

Overall, these results suggest the cardiac protection afforded by the combination treatment of cLA and nitrite may be in part mediated by attenuation of $\mathrm{MI}$ induced increases in mitochondrial respiration, complex III activity and ROS generation. 


\section{CHAPTER V}

\section{CARDIAC MITOCHONDRIAL FUNCTION AND AGING}

\section{Chapter Overview}

Aging and ischemic injury share similarities of cardiac mitochondrial dysfunction resulting in decreased heart function. CLA and nitrite are found within dietary foods such as runimant meat and green leafy vegetables. Nitrite and cLA have both been associated with cardiovascular health and appear to mediate these effects through mitochondria. However, the impact of nitrite and CLA on cardiac mitochondria in aging is unknown. Recent evidence has shown that $\mathrm{CLA}$ supplementation in aged mice decreased cardiac function and supplementation of nitrite was able rescue this dysfunction. This study examines the impact of aging on cardiac mitochondrial function and examines whether co-treatment with cLA and nitrite rescues cardiac mitochondrial dysfunction. This study reveals there is an age dependent decline in cardiac mitochondrial function and supplementation with the combination treatment of $C L A$ and nitrite was unable to rescue aged related cardiac mitochondrial function. 


\section{Introduction}

Aging is associated with cellular changes ultimately resulting in declines in cellular function (146). The development of cardiovascular disease and risk for Ml increases with age (146). Aging is associated with increased oxidative stress (147) and mitochondrial driven ROS contributes to this elevated oxidative stress condition. Aging results in diminshed mitochondrial function (148-150) thereby limiting the ATP pool needed to maintain normal cardiovascular function. Mitochondria also play an important role in mediating cardioprotective effects against MI (32); however, these protective effects are lost in aging (42). One of the theories behind this loss of cardioprotection is loss of mitochondrial function (42), so strategies to improve mitochondrial function in aging could restore mitochondrial mediated protection against MI. Nitrite and CLA are dietary consitutents that are known to influence mitochondrial function $(73,115)$ and mediate cardioprotection (88). However, it is unknown if these effects carry over to aged mitochondria. This study was undertaken to determine whether cLA, nitrite or the combination is able to reverse aged cardiac mitochondrial dysfunction. 


\section{Results}

Mitochondrial respiration assessed in 10 and 33 month old revealed an aged dependent decline in mitochondrial respiratory capacity. Ten-month old mice displayed a significant decrease in state 3 respiration (Fig. 18B) with a concurrent decrease in the RCR (Fig. 18D). LEAK respiration (Fig. 18A) was not decreased in 10 month old mice. The ETS respiration (Fig. 18C) appeared to be decreased, though it was not statistically significant. When mice were aged to 33 months, there was a significant decline in both LEAK (Fig. 18A) and state 3 (Fig. 18B) respiration compared to 8-12 week old mice. The ETS respiration was noticeable decreased in 33 month old mice (Fig. 18C), but was not statistically significant. The RCR (Fig. 18D) was not changed. To assess whether supplementation with CLA and nitrite could rescue aged related declines in mitochondrial function, 10 month old mice were treated with $\mathrm{CLA}$, nitrite or the combination treatment of CLA and nitrite for 6 weeks after which mitochondrial function was assessed. Treatment with CLA, nitrite or co-treatment did not alter LEAK respiration (Fig. 19A) or rescue decreased state 3 respiration (Fig. 19B). 
Figure 18. Aging induces cardiac mitochondrial dysfunction.

A

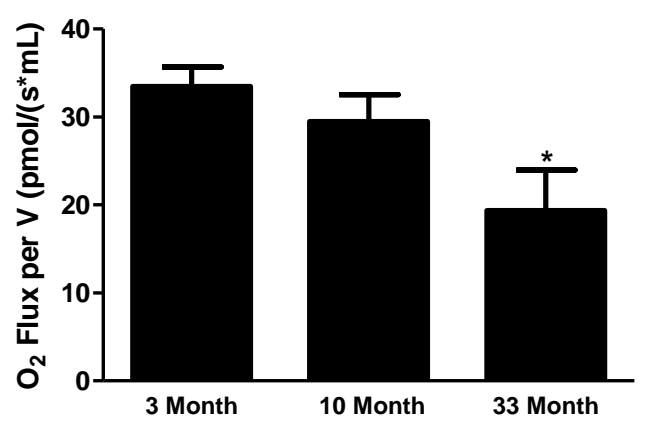

C

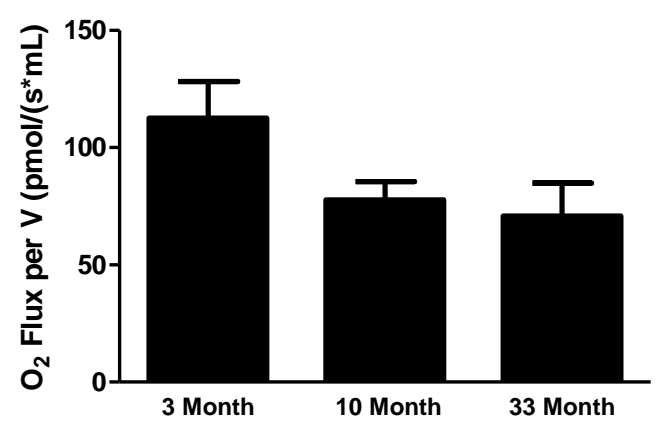

B

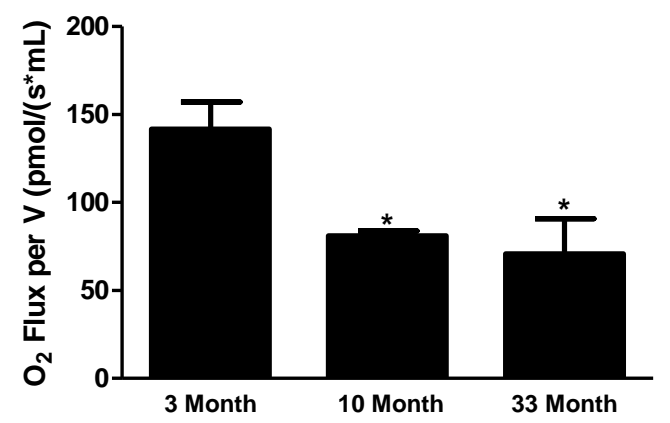

D

RCR

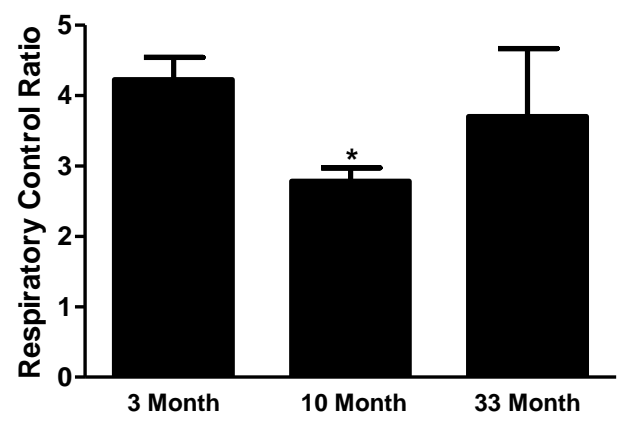

Figure 18. Aging induces cardiac mitochondrial dysfunction. 10 month old mice have reduced state $3(B)$ respiration, ETS respiration $(C)$ and $R C R(D)$, while LEAK respiration $(A)$ remained unchanged. LEAK $(A)$, state $3(B)$ and ETS (C) respiration was decreased in 33 month old mice, while the $R C R$ (D) remained unchanged. ( ${ }^{*} p<0.05$ vs 3 month, unpaired student t-test, 3 month $n=4,10$ and 33 month $n=3)$. 
Figure 19. Treatment with cLA, nitrite, or in combination does not attenuate aged related decline in cardiac mitochondrial function.
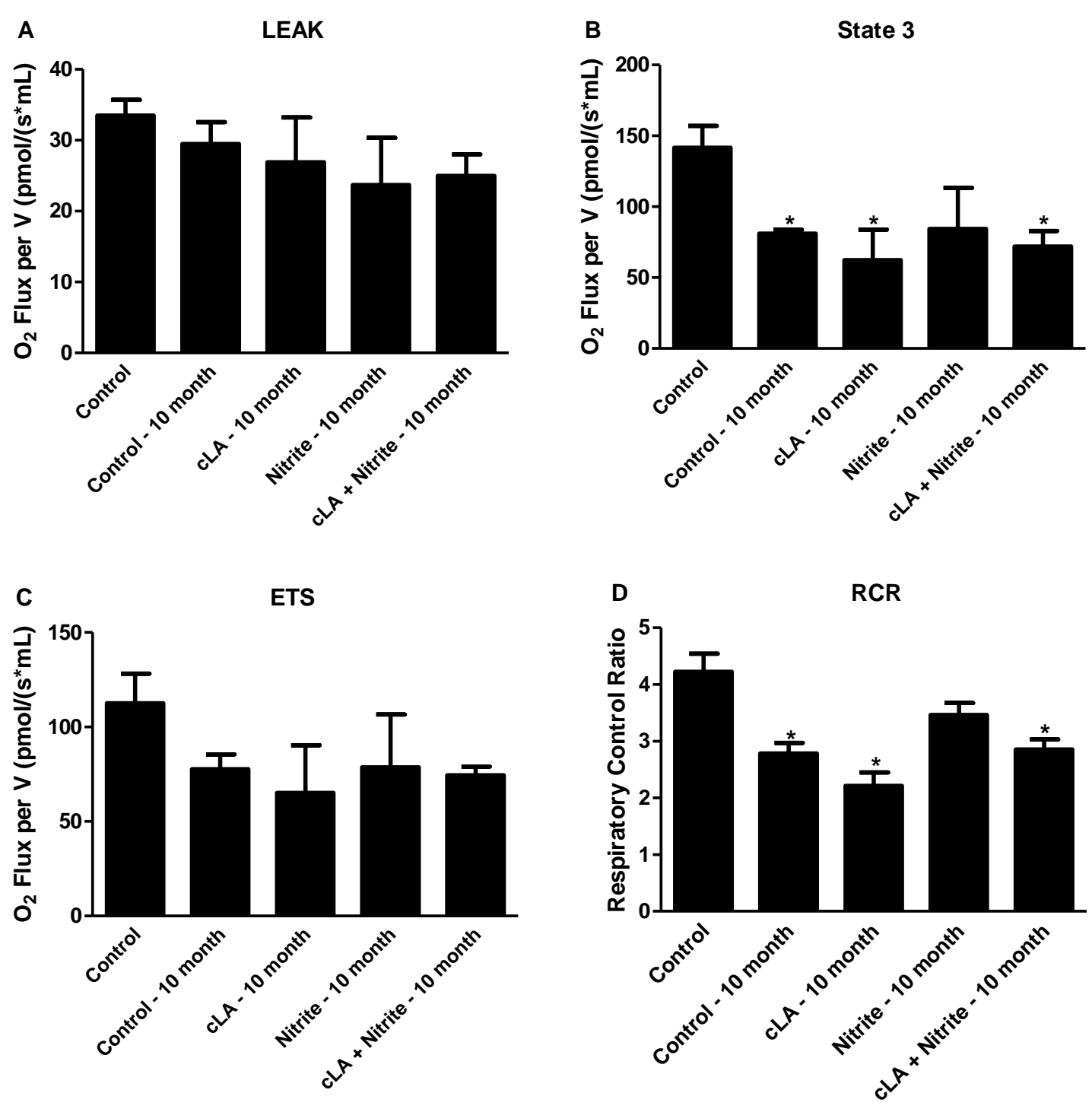

Figure 19. Treatment with CLA, nitrite or in combination does not attenuate aged related decline in cardiac mitochondrial function. Six week treatment with $\operatorname{CLA}$, nitrite and $\operatorname{CLA}+$ nitrite was unable to rescue reduced state 3 respiration (B), ETS respiration (C) or the RCR (D). LEAK (A) respiration was unchanged following treatment with $c L A$, nitrite or $c L A+$ nitrite. $\left({ }^{*} p<0.05\right.$ vs 
control, One way ANOVA, control $n=4$, control - 10 month, $c L A-10$ month, Nitrite

- 10 month, and cLA + nitrite -10 month $n=3$ ). 


\section{Discussion}

Age is a significant risk factor for cardiovascular disease and subsequent complications such as MI (151). The development of cardiovasuclar disease in aging is associated with mitochondrial dysfunction and increased oxidative stress (147). Mitochondrial generated ATP is essential for maintaining normal cardiovascular health and plays a protective role against MI (32). However, with aging, the protective role of mitochondria against $\mathrm{Ml}$ is lost (42) and this is thought to be associated with a decline in mitochondrial function. As demonstrated in this study, mice aged to 10 or 33 months displayed decreased mitochondrial function (Fig. 18). The 33 month-old mice had significantly decreased LEAK (Fig. 18A) and state 3 (Fig. 18B) respiration, while the 10 month-old mice only had a decrease in state 3 respiration (Fig. 18B).

The decrease in state 3 respiration but not LEAK respiration for the 10 month-old mice suggests that under low energy demand the ETS is able to effectively transport electrons down the ETS. However, under high energy demands requiring production of ATP, the ETS is unable to meet these demands, resulting in supressed state 3 respiration. As aging continues, the ETS further declines in electron transporting capacity, resulting in the diminished LEAK and state 3 respiration observed in the 33 month-old mice. This can result in over production of mitochondrial generated ROS (152) coupled with reduced antioxidant defenses (153), ultimately resulting in damage to the oxidative phosphorylation proteins within the ETS and leading to mitochondrial dysfunction. The ETS was reduced in both 10 and 33 month old-mice; however, 
it was not statistically significant. This is likely due to the low number of mice per group and over titration of the mitochondrial uncoupler resulting in greater variability within the ETS respiration. Overall, these data shows there is an age dependent decline in mitochondrial function.

Nitrite is considered an endogenous reservoir of NO that can be obtained from the diet by consuming green leafy vegetables or beet juice. Nitrite has been shown to increase mitochondrial function in adipocytes (83) and modulate cardiac mitochondrial function under injury settings (51); however the effect of nitrite on aged cardiac mitochondrial function is unknown. This study assessed whether nitrite supplementation could rescue aged related cardiac mitochondrial dysfunction and found that it could not (Fig. 19). These results suggest that nitrite is unable to upregulate mitochondrial function either directly at the level of mitochondria or upstream such as upregulated activation of AMPK (83). Interestingly, nitrite supplemenation in aged mice treated with CLA was able to rescue cardiac left ventricular dysfunction (107). This was mediated by restoring eNOS dimerization and NO signaling. These results coupled with nitrite being unable to rescue aged mitochondrial function, suggest the more prominent role of nitrite in aging is to restore NO signaling which is lost within the vasculature during aging $(107,147)$.

CLA supplementation in aged mice resulted in worsening left ventricular cardiac function (107) and decreased mitochondrial function with cLA could explain this observation. Mitochondrial function from aged mice treated with $\mathrm{CLA}$ did not result in decreased mitochondrial function (Fig. 19A-D). Therefore, the 
detrimental effects seen with cLA in aged mice is not due to diminishing cardiac mitochondrial function.

The increased oxidative and nitrative stress conditions associated with aging (147) favor the formation of nitrated fatty acids (93) and nitrated fatty acids $(46,125)$ along with precursors to fatty acid nitration (cLA and nitrite) are cardioprotective. Supplementation of $\mathrm{CLA}$ and nitrite in aged mice was unable to reverse the aged dependent decline in mitochondrial function (Fig. 14). These results suggest that a potential protective role of nitrated fatty acid or precursors to fatty acid nitration is not mediated through restoration of mitochondrial function. CLA and nitrite can upregulate HO-1 gene expression $(88,117)$ representing an alternative pathway for cardiac protection in aging.

Overall, these results demonstrate that cardiac mitochondrial function declines with age and supplementation with CLA, nitrite, or co-treatment is unable to rescue this aged related mitochondrial dysfunction. 


\section{CHAPTER VI}

\section{FUTURE DIRECTIONS AND CONCLUSIONS}

\section{Conclusion}

Cardiovascular disease is the leading cause of mortality within the United States with MI contributing the majority of morbidity. Clinical intervention to lessen cardiac injury following $\mathrm{Ml}$ is limited, highlighting a need for further research and development of new therapeutic approaches. Conjugated linoleic acid and nitrite represent dietary constituents that may be utilized to lessen cardiac injury as the combination of these dietary substances preserves cardiac function in a murine model of MI. Due to the essential role of mitochondria in maintaining normal cardiac function and recent evidence detailing a mitochondrial mechanism for nitrated fatty acids, this study was undertaken to determine how precursors to fatty acid nitration (CLA and nitrite) impacted cardiac mitochondrial function. The first part of this study revealed that a ten-day combination treatment of cLA and nitrite decreases state 3 and ETS respiration, along with complex II and III activity. The combination treatment also increased complex I activity. These results reveal that in combination, CLA and nitrite can impact cardiac mitochondrial function in vivo.

Next, cardiac mitochondrial function was assessed under two conditions

of injury or stress, MI and aging. We then assayed whether co-treatment with CLA and nitrite was able to rescue mitochondrial dysfunction during these 
conditions. In the setting of $\mathrm{Ml}$, the co-treatment was able to attenuate $\mathrm{Ml}$ induced increases in state 3 respiration, complex III activity and hydrogen peroxide levels. These results reveal a potential mitochondrial mechanism for the cardioprotection mediated by co-treatment with $\mathrm{CLA}$ and nitrite. Cardiac mitochondrial function was suppressed following aging and the combination treatment with $\mathrm{CLA}$ and nitrite was unable to restore cardiac mitochondrial function. These results reveal that the mitochondrial effects mediated by CLA and nitrite are lost in a murine model of aging. Taken together, this study demonstrates that a combination treatment of $C L A$ and nitrite is able to modulate cardiac mitochondrial function in vivo and reverse $\mathrm{MI}$, but not aging- induced mitochondrial dysfunction.

\section{Future Directions}

\section{CLA}

This study revealed that CLA was able to repress cardiac mitochondrial function and decrease complex II activity in vivo. These results suggest cLA could potentially be able to alter mitochondrial metabolism. However, the mechanism leading to this outcome is unknown. Due to the numerous upstream cellular signaling linked to $\operatorname{cLA}(101)$ that can impact mitochondrial function, it is necessary to delineate whether $\mathrm{CLA}$ is functioning upstream of mitochondria or directly at the level of mitochondria. Incubation of isolated cardiac mitochondria with CLA followed by mitochondrial respiration will reveal if CLA mediates decreases in mitochondrial respiration directly at the level of mitochondria. Co- 
incubation of CLA with etomoxir (154), an inhibitor of fatty acid beta-oxidation, on isolated cardiac mitochondria will reveal whether full length $\mathrm{CLA}$ or a downstream oxidation product is necessary to mediate $\mathrm{CLA}$ effects on mitochondria. Transport of glutamate, malate, and ADP into mitochondria is necessary for mitochondrial respiration as the isolation of mitochondria depletes endogenous substrates. The CLA mediated decrease in state 3 respiration following CLA treatment suggests transport of ADP into in mitochondria is limited. ANT-1 is responsible for the transfer of ADP into the mitochondria and ATP out of mitochondria (155). The measurement of ANT-1 activity in isolated cardiac mitochondria from mice treated with $C L A$ will be carried out using a magnesium sensitive dye, Magnesium Green. This will indicate if ANT-1 activity is decreased following CLA treatment and provide a potential mechanism by which CLA decreases mitochondrial respiration.

The mechanism for the cLA mediated decrease in complex II activity will be examined by assessing the activity of malate dehydrogenase. Malate dehydrogenase converts malate to oxaloacetate, which is a potent inhibitor of complex II activity. The cis- 9 trans-11 isomer of CLA has been shown to increase malate dehydrogenase activity in the liver of rats (156) and high levels of oxaloacetate following cLA treatment may lead to decreased complex II activity.

\section{Nitrite}

This study revealed that nitrite was able to increase complex I activity in vivo under baseline conditions. Complex I has been shown to undergo S- 
nitrosylation by an NO donor on cysteine 39 within the ND3 subunit under conditions of ischemic injury (40). This S-nitrosylation was able to transiently inhibit complex I activity and removal of this modification restored complex I activity (40). Interestingly, this modification is not seen under normoxic conditions with the NO donor. However, S-nitrosylation of cysteine 344 within the $75 \mathrm{kDa}$ subunit is found under normoxic conditions (40). Mass spectrometry analysis examing S-nitrosylation of cysteine 344 within the $75 \mathrm{kDa}$ subunit of complex I following nitrite treatment would correlate increased complex I activity with an increase in this modification; providing a link between nitrite treatment and increased complex I activity.

\section{Co-treatment of CLA and nitrite}

The major findings of this study reveal that the combination treatment of CLA and nitrite was able to decrease complex III activity under baseline conditions and attenuate $\mathrm{Ml}$ induced increases in complex III activity. Nitrated fatty acids can form adducts with free thiols via Micheal addition thereby altering protein activity (135). The cytochrome b-c1 complex subunit I, II and Rieske subunit all contain cysteiene residues that can be S-nitrosylated (134) and these residues may also undergo Micheal addition with nitrated fatty acids. Mass spectrometry analysis for nitrated CLA adducts to cysteine 69 and 410 within cytochrome b-c1 complex subunit 1, cysteine 192 within cytochrome b-c1 complex subunit 2 and cysteine 51 with cytochrome b-c1 subunit Rieske will provide insight into whether nitrated $\mathrm{CLA}$ adducts to thiols within complex III. 
Alternatively, linoleic acid is a major component of cardiolipin which is essential for complex III activity and mitochondrial supercomplex formation. Lipid peroxidation of linoleic acid within cardiolipin has been associated with decreased complex III activity and super complex formation (157). Due to structural similarities between CLA and lineolic acid, the nitration of CLA may mimic the lipid peroxidation effect of linoleic acid leading to the decreased complex III activity via supercomplex formation. The assessment of supercomplex formation by blue native PAGE following $\mathrm{CLA}$ and nitrite treatment would provide insight into mitochondrial structural architecture changes that regulate complex III activity.

Additionally, the assessment of hydrogen peroxide levels in freshly isolated mitochondria following MI would yield a stronger link between decreased complex III activity and hydrogen peroxide levels. The addition of antimycin A to isolated mitochondria that have undergone $\mathrm{MI}$ would decrease elevated complex III activity thereby lowering hydrogen peroxide levels. This would provide a mechanistic link between complex III activity and hydrogen peroxide levels following MI.

The combination treatment of $C L A$ and nitrite increases protein expression of $\mathrm{HO}-1$, and $\mathrm{HO}-1$ is a Nrf2 regulated gene. The regulation of complex III activity and hydrogen peroxide levels could potentially play a role in activating Nrf2 via redox dependent inactivation and degradation of KEAP1, the negative regulator of Nrf2. Examining nuclear translocation of Nrf2 in cardiac tissue of mice following co-treatment of CLA and nitrite would further the mechanism of $\mathrm{HO}-1$ 
mediated $\mathrm{CLA}$ and nitrite cardioprotection to include mitochondrial derived ROS via altered complex III activity.

The combination of $\operatorname{cLA}$ and nitrite could also be mediating protective against $\mathrm{Ml}$ via several alternative pathways including shifting from oxidative phosphorylation to glyolytic metabolism during $\mathrm{Ml}$ and decreasing necrosis to prevent infacrt expansion. During ischemia reperfusion injury due to the lack of oxygen cells undergo a shift in metabolism and rely on glycolysis for ATP production (158). As the combination treatment decreases complex II and III activity (Fig. 8) this could limit electron flow from the TCA cyle and beta oxidation into the electron transport chain leading to reductions in ATP. The shift to glycolysis may allow cells an apadative phase to increase ATP production via glycolysis thereby cells are more likey to survive an ischemic insult. To determine if CLA and nitrite does shift cells to a more glycolytic phenotype, the activity of phosphofructokinase will be assayed. Phosphofructokinase is the first committed step in glycolysis and would provide insight into the rate of glycolysis.

Necrosis occurs following depletion of ATP and results in cell death (159). As the combination treatment of $\operatorname{CLA}$ and nitrite could potential increase ATP production via glycolysis, this may provide a link to prevent necrosis during $\mathrm{Ml}$. By maintaining ATP levels via glycolysis during ischemia this would prevent cells from undergoing necrosis and reduce secondary inflammtory responses that occur after cell necrosis. To determine if $\mathrm{CLA}$ and nitrite is decreasing necrosis, the release of two necrosis specific markers will be assessed: peptidylprolyl 
isomerase A and non-chromatin-binding high mobility group protein B1 (160) following Ml. 


\section{REFERENCES}

1. Mozaffarian, D., E. J. Benjamin, A. S. Go, D. K. Arnett, M. J. Blaha, M. Cushman, S. de Ferranti, J. P. Despres, H. J. Fullerton, V. J. Howard, M. D. Huffman, S. E. Judd, B. M. Kissela, D. T. Lackland, J. H. Lichtman, L. D. Lisabeth, S. Liu, R. H. Mackey, D. B. Matchar, D. K. McGuire, E. R. Mohler, 3rd, C. S. Moy, P. Muntner, M. E. Mussolino, K. Nasir, R. W. Neumar, G. Nichol, L. Palaniappan, D. K. Pandey, M. J. Reeves, C. J. Rodriguez, P. D. Sorlie, J. Stein, A. Towfighi, T. N. Turan, S. S. Virani, J. Z. Willey, D. Woo, R. W. Yeh, M. B. Turner, C. American Heart Association Statistics, and S. Stroke Statistics. 2015. Heart disease and stroke statistics--2015 update: a report from the American Heart Association. Circulation 131: e29-322.

2. Ambrose, J. A., and M. Singh. 2015. Pathophysiology of coronary artery disease leading to acute coronary syndromes. F1000prime reports 7: 08.

3. Bentzon, J. F., F. Otsuka, R. Virmani, and E. Falk. 2014. Mechanisms of plaque formation and rupture. Circulation research 114: 1852-1866.

4. Quyyumi, A. A. 2003. Prognostic value of endothelial function. The American journal of cardiology 91: $19 \mathrm{H}-24 \mathrm{H}$.

5. Khan, B. V., D. G. Harrison, M. T. Olbrych, R. W. Alexander, and R. M. Medford. 1996. Nitric oxide regulates vascular cell adhesion molecule 1 gene expression and redox-sensitive transcriptional events in human vascular endothelial cells. Proceedings of the National Academy of Sciences of the United States of America 93: 9114-9119.

6. Steinberg, D., and J. L. Witztum. 2010. Oxidized low-density lipoprotein and atherosclerosis. Arteriosclerosis, thrombosis, and vascular biology 30: 23112316.

7. Skalen, K., M. Gustafsson, E. K. Rydberg, L. M. Hulten, O. Wiklund, T. L. Innerarity, and J. Boren. 2002. Subendothelial retention of atherogenic lipoproteins in early atherosclerosis. Nature 417: 750-754.

8. Srikanth, S., and J. A. Ambrose. 2012. Pathophysiology of coronary thrombus formation and adverse consequences of thrombus during PCl. Current cardiology reviews 8: 168-176. 
9. Seimon, T. A., M. J. Nadolski, X. Liao, J. Magallon, M. Nguyen, N. T. Feric, M. L. Koschinsky, R. Harkewicz, J. L. Witztum, S. Tsimikas, D. Golenbock, K. J. Moore, and I. Tabas. 2010. Atherogenic lipids and lipoproteins trigger CD36-TLR2-dependent apoptosis in macrophages undergoing endoplasmic reticulum stress. Cell metabolism 12: 467-482.

10. Virmani, R., F. D. Kolodgie, A. P. Burke, A. V. Finn, H. K. Gold, T. N. Tulenko, S. P. Wrenn, and J. Narula. 2005. Atherosclerotic plaque progression and vulnerability to rupture: angiogenesis as a source of intraplaque hemorrhage. Arteriosclerosis, thrombosis, and vascular biology 25: 2054-2061.

11. Thygesen, K., J. S. Alpert, A. S. Jaffe, M. L. Simoons, B. R. Chaitman, H. D. White, and E. S. C. A. A. H. A. W. H. F. T. F. f. t. U. D. O. M. I. Writing Group on behalf of the Joint. 2012. Third universal definition of myocardial infarction. Global heart 7: 275-295.

12. Kalogeris, T., Y. Bao, and R. J. Korthuis. 2014. Mitochondrial reactive oxygen species: a double edged sword in ischemia/reperfusion vs preconditioning. Redox Biol 2: 702-714.

13. Scarabelli, T. M., and R. A. Gottlieb. 2004. Functional and clinical repercussions of myocyte apoptosis in the multifaceted damage by ischemia/reperfusion injury: old and new concepts after 10 years of contributions. Cell death and differentiation 11 Suppl 2: S144-152.

14. Webster, K. A. 2012. Mitochondrial membrane permeabilization and cell death during myocardial infarction: roles of calcium and reactive oxygen species. Future cardiology 8: 863-884.

15. Hu, X., X. Xu, Z. Lu, P. Zhang, J. Fassett, Y. Zhang, Y. Xin, J. L. Hall, B. Viollet, R. J. Bache, Y. Huang, and Y. Chen. 2011. AMP activated protein kinasealpha2 regulates expression of estrogen-related receptor-alpha, a metabolic transcription factor related to heart failure development. Hypertension 58: 696703.

16. Sutton, M. G., and N. Sharpe. 2000. Left ventricular remodeling after myocardial infarction: pathophysiology and therapy. Circulation 101: 2981-2988.

17. Heusch, G. 2015. Molecular basis of cardioprotection: signal transduction in ischemic pre-, post-, and remote conditioning. Circulation research 116: 674699.

18. Hori, M., and K. Nishida. 2009. Oxidative stress and left ventricular remodelling after myocardial infarction. Cardiovascular research 81: 457-464.

19. Ernster, L., and G. Schatz. 1981. Mitochondria: a historical review. The Journal of cell biology $91: 227 s-255 s$. 
20. Akram, M. 2014. Citric acid cycle and role of its intermediates in metabolism. Cell biochemistry and biophysics 68: 475-478.

21. Efremov, R. G., R. Baradaran, and L. A. Sazanov. 2010. The architecture of respiratory complex I. Nature 465: 441-445.

22. Cecchini, G. 2003. Function and structure of complex II of the respiratory chain. Annual review of biochemistry 72: 77-109.

23. Crofts, A. R. 2004. The cytochrome bc1 complex: function in the context of structure. Annual review of physiology 66: 689-733.

24. Mulkidjanian, A. Y. 2005. Ubiquinol oxidation in the cytochrome bc1 complex: reaction mechanism and prevention of short-circuiting. Biochimica et biophysica acta 1709: 5-34.

25. Liu, J., L. Qin, and S. Ferguson-Miller. 2011. Crystallographic and online spectral evidence for role of conformational change and conserved water in cytochrome oxidase proton pump. Proceedings of the National Academy of Sciences of the United States of America 108: 1284-1289.

26. Kim, Y. C., and G. Hummer. 2012. Proton-pumping mechanism of cytochrome c oxidase: a kinetic master-equation approach. Biochimica et biophysica acta 1817: 526-536.

27. Nakamoto, R. K., J. A. Baylis Scanlon, and M. K. Al-Shawi. 2008. The rotary mechanism of the ATP synthase. Archives of biochemistry and biophysics 476: 43-50.

28. Lau, W. C., and J. L. Rubinstein. 2012. Subnanometre-resolution structure of the intact Thermus thermophilus H+-driven ATP synthase. Nature 481: 214218.

29. Thygesen, K., J. S. Alpert, A. S. Jaffe, M. L. Simoons, B. R. Chaitman, H. D. White, E. S. C. A. A. H. A. W. H. F. T. F. f. t. U. D. O. M. I. Joint, H. A. Katus, B. Lindahl, D. A. Morrow, P. M. Clemmensen, P. Johanson, H. Hod, R. Underwood, J. J. Bax, R. O. Bonow, F. Pinto, R. J. Gibbons, K. A. Fox, D. Atar, L. K. Newby, M. Galvani, C. W. Hamm, B. F. Uretsky, P. G. Steg, W. Wijns, J. P. Bassand, P. Menasche, J. Ravkilde, E. M. Ohman, E. M. Antman, L. C. Wallentin, P. W. Armstrong, M. L. Simoons, J. L. Januzzi, M. S. Nieminen, M. Gheorghiade, G. Filippatos, R. V. Luepker, S. P. Fortmann, W. D. Rosamond, D. Levy, D. Wood, S. C. Smith, D. Hu, J. L. Lopez-Sendon, R. M. Robertson, D. Weaver, M. Tendera, A. A. Bove, A. N. Parkhomenko, E. J. Vasilieva, and S. Mendis. 2012. Third universal definition of myocardial infarction. Circulation 126: 2020-2035. 
30. Czabotar, P. E., G. Lessene, A. Strasser, and J. M. Adams. 2014. Control of apoptosis by the BCL-2 protein family: implications for physiology and therapy. Nature reviews. Molecular cell biology 15: 49-63.

31. Elmore, S. 2007. Apoptosis: a review of programmed cell death. Toxicologic pathology 35: 495-516.

32. Ballinger, S. W. 2005. Mitochondrial dysfunction in cardiovascular disease. Free radical biology \& medicine 38: 1278-1295.

33. Schwarz, K., N. Siddiqi, S. Singh, C. J. Neil, D. K. Dawson, and M. P. Frenneaux. 2014. The breathing heart - mitochondrial respiratory chain dysfunction in cardiac disease. International journal of cardiology 171: 134-143.

34. Ide, T., H. Tsutsui, S. Hayashidani, D. Kang, N. Suematsu, K. Nakamura, H. Utsumi, N. Hamasaki, and A. Takeshita. 2001. Mitochondrial DNA damage and dysfunction associated with oxidative stress in failing hearts after myocardial infarction. Circulation research 88: 529-535.

35. Amplavanar, N. T., K. Gurpreet, M. S. Salmiah, and N. Odhayakumar. 2010. Prevalence of cardiovascular disease risk factors among attendees of the Batu 9, Cheras Health Centre, Selangor, Malaysia. The Medical journal of Malaysia 65: 173-179.

36. Tretter, L., and V. Adam-Vizi. 2004. Generation of reactive oxygen species in the reaction catalyzed by alpha-ketoglutarate dehydrogenase. The Journal of neuroscience : the official journal of the Society for Neuroscience 24: 7771-7778.

37. Quinlan, C. L., R. L. Goncalves, M. Hey-Mogensen, N. Yadava, V. I. Bunik, and M. D. Brand. 2014. The 2-oxoacid dehydrogenase complexes in mitochondria can produce superoxide/hydrogen peroxide at much higher rates than complex I. The Journal of biological chemistry 289: 8312-8325.

38. Chen, Y. R., and J. L. Zweier. 2014. Cardiac mitochondria and reactive oxygen species generation. Circulation research 114: 524-537.

39. Wang, P., H. Chen, H. Qin, S. Sankarapandi, M. W. Becher, P. C. Wong, and J. L. Zweier. 1998. Overexpression of human copper, zinc-superoxide dismutase (SOD1) prevents postischemic injury. Proceedings of the National Academy of Sciences of the United States of America 95: 4556-4560.

40. Chouchani, E. T., C. Methner, S. M. Nadtochiy, A. Logan, V. R. Pell, S. Ding, A. M. James, H. M. Cocheme, J. Reinhold, K. S. Lilley, L. Partridge, I. M. Fearnley, A. J. Robinson, R. C. Hartley, R. A. Smith, T. Krieg, P. S. Brookes, and M. P. Murphy. 2013. Cardioprotection by S-nitrosation of a cysteine switch on mitochondrial complex I. Nature medicine 19: 753-759. 
41. Murry, C. E., R. B. Jennings, and K. A. Reimer. 1986. Preconditioning with ischemia: a delay of lethal cell injury in ischemic myocardium. Circulation 74: 1124-1136.

42. Wojtovich, A. P., S. M. Nadtochiy, P. S. Brookes, and K. Nehrke. 2012. Ischemic preconditioning: the role of mitochondria and aging. Experimental gerontology 47: 1-7.

43. Ertracht, O., A. Malka, S. Atar, and O. Binah. 2014. The mitochondria as a target for cardioprotection in acute myocardial ischemia. Pharmacology \& therapeutics 142: 33-40.

44. Chen, Y., J. Liu, Y. Zheng, J. Wang, Z. Wang, S. Gu, J. Tan, Q. Jing, and $\mathrm{H}$. Yang. 2015. Uncoupling protein 3 mediates $\mathrm{H}(2) \mathrm{O}(2)$ preconditioning-afforded cardioprotection through the inhibition of MPTP opening. Cardiovascular research 105: 192-202.

45. McLeod, C. J., A. Aziz, R. F. Hoyt, Jr., J. P. McCoy, Jr., and M. N. Sack. 2005. Uncoupling proteins 2 and 3 function in concert to augment tolerance to cardiac ischemia. The Journal of biological chemistry 280: 33470-33476.

46. Nadtochiy, S. M., Q. Zhu, W. Urciuoli, R. Rafikov, S. M. Black, and P. S. Brookes. 2012. Nitroalkenes confer acute cardioprotection via adenine nucleotide translocase 1. The Journal of biological chemistry 287: 3573-3580.

47. Nadtochiy, S. M., P. R. Baker, B. A. Freeman, and P. S. Brookes. 2009. Mitochondrial nitroalkene formation and mild uncoupling in ischaemic preconditioning: implications for cardioprotection. Cardiovascular research $\mathbf{8 2}$ : 333-340.

48. Chen, Q., M. Paillard, L. Gomez, H. Li, Y. Hu, and E. J. Lesnefsky. 2012. Postconditioning modulates ischemia-damaged mitochondria during reperfusion. Journal of cardiovascular pharmacology 59: 101-108.

49. Lundberg, J. O., M. T. Gladwin, A. Ahluwalia, N. Benjamin, N. S. Bryan, A. Butler, P. Cabrales, A. Fago, M. Feelisch, P. C. Ford, B. A. Freeman, M. Frenneaux, J. Friedman, M. Kelm, C. G. Kevil, D. B. Kim-Shapiro, A. V. Kozlov, J. R. Lancaster, Jr., D. J. Lefer, K. McColl, K. McCurry, R. P. Patel, J. Petersson, T. Rassaf, V. P. Reutov, G. B. Richter-Addo, A. Schechter, S. Shiva, K. Tsuchiya, E. E. van Faassen, A. J. Webb, B. S. Zuckerbraun, J. L. Zweier, and E. Weitzberg. 2009. Nitrate and nitrite in biology, nutrition and therapeutics. Nature chemical biology 5: 865-869.

50. Furchgott, R. F., and S. Bhadrakom. 1953. Reactions of strips of rabbit aorta to epinephrine, isopropylarterenol, sodium nitrite and other drugs. The Journal of pharmacology and experimental therapeutics 108: 129-143. 
51. Shiva, S., M. N. Sack, J. J. Greer, M. Duranski, L. A. Ringwood, L. Burwell, X. Wang, P. H. MacArthur, A. Shoja, N. Raghavachari, J. W. Calvert, P. S. Brookes, D. J. Lefer, and M. T. Gladwin. 2007. Nitrite augments tolerance to ischemia/reperfusion injury via the modulation of mitochondrial electron transfer. The Journal of experimental medicine 204: 2089-2102.

52. Lundberg, J. O., M. T. Gladwin, and E. Weitzberg. 2015. Strategies to increase nitric oxide signalling in cardiovascular disease. Nature reviews. Drug discovery 14: 623-641.

53. Andrew, P. J., and B. Mayer. 1999. Enzymatic function of nitric oxide synthases. Cardiovascular research 43: 521-531.

54. Bian, K., and F. Murad. 2007. Nitric oxide signaling in vascular biology. Journal of the American Society of Hypertension : JASH 1: 17-29.

55. Lundberg, J. O., E. Weitzberg, J. A. Cole, and N. Benjamin. 2004. Nitrate, bacteria and human health. Nature reviews. Microbiology 2: 593-602.

56. Hobbs, A. J. 1997. Soluble guanylate cyclase: the forgotten sibling. Trends in pharmacological sciences 18: 484-491.

57. Cleeter, M. W., J. M. Cooper, V. M. Darley-Usmar, S. Moncada, and A. H. Schapira. 1994. Reversible inhibition of cytochrome c oxidase, the terminal enzyme of the mitochondrial respiratory chain, by nitric oxide. Implications for neurodegenerative diseases. FEBS letters 345: 50-54.

58. De Pascali, F., C. Hemann, K. Samons, C. A. Chen, and J. L. Zweier. 2014. Hypoxia and Reoxygenation Induce Endothelial Nitric Oxide Synthase Uncoupling in Endothelial Cells through Tetrahydrobiopterin Depletion and SGlutathionylation. Biochemistry 53: 3679-3688.

59. Sasaki, N., T. Sato, A. Ohler, B. O'Rourke, and E. Marban. 2000. Activation of mitochondrial ATP-dependent potassium channels by nitric oxide. Circulation 101: 439-445.

60. Ertracht, O., A. Malka, S. Atar, and O. Binah. 2014. The mitochondria as a target for cardioprotection in acute myocardial ischemia. Pharmacology \& therapeutics 142: 33-40.

61. Lundberg, J. O., and E. Weitzberg. 2013. Biology of nitrogen oxides in the gastrointestinal tract. Gut 62: 616-629.

62. Cosby, K., K. S. Partovi, J. H. Crawford, R. P. Patel, C. D. Reiter, S. Martyr, B. K. Yang, M. A. Waclawiw, G. Zalos, X. Xu, K. T. Huang, H. Shields, D. B. Kim-Shapiro, A. N. Schechter, R. O. Cannon, 3rd, and M. T. Gladwin. 2003. 
Nitrite reduction to nitric oxide by deoxyhemoglobin vasodilates the human circulation. Nature medicine 9: 1498-1505.

63. Totzeck, M., U. B. Hendgen-Cotta, P. Luedike, M. Berenbrink, J. P. Klare, H. J. Steinhoff, D. Semmler, S. Shiva, D. Williams, A. Kipar, M. T. Gladwin, J. Schrader, M. Kelm, A. R. Cossins, and T. Rassaf. 2012. Nitrite regulates hypoxic vasodilation via myoglobin-dependent nitric oxide generation. Circulation 126: 325-334.

64. Jayaraman, T., J. Tejero, B. B. Chen, A. B. Blood, S. Frizzell, C. Shapiro, M. Tiso, B. L. Hood, X. Wang, X. Zhao, T. P. Conrads, R. K. Mallampalli, and M. T. Gladwin. 2011. 14-3-3 binding and phosphorylation of neuroglobin during hypoxia modulate six-to-five heme pocket coordination and rate of nitrite reduction to nitric oxide. The Journal of biological chemistry 286: 42679-42689.

65. Basu, S., N. A. Azarova, M. D. Font, S. B. King, N. Hogg, M. T. Gladwin, S. Shiva, and D. B. Kim-Shapiro. 2008. Nitrite reductase activity of cytochrome C. The Journal of biological chemistry 283: 32590-32597.

66. Castello, P. R., P. S. David, T. McClure, Z. Crook, and R. O. Poyton. 2006. Mitochondrial cytochrome oxidase produces nitric oxide under hypoxic conditions: implications for oxygen sensing and hypoxic signaling in eukaryotes. Cell metabolism 3: 277-287.

67. Gautier, C., E. van Faassen, I. Mikula, P. Martasek, and A. SlamaSchwok. 2006. Endothelial nitric oxide synthase reduces nitrite anions to NO under anoxia. Biochemical and biophysical research communications 341: 816821.

68. Li, H., A. Samouilov, X. Liu, and J. L. Zweier. 2001. Characterization of the magnitude and kinetics of xanthine oxidase-catalyzed nitrite reduction. Evaluation of its role in nitric oxide generation in anoxic tissues. The Journal of biological chemistry 276: 24482-24489.

69. Crawford, J. H., T. S. Isbell, Z. Huang, S. Shiva, B. K. Chacko, A. N. Schechter, V. M. Darley-Usmar, J. D. Kerby, J. D. Lang, Jr., D. Kraus, C. Ho, M. T. Gladwin, and R. P. Patel. 2006. Hypoxia, red blood cells, and nitrite regulate NO-dependent hypoxic vasodilation. Blood 107: 566-574.

70. Nystrom, T., H. Ortsater, Z. Huang, F. Zhang, F. J. Larsen, E. Weitzberg, J. O. Lundberg, and A. Sjoholm. 2012. Inorganic nitrite stimulates pancreatic islet blood flow and insulin secretion. Free radical biology \& medicine 53: 1017-1023.

71. Baker, J. E., J. Su, X. Fu, A. Hsu, G. J. Gross, J. S. Tweddell, and N. Hogg. 2007. Nitrite confers protection against myocardial infarction: role of 
xanthine oxidoreductase, NADPH oxidase and K(ATP) channels. Journal of molecular and cellular cardiology 43: 437-444.

72. Bryan, N. S., J. W. Calvert, S. Gundewar, and D. J. Lefer. 2008. Dietary nitrite restores NO homeostasis and is cardioprotective in endothelial nitric oxide synthase-deficient mice. Free radical biology \& medicine 45: 468-474.

73. Shiva, S., and M. T. Gladwin. 2009. Nitrite mediates cytoprotection after ischemia/reperfusion by modulating mitochondrial function. Basic research in cardiology 104: 113-119.

74. Webb, A., R. Bond, P. McLean, R. Uppal, N. Benjamin, and A. Ahluwalia. 2004. Reduction of nitrite to nitric oxide during ischemia protects against myocardial ischemia-reperfusion damage. Proceedings of the National Academy of Sciences of the United States of America 101: 13683-13688.

75. Hendgen-Cotta, U. B., M. W. Merx, S. Shiva, J. Schmitz, S. Becher, J. P. Klare, H. J. Steinhoff, A. Goedecke, J. Schrader, M. T. Gladwin, M. Kelm, and T. Rassaf. 2008. Nitrite reductase activity of myoglobin regulates respiration and cellular viability in myocardial ischemia-reperfusion injury. Proceedings of the National Academy of Sciences of the United States of America 105: 1025610261.

76. Duranski, M. R., J. J. Greer, A. Dejam, S. Jaganmohan, N. Hogg, W. Langston, R. P. Patel, S. F. Yet, X. Wang, C. G. Kevil, M. T. Gladwin, and D. J. Lefer. 2005. Cytoprotective effects of nitrite during in vivo ischemia-reperfusion of the heart and liver. The Journal of clinical investigation 115: 1232-1240.

77. Jung, K. H., K. Chu, S. Y. Ko, S. T. Lee, D. I. Sinn, D. K. Park, J. M. Kim, E. C. Song, M. Kim, and J. K. Roh. 2006. Early intravenous infusion of sodium nitrite protects brain against in vivo ischemia-reperfusion injury. Stroke; a journal of cerebral circulation 37: 2744-2750.

78. Hendgen-Cotta, U. B., P. Luedike, M. Totzeck, M. Kropp, A. Schicho, P. Stock, C. Rammos, M. Niessen, C. Heiss, J. O. Lundberg, E. Weitzberg, M. Kelm, and T. Rassaf. 2012. Dietary nitrate supplementation improves revascularization in chronic ischemia. Circulation 126: 1983-1992.

79. Bolli, R. 2001. Cardioprotective function of inducible nitric oxide synthase and role of nitric oxide in myocardial ischemia and preconditioning: an overview of a decade of research. Journal of molecular and cellular cardiology 33: 18971918.

80. Kozlov, A. V., K. Staniek, and H. Nohl. 1999. Nitrite reductase activity is a novel function of mammalian mitochondria. FEBS letters 454: 127-130. 
81. Brookes, P. S., S. Shiva, R. P. Patel, and V. M. Darley-Usmar. 2002. Measurement of mitochondrial respiratory thresholds and the control of respiration by nitric oxide. Methods in enzymology 359: 305-319.

82. Mo, L., Y. Wang, L. Geary, C. Corey, M. J. Alef, D. Beer-Stolz, B. S. Zuckerbraun, and S. Shiva. 2012. Nitrite activates AMP kinase to stimulate mitochondrial biogenesis independent of soluble guanylate cyclase. Free radical biology \& medicine 53: 1440-1450.

83. Khoo, N. K., L. Mo, S. Zharikov, C. Kamga-Pride, K. Quesnelle, F. GolinBisello, L. Li, Y. Wang, and S. Shiva. 2014. Nitrite augments glucose uptake in adipocytes through the protein kinase A-dependent stimulation of mitochondrial fusion. Free radical biology \& medicine 70: 45-53.

84. Kamga Pride, C., L. Mo, K. Quesnelle, R. K. Dagda, D. Murillo, L. Geary, C. Corey, R. Portella, S. Zharikov, C. St Croix, S. Maniar, C. T. Chu, N. K. Khoo, and S. Shiva. 2014. Nitrite activates protein kinase A in normoxia to mediate mitochondrial fusion and tolerance to ischaemia/reperfusion. Cardiovascular research 101: 57-68.

85. Xu, A., K. Szczepanek, M. W. Maceyka, T. Ross, E. Bowler, Y. Hu, B. Kenny, C. Mehfoud, P. N. Desai, C. M. Baumgarten, Q. Chen, and E. J. Lesnefsky. 2014. Transient complex I inhibition at the onset of reperfusion by extracellular acidification decreases cardiac injury. American journal of physiology. Cell physiology 306: C1142-1153.

86. Vlasova, II, V. A. Tyurin, A. A. Kapralov, I. V. Kurnikov, A. N. Osipov, M. V. Potapovich, D. A. Stoyanovsky, and V. E. Kagan. 2006. Nitric oxide inhibits peroxidase activity of cytochrome c.cardiolipin complex and blocks cardiolipin oxidation. The Journal of biological chemistry 281: 14554-14562.

87. Larsen, F. J., T. A. Schiffer, S. Borniquel, K. Sahlin, B. Ekblom, J. O. Lundberg, and E. Weitzberg. 2011. Dietary inorganic nitrate improves mitochondrial efficiency in humans. Cell metabolism 13: 149-159.

88. Qipshidze-Kelm, N., K. M. Piell, J. C. Solinger, and M. P. Cole. 2013. Cotreatment with conjugated linoleic acid and nitrite protects against myocardial infarction. Redox Biol 2: 1-7.

89. Kim, A. S., E. J. Miller, T. M. Wright, J. Li, D. Qi, K. Atsina, V. Zaha, K. Sakamoto, and L. H. Young. 2011. A small molecule AMPK activator protects the heart against ischemia-reperfusion injury. Journal of molecular and cellular cardiology 51: 24-32.

90. Jones, D. A., M. Andiapen, T. J. Van-Eijl, A. J. Webb, S. Antoniou, R. J. Schilling, A. Ahluwalia, and A. Mathur. 2013. The safety and efficacy of 
intracoronary nitrite infusion during acute myocardial infarction (NITRITE-AMI): study protocol of a randomised controlled trial. BMJ open 3.

91. Siddiqi, N., C. Neil, M. Bruce, G. MacLennan, S. Cotton, S.

Papadopoulou, M. Feelisch, N. Bunce, P. O. Lim, D. Hildick-Smith, J. Horowitz, M. Madhani, N. Boon, D. Dawson, J. C. Kaski, M. Frenneaux, and N. investigators. 2014. Intravenous sodium nitrite in acute ST-elevation myocardial infarction: a randomized controlled trial (NIAMI). European heart journal 35: 1255-1262.

92. Batthyany, C., F. J. Schopfer, P. R. Baker, R. Duran, L. M. Baker, Y. Huang, C. Cervenansky, B. P. Branchaud, and B. A. Freeman. 2006. Reversible post-translational modification of proteins by nitrated fatty acids in vivo. The Journal of biological chemistry 281: 20450-20463.

93. Freeman, B. A., P. R. Baker, F. J. Schopfer, S. R. Woodcock, A. Napolitano, and M. d'Ischia. 2008. Nitro-fatty acid formation and signaling. The Journal of biological chemistry 283: 15515-15519.

94. Yang, B., H. Q. Chen, C. Stanton, R. P. Ross, H. Zhang, Y. Q. Chen, and W. Chen. 2015. Review of the roles of conjugated linoleic acid in health and disease. J Funct Foods 15: 314-325.

95. Madron, M. S., D. G. Peterson, D. A. Dwyer, B. A. Corl, L. H. Baumgard, D. H. Beermann, and D. E. Bauman. 2002. Effect of extruded full-fat soybeans on conjugated linoleic acid content of intramuscular, intermuscular, and subcutaneous fat in beef steers. Journal of animal science 80: 1135-1143.

96. Chin, S. F., J. M. Storkson, W. Liu, and M. W. Pariza. 1991. Dietary Sources of the Anticarcinogen Cla (Conjugated Dienoic Derivatives of LinoleicAcid). Faseb J 5: A1444-A1444.

97. Dhiman, T. R., S. H. Nam, and A. L. Ure. 2005. Factors affecting conjugated linoleic acid content in milk and meat. Critical reviews in food science and nutrition 45: 463-482.

98. Ritzenthaler, K. L., M. K. McGuire, R. Falen, T. D. Shultz, N. Dasgupta, and M. A. McGuire. 2001. Estimation of conjugated linoleic acid intake by written dietary assessment methodologies underestimates actual intake evaluated by food duplicate methodology. The Journal of nutrition 131: 1548-1554.

99. Food, and H. H. S. Drug Administration. 2003. Food labeling: trans fatty acids in nutrition labeling, nutrient content claims, and health claims. Final rule. Federal register 68: 41433-41506. 
100. Belury, M. A. 2002. Dietary conjugated linoleic acid in health: physiological effects and mechanisms of action. Annual review of nutrition 22: 505-531.

101. Gebauer, S. K., J. M. Chardigny, M. U. Jakobsen, B. Lamarche, A. L. Lock, S. D. Proctor, and D. J. Baer. 2011. Effects of ruminant trans fatty acids on cardiovascular disease and cancer: a comprehensive review of epidemiological, clinical, and mechanistic studies. Advances in nutrition 2: 332-354.

102. Smit, L. A., A. Baylin, and H. Campos. 2010. Conjugated linoleic acid in adipose tissue and risk of myocardial infarction. The American journal of clinical nutrition 92: 34-40.

103. Varga, T., Z. Czimmerer, and L. Nagy. 2011. PPARs are a unique set of fatty acid regulated transcription factors controlling both lipid metabolism and inflammation. Biochimica et biophysica acta 1812: 1007-1022.

104. Riserus, U., B. Vessby, J. Arnlov, and S. Basu. 2004. Effects of cis9,trans-11 conjugated linoleic acid supplementation on insulin sensitivity, lipid peroxidation, and proinflammatory markers in obese men. The American journal of clinical nutrition 80: 279-283.

105. Stachowska, E., M. Baskiewicz-Masiuk, V. Dziedziejko, I. Gutowska, I. Baranowska-Bosiacka, M. Marchlewicz, B. Dolegowska, B. Wiszniewska, B. Machalinski, and D. Chlubek. 2008. Conjugated linoleic acid increases intracellular ROS synthesis and oxygenation of arachidonic acid in macrophages. Nutrition 24: 187-199.

106. Diniz, Y. S., P. P. Santos, H. B. Assalin, G. A. Souza, K. K. Rocha, G. M. Ebaid, F. R. Seiva, J. F. Amauchi, J. L. Novelli Filho, and E. L. Novelli. 2008. Conjugated linoleic acid and cardiac health: oxidative stress and energetic metabolism in standard and sucrose-rich diets. European journal of pharmacology 579: 318-325.

107. Piell, K. M., N. Qipshidze Kelm, M. P. Caroway, M. Aman, and M. P. Cole. 2014. Nitrite treatment rescues cardiac dysfunction in aged mice treated with conjugated linoleic acid. Free radical biology \& medicine 72C: 66-75.

108. Tricon, S., G. C. Burdge, S. Kew, T. Banerjee, J. J. Russell, E. L. Jones, R. F. Grimble, C. M. Williams, P. Yaqoob, and P. C. Calder. 2004. Opposing effects of cis-9,trans-11 and trans-10,cis-12 conjugated linoleic acid on blood lipids in healthy humans. The American journal of clinical nutrition 80: 614-620.

109. Kritchevsky, D., S. A. Tepper, S. Wright, S. K. Czarnecki, T. A. Wilson, and R. J. Nicolosi. 2004. Conjugated linoleic acid isomer effects in atherosclerosis: growth and regression of lesions. Lipids 39: 611-616. 
110. LeDoux, M., L. Laloux, J. J. Fontaine, Y. A. Carpentier, J. M. Chardigny, and J. L. Sebedio. 2007. Rumenic acid significantly reduces plasma levels of LDL and small dense LDL cholesterol in hamsters fed a cholesterol- and lipidenriched semi-purified diet. Lipids 42: 135-141.

111. Wilson, T. A., R. J. Nicolosi, A. Saati, T. Kotyla, and D. Kritchevsky. 2006. Conjugated linoleic acid isomers reduce blood cholesterol levels but not aortic cholesterol accumulation in hypercholesterolemic hamsters. Lipids 41: 41-48.

112. Moya-Camarena, S. Y., J. P. Vanden Heuvel, S. G. Blanchard, L. A. Leesnitzer, and M. A. Belury. 1999. Conjugated linoleic acid is a potent naturally occurring ligand and activator of PPARalpha. Journal of lipid research 40: 14261433.

113. Kim, Y., and Y. Park. 2015. Conjugated linoleic acid (CLA) stimulates mitochondrial biogenesis signaling by the upregulation of PPARgamma coactivator 1alpha (PGC-1alpha) in C2C12 cells. Lipids 50: 329-338.

114. Peters, J. M., Y. Park, F. J. Gonzalez, and M. W. Pariza. 2001. Influence of conjugated linoleic acid on body composition and target gene expression in peroxisome proliferator-activated receptor alpha-null mice. Biochimica et biophysica acta 1533: 233-242.

115. Demizieux, L., P. Degrace, J. Gresti, O. Loreau, J. P. Noel, J. M. Chardigny, J. L. Sebedio, and P. Clouet. 2002. Conjugated linoleic acid isomers in mitochondria: evidence for an alteration of fatty acid oxidation. Journal of lipid research 43: 2112-2122.

116. Ferramosca, A., V. Savy, L. Conte, S. Colombo, A. W. Einerhand, and V. Zara. 2006. Conjugated linoleic acid and hepatic lipogenesis in mouse: role of the mitochondrial citrate carrier. Journal of lipid research 47: 1994-2003.

117. Bonacci, G., P. R. S. Baker, S. R. Salvatore, D. Shores, N. K. H. Khoo, J. R. Koenitzer, D. A. Vitturi, S. R. Woodcock, F. Golin-Bisello, M. P. Cole, S. Watkins, C. St. Croix, C. I. Batthyany, B. A. Freeman, and F. J. Schopfer. 2012. Conjugated Linoleic Acid Is a Preferential Substrate for Fatty Acid Nitration. Journal of Biological Chemistry 287: 44071-44082.

118. Trostchansky, A., L. Bonilla, L. Gonzalez-Perilli, and H. Rubbo. 2013. Nitro-fatty acids: formation, redox signaling, and therapeutic potential. Antioxidants \& redox signaling 19: 1257-1265.

119. Forman, B. M., P. Tontonoz, J. Chen, R. P. Brun, B. M. Spiegelman, and R. M. Evans. 1995. 15-Deoxy-delta 12, 14-prostaglandin J2 is a ligand for the adipocyte determination factor PPAR gamma. Cell 83: 803-812. 
120. Rubbo, H., R. Radi, M. Trujillo, R. Telleri, B. Kalyanaraman, S. Barnes, M. Kirk, and B. A. Freeman. 1994. Nitric oxide regulation of superoxide and peroxynitrite-dependent lipid peroxidation. Formation of novel nitrogen-containing oxidized lipid derivatives. The Journal of biological chemistry 269: 26066-26075.

121. Baker, P. R., Y. Lin, F. J. Schopfer, S. R. Woodcock, A. L. Groeger, C. Batthyany, S. Sweeney, M. H. Long, K. E. Iles, L. M. Baker, B. P. Branchaud, Y. E. Chen, and B. A. Freeman. 2005. Fatty acid transduction of nitric oxide signaling: multiple nitrated unsaturated fatty acid derivatives exist in human blood and urine and serve as endogenous peroxisome proliferator-activated receptor ligands. The Journal of biological chemistry 280: 42464-42475.

122. Levonen, A. L., A. Landar, A. Ramachandran, E. K. Ceaser, D. A. Dickinson, G. Zanoni, J. D. Morrow, and V. M. Darley-Usmar. 2004. Cellular mechanisms of redox cell signalling: role of cysteine modification in controlling antioxidant defences in response to electrophilic lipid oxidation products. The Biochemical journal 378: 373-382.

123. Khoo, N. K., V. Rudolph, M. P. Cole, F. Golin-Bisello, F. J. Schopfer, S. R. Woodcock, C. Batthyany, and B. A. Freeman. 2010. Activation of vascular endothelial nitric oxide synthase and heme oxygenase-1 expression by electrophilic nitro-fatty acids. Free radical biology \& medicine 48: 230-239.

124. Cui, T., F. J. Schopfer, J. Zhang, K. Chen, T. Ichikawa, P. R. Baker, C. Batthyany, B. K. Chacko, X. Feng, R. P. Patel, A. Agarwal, B. A. Freeman, and Y. E. Chen. 2006. Nitrated fatty acids: Endogenous anti-inflammatory signaling mediators. The Journal of biological chemistry 281: 35686-35698.

125. Rudolph, V., T. K. Rudolph, F. J. Schopfer, G. Bonacci, S. R. Woodcock, M. P. Cole, P. R. Baker, R. Ramani, and B. A. Freeman. 2010. Endogenous generation and protective effects of nitro-fatty acids in a murine model of focal cardiac ischaemia and reperfusion. Cardiovascular research 85: 155-166.

126. Nadtochiy, S. M., J. Madukwe, F. Hagen, and P. S. Brookes. 2014. Mitochondrially targeted nitro-linoleate: a new tool for the study of cardioprotection. British journal of pharmacology 171: 2091-2098.

127. Delmastro-Greenwood, M., K. S. Hughan, D. A. Vitturi, S. R. Salvatore, G. Grimes, G. Potti, S. Shiva, F. J. Schopfer, M. T. Gladwin, B. A. Freeman, and S. Gelhaus Wendell. 2015. Nitrite and nitrate-dependent generation of antiinflammatory fatty acid nitroalkenes. Free radical biology \& medicine.

128. Long, J., J. Ma, C. Luo, X. Mo, L. Sun, W. Zang, and J. Liu. 2009. Comparison of two methods for assaying complex I activity in mitochondria isolated from rat liver, brain and heart. Life sciences 85: 276-280. 
129. Spinazzi, M., A. Casarin, V. Pertegato, L. Salviati, and C. Angelini. 2012. Assessment of mitochondrial respiratory chain enzymatic activities on tissues and cultured cells. Nat Protoc 7: 1235-1246.

130. Ryder, J. W., C. P. Portocarrero, X. M. Song, L. Cui, M. Yu, T. Combatsiaris, D. Galuska, D. E. Bauman, D. M. Barbano, M. J. Charron, J. R. Zierath, and K. L. Houseknecht. 2001. Isomer-specific antidiabetic properties of conjugated linoleic acid. Improved glucose tolerance, skeletal muscle insulin action, and UCP-2 gene expression. Diabetes 50: 1149-1157.

131. Borniquel, S., C. Jadert, and J. O. Lundberg. 2012. Dietary conjugated linoleic acid activates PPARgamma and the intestinal trefoil factor in SW480 cells and mice with dextran sulfate sodium-induced colitis. The Journal of nutrition 142: $2135-2140$.

132. Moloney, F., T. P. Yeow, A. Mullen, J. J. Nolan, and H. M. Roche. 2004. Conjugated linoleic acid supplementation, insulin sensitivity, and lipoprotein metabolism in patients with type 2 diabetes mellitus. The American journal of clinical nutrition 80: 887-895.

133. Vaughan, R. A., R. Garcia-Smith, M. Bisoffi, C. A. Conn, and K. A. Trujillo. 2012. Conjugated linoleic acid or omega 3 fatty acids increase mitochondrial biosynthesis and metabolism in skeletal muscle cells. Lipids in health and disease 11: 142.

134. Doulias, P. T., M. Tenopoulou, J. L. Greene, K. Raju, and H. Ischiropoulos. 2013. Nitric oxide regulates mitochondrial fatty acid metabolism through reversible protein S-nitrosylation. Science signaling 6: rs1.

135. Baker, L. M., P. R. Baker, F. Golin-Bisello, F. J. Schopfer, M. Fink, S. R. Woodcock, B. P. Branchaud, R. Radi, and B. A. Freeman. 2007. Nitro-fatty acid reaction with glutathione and cysteine. Kinetic analysis of thiol alkylation by a Michael addition reaction. The Journal of biological chemistry 282: 31085-31093.

136. Krijnen, P. A., R. Nijmeijer, C. J. Meijer, C. A. Visser, C. E. Hack, and H. W. Niessen. 2002. Apoptosis in myocardial ischaemia and infarction. Journal of clinical pathology 55: 801-811.

137. Mateus, P. S., C. C. Dias, N. Betrencourt, L. Adao, L. Santos, F. Sampaio, C. Mateus, J. Primo, L. Simoes, H. Barros, and V. G. Ribeiro. 2005. Left ventricular dysfunction after acute myocardial infarction--the impact of cardiovascular risk factors. Revista portuguesa de cardiologia : orgao oficial da Sociedade Portuguesa de Cardiologia = Portuguese journal of cardiology : an official journal of the Portuguese Society of Cardiology 24: 727-734. 
138. Nunnari, J., and A. Suomalainen. 2012. Mitochondria: in sickness and in health. Cell 148: 1145-1159.

139. Renner, A., M. R. Sagstetter, H. Harms, V. Lange, M. E. Gotz, and O. Elert. 2005. Formation of 4-hydroxy-2-nonenal protein adducts in the ischemic rat heart after transplantation. The Journal of heart and lung transplantation : the official publication of the International Society for Heart Transplantation 24: 730736.

140. Boengler, K., D. Hilfiker-Kleiner, G. Heusch, and R. Schulz. 2010. Inhibition of permeability transition pore opening by mitochondrial STAT3 and its role in myocardial ischemia/reperfusion. Basic research in cardiology 105: 771785.

141. Bhamra, G. S., D. J. Hausenloy, S. M. Davidson, R. D. Carr, M. Paiva, A. M. Wynne, M. M. Mocanu, and D. M. Yellon. 2008. Metformin protects the ischemic heart by the Akt-mediated inhibition of mitochondrial permeability transition pore opening. Basic research in cardiology 103: 274-284.

142. Brennan, J. P., R. Southworth, R. A. Medina, S. M. Davidson, M. R. Duchen, and M. J. Shattock. 2006. Mitochondrial uncoupling, with low concentration FCCP, induces ROS-dependent cardioprotection independent of KATP channel activation. Cardiovascular research 72: 313-321.

143. Methner, C., E. T. Chouchani, G. Buonincontri, V. R. Pell, S. J. Sawiak, M. P. Murphy, and T. Krieg. 2014. Mitochondria selective S-nitrosation by mitochondria-targeted S-nitrosothiol protects against post-infarct heart failure in mouse hearts. European journal of heart failure 16: 712-717.

144. Murphy, M. P. 2009. How mitochondria produce reactive oxygen species. The Biochemical journal 417: 1-13.

145. Zweier, J. L., A. Samouilov, and P. Kuppusamy. 1999. Non-enzymatic nitric oxide synthesis in biological systems. Biochimica et biophysica acta 1411: 250-262.

146. Shih, H., B. Lee, R. J. Lee, and A. J. Boyle. 2011. The aging heart and post-infarction left ventricular remodeling. Journal of the American College of Cardiology 57: 9-17.

147. Mikhed, Y., A. Daiber, and S. Steven. 2015. Mitochondrial Oxidative Stress, Mitochondrial DNA Damage and Their Role in Age-Related Vascular Dysfunction. International journal of molecular sciences 16: 15918-15953. 
148. Kwong, L. K., and R. S. Sohal. 2000. Age-related changes in activities of mitochondrial electron transport complexes in various tissues of the mouse. Archives of biochemistry and biophysics 373: 16-22.

149. Fannin, S. W., E. J. Lesnefsky, T. J. Slabe, M. O. Hassan, and C. L. Hoppel. 1999. Aging selectively decreases oxidative capacity in rat heart interfibrillar mitochondria. Archives of biochemistry and biophysics 372: 399-407.

150. Lenaz, G., C. Bovina, C. Castelluccio, R. Fato, G. Formiggini, M. L. Genova, M. Marchetti, M. M. Pich, F. Pallotti, G. Parenti Castelli, and G. Biagini. 1997. Mitochondrial complex I defects in aging. Molecular and cellular biochemistry 174: 329-333.

151. Paneni, F., S. Costantino, and F. Cosentino. 2015. Molecular pathways of arterial aging. Clinical science 128: 69-79.

152. Juhaszova, M., C. Rabuel, D. B. Zorov, E. G. Lakatta, and S. J. Sollott. 2005. Protection in the aged heart: preventing the heart-break of old age? Cardiovascular research 66: 233-244.

153. Ferrara, N., B. Rinaldi, G. Corbi, V. Conti, P. Stiuso, S. Boccuti, G. Rengo, F. Rossi, and A. Filippelli. 2008. Exercise training promotes SIRT1 activity in aged rats. Rejuvenation research 11: 139-150.

154. Horn, C. C., H. Ji, and M. I. Friedman. 2004. Etomoxir, a fatty acid oxidation inhibitor, increases food intake and reduces hepatic energy status in rats. Physiology \& behavior 81: 157-162.

155. Sharer, J. D. 2005. The adenine nucleotide translocase type 1 (ANT1): a new factor in mitochondrial disease. IUBMB life 57: 607-614.

156. Faulconnier, Y., M. A. Arnal, P. Patureau Mirand, J. M. Chardigny, and Y. Chilliard. 2004. Isomers of conjugated linoleic acid decrease plasma lipids and stimulate adipose tissue lipogenesis without changing adipose weight in postprandial adult sedentary or trained Wistar rat. The Journal of nutritional biochemistry 15: 741-748.

157. Paradies, G., V. Paradies, V. De Benedictis, F. M. Ruggiero, and G. Petrosillo. 2014. Functional role of cardiolipin in mitochondrial bioenergetics. Biochimica et biophysica acta 1837: 408-417.

158. Jaswal, J. S., W. Keung, W. Wang, J. R. Ussher, and G. D. Lopaschuk. 2011. Targeting fatty acid and carbohydrate oxidation--a novel therapeutic intervention in the ischemic and failing heart. Biochimica et biophysica acta 1813: 1333-1350. 
159. Golstein, P., and G. Kroemer. 2007. Cell death by necrosis: towards a molecular definition. Trends in biochemical sciences 32: 37-43.

160. Kepp, O., L. Galluzzi, M. Lipinski, J. Yuan, and G. Kroemer. 2011. Cell death assays for drug discovery. Nature reviews. Drug discovery 10: 221-237. 


\section{CURRICULUM VITA}

NAME:

ADDRESS:

DOB:

EDUCATION

\& TRAINING:
Patrick Van Hoose

2139 Lexington Rd. \#331

Louisville, KY, 40206

Louisville, Kentucky - September 11, 1989
B.S., Biology

Spring Hill College

2007-2011

Research Assistant

University of Louisville, Dept. of Molecular Cardiology

June 2010- August 2010

Research Assistant

University of Louisville, Dept. of Molecular Cardiology

May 2011- August 2011

Ph.D., Biochemistry and Molecular Genetics University of Louisville 2011-2015

The Graduate Dean's Citation, University of Louisville - 2015

Biochemistry and Molecular Biology Retreat Oral Presentation - 2015

Society for Free Radical Biology and Medicine 
Oral Presentation - 2013

IPIBS Fellowship Ph.D. Program, Biochemistry and

Molecular Biology, University of Louisville - 2011

PROFESSIONAL

SOCIETIES: Society for Free Radical Biology and Medicine Member

PUBLICATIONS: $\quad$ Bonsignore, L.A., Tooley, J.G., Van Hoose, P. M., Wang, E., Cheng, A., Cole, M. P., and Schaner Tooley, C. E. (2015) NRMT1 knockout mice exhibit phenotypes associated with DNA repair and premature aging. Mechanisms of ageing and development 146-148, 42-52

Wysoczynski, M., Solanki, M., Borkowska, S., Van Hoose, P., Brittian, K. R., Prabhu, S. D., Ratajczak, M. Z., and Rokosh, G. (2014) Complement Component 3 is Necessary to Preserve Myocardium and Myocardial Function in Chronic Myocardial Infarction. Stem cells 32, 2502-2515

ABSTRACTS: $\quad$ Van Hoose, P.M., Piell, K.M., Kelm, N.Q., Cole, M.P. (2015) "Combination Treatment with Conjugated Linoleic Acid and Nitrite Attenuates Mitochondrial Dysfunction Following Myocardial Infarction" Free Radical Biology and Medicine.

Van Hoose, P. M., Piell, K.M., Kelm, N.Q., and Cole, M.P. (2014) "Conjugated Linoleic Acid and Nitrite Co-treatment alter Cardiac Mitochondrial Function and Complex Activity in Vivo". Free Radical Biology and Medicine 76: S18.

Van Hoose, P. M., Piell, K.M., Kelm, N.Q., and Cole, M.P. (2014) "Co-treatment of Conjugated Linoleic Acid and Nitrite alters Mitochondrial Function in vivo". Research! Louisville.

Van Hoose, P., Piell, K., and Cole, M. (2013) "PSS214-CoAdministration of Nitrite and Conjugated Linoleic Acid Promotes Changes in Cardiac Mitochondria in a Murine Model of Obesity." Free Radical Biology and Medicine 65: S98-99.

Van Hoose, P. M., Piell, K.M., and Cole, M.P. (2013) "Nitrite and Conjugated Linoleic Acid Treatment Promotes changes in Cardiac Mitochondrial Function and Activity in Murine Model of Obesity". Research! Louisville. 
NATIONAL

MEETING

PRESENTATIONS: Society of Free Radical Biology and Medicine- "Combination

Treatment with Conjugated Linoleic Acid and Nitrite Attenuates Mitochondrial Dysfunction Following Myocardial Infarction". 2015. Poster Presentation

Society of Free Radical Biology and Medicine- "Conjugated Linoleic Acid and Nitrite Co-treatment alter Cardiac

Mitochondrial Function and Complex Activity in Vivo". 2014. Poster Presentation

Society for Free Radical Biology and Medicine- "CoAdministration of Nitrite and Conjugated Linoleic Acid Promotes Changes in Cardiac Mitochondria in a Murine Model of Obesity." 2013. Oral Presentation. 